\title{
T-duality orbifolds of heterotic Narain compactifications
}

\author{
Stefan Groot Nibbelink ${ }^{a}$ and Patrick K.S. Vaudrevange ${ }^{b, c}$ \\ ${ }^{a}$ School of Engineering and Applied Sciences, Rotterdam University of Applied Sciences, \\ G.J. de Jonghweg 4-6, 3015 GG Rotterdam, Netherlands \\ ${ }^{b}$ Arnold Sommerfeld Center for Theoretical Physics, Ludwig-Maximilians-Universität München, \\ Theresienstraße 37, 80333 München, Germany \\ ${ }^{c}$ Physik Department T30, Technische Universität München, \\ James-Franck-Straße, 85748 Garching, Germany \\ E-mail: groos@hr.nl, patrick.vaudrevange@tum.de
}

ABSTRACT: To obtain a unified framework for symmetric and asymmetric heterotic orbifold constructions we provide a systematic study of Narain compactifications orbifolded by finite order $T$-duality subgroups. We review the generalized vielbein that parametrizes the Narain moduli space (i.e. the metric, the $B$-field and the Wilson lines) and introduce a convenient basis of generators of the heterotic $T$-duality group. Using this we generalize the space group description of orbifolds to Narain orbifolds. This yields a unified, crystallographic description of the orbifold twists, shifts as well as Narain moduli. In particular, we derive a character formula that counts the number of unfixed Narain moduli after orbifolding. Moreover, we develop new machinery that may ultimately open up the possibility for a full classification of Narain orbifolds. This is done by generalizing the geometrical concepts of $\mathbb{Q}^{-}, \mathbb{Z}^{-}$ and affine classes from the theory of crystallography to the Narain case. Finally, we give a variety of examples illustrating various aspects of Narain orbifolds, including novel $T$-folds.

Keywords: String Duality, Superstring Vacua, Superstrings and Heterotic Strings

ArXiv EPrint: 1703.05323 


\section{Contents}

1 Introduction and conclusions 1

2 Heterotic Narain torus compactifications 5

2.1 Worldsheet field content of the heterotic string 5

$\begin{array}{lll}2.2 & \text { Torus partition functions as Narain lattice sums } & 7\end{array}$

2.3 Narain lattices 8

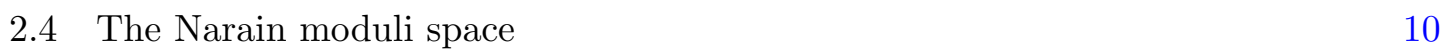

$\begin{array}{ll}2.5 & \text { Coordinate fields and momenta } \\ \end{array}$

3 The $\boldsymbol{T}$-duality group $\quad 14$

$\begin{array}{lll}3.1 & \text { Decomposition of the generalized vielbein } & 14\end{array}$

$\begin{array}{lll}3.2 & \text { Coset decomposition of the T-duality group } & 14\end{array}$

$\begin{array}{ll}3.3 & \text { Transformation of Narain moduli } \\ 3.4 & 17\end{array}$

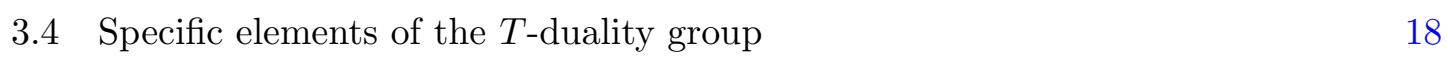

$\begin{array}{lll}3.4 .1 & \text { The geometric subgroup } & 18\end{array}$

$\begin{array}{lll}3.4 .2 & \text { Non-geometric elements } & 20\end{array}$

3.5 The maximal compact subgroup of $\mathrm{O}_{\widehat{\eta}}(D, D+16 ; \mathbb{R})$

4 Generalized space groups of Narain orbifolds $\quad 22$

4.1 Heterotic Narain orbifolds 22

$\begin{array}{lll}4.2 & \text { Generalized space group } & 23\end{array}$

4.3 Conditions on the twists $\Theta_{\alpha} \quad 24$

$\begin{array}{ll}4.4 \text { Orbifold projections of } \mathbb{\Gamma} & 25\end{array}$

4.5 Quantization of the generalized shifts $V_{\alpha} \quad 25$

4.6 Preserving at least $\mathcal{N}=1$ target-space supersymmetry 26

5 Moduli stabilization in Narain orbifolds $\quad 27$

$\begin{array}{ll}5.1 & \text { Narain orbifolds in the lattice basis } \\ 5.2\end{array}$

5.2 On the existence of Narain orbifolds for a given point group 29

5.3 Mapping from the lattice basis to the coordinate basis 30

5.4 Dimensionality of the Narain orbifold moduli space 31

5.5 A $T$-fold constructed as an asymmetric $\mathbb{Z}_{2}$ Narain orbifold 33

6 Towards a classification of Narain orbifolds $\quad 34$

6.1 Narain $\mathbb{Q}$ - and $\mathbb{Z}$-classes 34

6.2 Interpretation of Narain $\mathbb{Q}$ - and $\mathbb{Z}$-classes 34

6.3 Narain Poincaré-classes 36

6.4 Interpretation of Narain Poincaré-classes 36 
7 Symmetric orbifolds as Narain orbifolds

$\begin{array}{lll}7.1 & \text { Symmetric } \mathbb{Z}_{K} \text { orbifolds } & 38\end{array}$

$\begin{array}{lll}7.2 & \text { Moduli stabilization in symmetric } \mathbb{Z}_{K} \text { orbifolds } & 39\end{array}$

8 Two-dimensional Abelian Narain orbifolds $\quad 40$

$8.1(D, D)$-Narain orbifold formalism 41

8.2 Q- and $\mathbb{Z}$-classes of two-dimensional $\mathbb{Z}_{K}$ Narain orbifolds $\quad 42$

8.3 Two equivalent asymmetric $\mathbb{Z}_{12}$ Narain orbifolds 43

8.4 Exposing a seemingly asymmetric $\mathbb{Z}_{3}$ Narain orbifold 46

8.5 Symmetric $\mathbb{Z}_{2}$ Narain orbifolds from inequivalent $\mathbb{Z}$-classes 48

A Moduli deformations and the generalized metric $\quad \mathbf{5 0}$

A.1 T-duality transformations that leave a generalized metric invariant $\quad 50$

A.2 Infinitesimal moduli deformations of the Narain lattice 51

A.3 $\widehat{\mathrm{H}}$-invariant infinitesimal moduli deformations $\quad 51$

A.4 The number of $\widehat{H}$-invariant Narain moduli 52

\section{Introduction and conclusions}

Since the early days of superstring theory, the heterotic string [1-3] has served as a promising candidate theory for a unified quantum description of particle physics as well as gravity, see e.g. [4] for a textbook introduction to string phenomenology. One of the main obstacles lies in the fact that the heterotic string is conventionally defined in a ten-dimensional space-time. Hence, six spatial dimensions have to be compactified in order to make contact to the observable four-dimensional world.

One possibility is to compactify on a six-dimensional (symmetric) toroidal orbifold $[5,6]$ which is the quotient of a six-torus $T^{6}$ by some of its discrete isometries, see [7] for a full classification with $\mathcal{N} \geq 1$ supersymmetry in four dimensions. For example, one can use an Abelian rotational symmetry $\mathbb{Z}_{K}$ and define the orbifold geometrically as the quotient space $T^{6} / \mathbb{Z}_{K}$. Especially, in the presence of discrete Wilson lines [8] orbifold compactifications have been used to construct (minimal) supersymmetric extensions of the Standard Model (MSSM) from the heterotic string [9-26]. ${ }^{1}$ These constructions can be considered to be promising directions to connect string theory to particle physics: beside reproducing MSSM-like models, they offer an appealing geometrical interpretation, in which many properties of the elementary particles depend on their localization in extra dimensions [14, 3335]. Unfortunately, these constructions generically leave a number of moduli, like the compactification radius $R$, unfixed.

A possibility to stabilize moduli is to generalize the construction of symmetric orbifolds to asymmetric ones: in this case one quotients the compactification space not only

\footnotetext{
${ }^{1}$ For related MSSM model-building using compactifications of the heterotic string on Calabi-Yaus see e.g. [27-32].
} 
geometrically, but also by a genuine stringy symmetry [36]. The most famous example of such a symmetry of string theory is $T$-duality: in its simplest form, $T$-duality is a $\mathbb{Z}_{2}$ transformation that identifies a string compactification on a circle with small radius $R$ with another compactification on a circle with large radius $1 / R$. This is a full quantum duality on the string worldsheet as this can be described as field redefinitions in a path integral approach [37-39]. Now, in order to be able to perform the quotient by this $T$-duality transformation the radius $R$ can no longer be a free parameter, but it has to be fixed at the so-called self-dual value $R=1$ (in string units). This promotes the $T$-duality transformation $R \mapsto 1 / R$ to a symmetry of the theory. On the left- and right-moving coordinate fields $X_{1}$ and $X_{\mathrm{r}}$ this $T$-duality transformation is realized by $X_{1} \mapsto+X_{1}$ and $X_{\mathrm{r}} \mapsto-X_{\mathrm{r}}$. Hence, in general, such $T$-dualities act differently on the left- and right-moving degrees of freedom of the string and the resulting quotient spaces are often called asymmetric orbifolds [40]. Asymmetric orbifolds provide specific examples of non-geometric string backgrounds [4143] or so-called $T$-folds [44, 45]. More recently double field theory [46-48] was introduced as an attempt to obtain a setting with doubled geometry to describe such $T$-folds using geometrical tools inspired by a string field-theoretical description of the left- and right-moving string coordinates. Hence, asymmetric string constructions are of increasing interest in the connection to non-geometric flux backgrounds [49, 50]. Various aspects of asymmetric orbifolds have been studied in the past [51-59] and with recent renewed interest [60, 61] and in particular also in the context on non-supersymmetric constructions [62-64].

In contrast to symmetric orbifolds the phenomenological prospects of heterotic asymmetric orbifolds are far less studied. The main asymmetric activities in this direction concentrated up to now on the free fermionic construction of the heterotic string $[65,66]$. These free fermionic models naturally incorporate both, asymmetric as well as symmetric $\mathbb{Z}_{2}$ twists [67] and successful MSSM model-building has been carried out [68-71]. Furthermore, there has been some recent activities on model-building using asymmetric $\mathbb{Z}_{3}$ orbifolds [72-74]. Finally, asymmetric string constructions can be further generalized in the covariant lattice approach [75] which generalizes the Narain lattice [76], in phenomenologically promising Gepner models [77-81] and further with asymmetric CFTs [82-84].

Main results. In this work we develop a generalized space group description of Narain orbifolds and utilize this formalism throughout this work to study various aspects of symmetric and asymmetric orbifolds in a unified fashion. To define the generalized space group, we first perform a concise investigation of the heterotic $T$-duality group: we decompose its generators into geometrical and non-geometric ones and use them to parametrize the maximal compact subgroup of the $T$-duality group. This is important, as the maximal compact subgroup contains the finite subgroups that can be used to build (a)symmetric orbifolds. Hence, the generalized space group provides a unified framework to study symmetric and asymmetric orbifolds in a systematic manner.

We apply our understanding of the $T$-duality group to derive conditions for the stabilization of Narain moduli by orbifolding. This leads us to a closed character formula to count the number of unstabilized Narain moduli. In particular, this formula shows that all Narain moduli are fixed, if the left- and right-moving twists do not have any irreducible 
representations of the point group in common. We use our findings on moduli stabilization to formulate sufficient conditions for a Narain orbifold to exist crystallographically by reducing this question to the question whether certain Riccati equations admit solutions. Hence, using our generalized space group description one can check that a Narain orbifold exists at least crystallographically and one can identify the associated Narain torus that is compatible with the orbifold action.

Moreover, in this paper we lay the foundation for a classification of Narain orbifolds. Even though asymmetric orbifolds have been studied essentially since the birth of superstring theory, they have been analyzed so far essentially on a case-by-case basis. Based on our definition of the generalized space group we identify equivalence relations for Narain orbifolds. These equivalences extend the notations of $\mathbb{Q}_{-}, \mathbb{Z}$ - and affine-equivalences from theory of crystallography to the Narain case leading to the notions of Narain $\mathbb{Q}-, \mathbb{Z}$ - and Poincaré-classes. This can be seen as a first step towards a classification of symmetric as well as asymmetric Narain orbifolds, which includes - besides the information on the six-dimensional compactification space - also the anti-symmetric Kalb-Ramond $B$-field, the (discrete) Wilson lines and the orbifold shift-vectors in a unified fashion.

Finally, we construct a non-trivial set of (two-dimensional and more general) Narain orbifolds by specifying their generalized space groups. We use these examples to illustrate many aspects of our study, like the stabilization of Narain moduli and the equivalence classes for Narain orbifolds.

Outlook. In this work we investigated necessary conditions for a Narain orbifold to exist. However, we ignored possible extra conditions coming from modular invariance, as they have been studied in the past, see e.g. [53]. However, it would be advantageous to check for full modular invariance on the level of the generalized space group and, ultimately, to incorporate modular invariance in the definition of generalized space groups such that generalized space groups yield modular invariant Narain orbifolds by construction.

Moreover, we can imagine various applications of our work: the space group formulation of Narain orbifolds allows for a systematic construction of large sets of examples in various dimensions and in both, the $(D, D)$ case as well as the heterotic $(D, D+16)$ case. In addition, using our definitions of Narain $\mathbb{Q}-, \mathbb{Z}$ - and Poincaré classes one can unambiguously decide whether two Narain orbifold models are physically identical or not. This might proof to be very useful for systematic investigations and classifications for various reasons: first of all, in the traditional approach two (symmetric) orbifold models are often said to be equivalent if their massless matter spectra agree. However, this is neither necessary nor sufficient: for example, two different string constructions might possess identical massless spectra but different couplings, or the massless spectrum of a given toroidal orbifold compactification can be enhanced at specific points in its moduli space. Precisely here the Narain Poincaré classes would come to the rescue and decide for (in)equivalence. However, our new definition of equivalence might be computationally very intensive and, hence, further studies might be necessary in order to apply it practically for large computer scans.

Second, having an unambiguous criterion for two Narain orbifolds to be inequivalent, our work can be used to classify Narain orbifolds, both symmetric and asymmetric ones. 
Such a classification would automatically include the orbifold twists and shifts as well as the background fields, i.e. the torus metric, the $B$-field and (discrete) Wilson lines.

Finally, one can use our definitions of Narain $\mathbb{Q}$-, $\mathbb{Z}$ - and Poincaré classes to decide whether a Narain orbifold is genuine asymmetric or only seemingly. Hence, our approach might be also very helpful in the study of non-geometrical backgrounds for string theory in general, since it has been proven to be quite difficult to obtain concrete, yet true, examples of such backgrounds.

Paper outline. In section 2 we recall the basics of the Narain description of heterotic torus compactifications with continuous Wilson lines $A$, the anti-symmetric Kalb-Ramond $B$-field and the metric $G$. In this section we exploit the fact that the moduli space of Narain compactifications is concisely described as the coset of the continuous $T$-duality group over its maximal compact subgroup and the discrete $T$-duality group $\mathrm{O}_{\widehat{\eta}}(D, D+16 ; \mathbb{Z})$.

Given this prominent roles of continuous and discrete $T$-duality groups, we reserve section 3 to study their properties. In particular, we list a complete set of generators of $\mathrm{O}_{\widehat{\eta}}(D, D+16 ; \mathbb{R})$, which are chosen such that they parametrize the discrete $T$-duality group if their parameters are restricted to specific, quantized values. In addition, we give the nonlinear transformations of the moduli $G, B, A$ under arbitrary $T$-duality group elements.

After these preparations, section 4 sets up a generalized space group description of Narain orbifolds involving combined shift- and twist-elements. In this section various properties of Narain orbifolds are uncovered. In particular, we show that the shifts of the generalized space group are quantized in the directions in which the twists act trivially. Moreover, we emphasize that the amount of preserved target-space supersymmetry is solely decided by the twists $\theta_{\alpha r}$ that acts on the right-moving sector.

Section 5 investigates two related questions: i) under what conditions does a Narain orbifold exist and ii) how many Narain moduli, $G, B, A$, are fixed. To facilitate this discussion the lattice basis is introduced in which the twists are represented by integral matrices $\widehat{\rho}_{\alpha} \in \mathrm{O}_{\widehat{\eta}}(D, D+16 ; \mathbb{Z})$. Some properties of these twists in the lattice basis can concisely be characterized using the generalized metric $\mathcal{H}$ and the associated $\mathbb{Z}_{2}$-grading $\mathcal{Z}$. By exploiting the coset structure of the Narain moduli space, we show that a Narain orbifold exists provided that certain Ricatti equations, i.e. coupled matrix equations, have a solution. Deformations of such a solution correspond to the unconstrained moduli of a Narain orbifold. Using some results collected in appendix A we derive a character formula to count their number.

All these results are used in section 6 to lay the foundations for a classification of Narain orbifolds. Given that the concepts of $\mathbb{Q}^{-}, \mathbb{Z}$ - and affine-classes proved to be very useful for the classification of symmetric orbifolds, we extend these concepts to Narain orbifolds.

To illustrate the power of the generalized space group description of Narain orbifolds we study symmetric orbifolds in section 7 in this language. Even though the main interest of Narain orbifolds lies in the construction of asymmetric orbifolds (or $T$-folds), we show in this section that the language of Narain orbifolds gives a convenient, unified description of the geometry and the (discrete) Wilson lines. 
Finally, in section 8 we employ the Narain $\mathbb{Q}$ - and $\mathbb{Z}$-classes to study two-dimensional Abelian $\mathbb{Z}_{K}$ Narain orbifolds. We provide a large table with many examples of previously unknown two-dimensional Narain orbifolds. By an explicit construction we show that it is possible to have a $\mathbb{Z}_{12}$ two-dimensional Narain orbifold, while it is well-known that the largest order of Euclidean $\mathbb{Z}_{K}$ twists is $K=6$ in two dimensions. Moreover, $\mathbb{Q}$ - and $\mathbb{Z}$ classes are particularly useful to distinguish seemingly asymmetric from truly asymmetric orbifolds as we illustrate by various examples.

\section{Heterotic Narain torus compactifications}

This section reviews the Narain formulation of heterotic torus compactifications [76] and sets the notation used throughout this work. The moduli space can be described using the generalized vielbein $E$, which is parametrized by continuous Wilson lines $A$, the antisymmetric Kalb-Ramond $B$-field and the metric $G$. This vielbein characterizes coordinate field boundary conditions as well as the momenta that appear in the representation of the Narain torus partition function as a lattice sum.

\subsection{Worldsheet field content of the heterotic string}

We parametrize the two-dimensional string worldsheet by (real) coordinates $\sigma$ and $\bar{\sigma}$, defined by

$$
\sigma=\sigma_{1}+\sigma_{0}, \quad \bar{\sigma}=\sigma_{1}-\sigma_{0}
$$

where $\sigma_{0}$ and $\sigma_{1}$ denote the worldsheet time and space coordinate, respectively. Worldsheet fields that solely depend on $\sigma$ or $\bar{\sigma}$ are called left-moving or right-moving fields, respectively. They are correspondingly labelled by a subscript 1 or $\mathrm{r}$ (or in capital letters $\mathrm{L} / \mathrm{R}$ ). The heterotic string is closed because of the identification $\left(\sigma_{0}, \sigma_{1}\right) \sim\left(\sigma_{0}, \sigma_{1}+1\right)$. Hence, $\left(\sigma_{0}, \sigma_{1}\right)$ are coordinates on a worldsheet cylinder for the freely propagating string.

The heterotic string [1-3] is described by a conformal field theory on the worldsheet with 26 left-moving real bosonic fields and ten right-moving real bosonic and fermionic fields.

The easiest approach to connect this theory to particle physics in $d$ dimensions (for example $d=4$ ) is to perform a stepwise compactification: in the first step one compactifies the 16 surplus left-moving bosonic fields on a 16-dimensional torus in order to match the number of left- and right-moving bosonic fields to ten. The resulting theory corresponds to a ten-dimensional theory with a gauge group dictated by modular invariance of the string partition function. For example, in the case of ten-dimensional $\mathcal{N}=1$ supersymmetry the gauge group is fixed to either $\mathrm{E}_{8} \times \mathrm{E}_{8}$ or $\mathrm{SO}(32)$. Then, in a second step one compactifies on a $D$-dimensional space, for example on a Calabi-Yau or an orbifold. As a result one obtains a $d$-dimensional theory, where $d+D=10$, e.g. $4+6=10$. An alternative approach, which we use in this paper, is the so-called Narain construction, where the twostep compactification described above is performed in a single step compactification of the heterotic string directly to $d$ dimensions, see section 2.2 . 
In light-cone gauge two left- and right-moving uncompactified dimensions are gaugefixed and, hence, eliminated. Thus, the heterotic string in light-cone gauge can be described by the following worldsheet fields:

- As left-moving fields, there are $8+16=24$ real bosonic fields. They are denoted by $x_{1}^{\mu}(\sigma)$ with $\mu=2, \ldots, d-1$ ( $\mu=0,1$ are chosen to be fixed in light-cone gauge) for the uncompactified and $Y_{\mathrm{L}}(\sigma)$ for the compactified dimensions, respectively. Furthermore, we set

$$
Y_{\mathrm{L}}(\sigma)=\left(\begin{array}{c}
y_{1}(\sigma) \\
y_{\mathrm{L}}(\sigma)
\end{array}\right)
$$

where $y_{1}(\sigma)=\left(y_{1}^{i}(\sigma)\right)$ for $i=1, \ldots, D$ live on the $D$-dimensional compactification space. In addition, $y_{\mathrm{L}}(\sigma)=\left(y_{\mathrm{L}}^{I}(\sigma)\right)$ for $I=1, \ldots, 16$ are often referred to as the gauge degrees of freedom.

- As right-moving fields, there are eight real bosonic fields plus their real fermionic superpartners. They are denoted by $\left(x_{\mathrm{r}}^{\mu}(\bar{\sigma}), y_{\mathrm{r}}^{i}(\bar{\sigma})\right)$ and $\psi_{\mathrm{R}}(\bar{\sigma})=\left(\psi_{\mathrm{R}}^{\mu}(\bar{\sigma}), \psi_{\mathrm{R}}^{i}(\bar{\sigma})\right)$, respectively, with $\mu=2, \ldots, d-1$ and $i=1, \ldots, D$.

Left- and right-moving bosonic fields can be combined to coordinate fields $x^{\mu}(\sigma, \bar{\sigma})$ and $X^{i}(\sigma, \bar{\sigma})$ which parametrize the $d$ uncompactified and $D$ compactified dimensions, respectively, i.e.

$$
x^{\mu}(\sigma, \bar{\sigma})=\frac{1}{\sqrt{2}}\left(x_{\mathrm{r}}^{\mu}(\bar{\sigma})+x_{1}^{\mu}(\sigma)\right) \quad \text { and } \quad X^{i}(\sigma, \bar{\sigma})=\frac{1}{\sqrt{2}}\left(y_{\mathrm{r}}^{i}(\bar{\sigma})+y_{1}^{i}(\sigma)\right) .
$$

Their classical equations of motion read

$$
\partial_{\sigma} \partial_{\bar{\sigma}} x^{\mu}(\sigma, \bar{\sigma})=0 \quad \text { and } \quad \partial_{\sigma} \partial_{\bar{\sigma}} X^{i}(\sigma, \bar{\sigma})=0,
$$

which is solved by the general ansatz (2.3).

Hence, collectively, we have $2 D+16$ compactified bosonic worldsheet fields $Y$ nested in the following fashions:

$$
Y(\sigma, \bar{\sigma})=\left(\begin{array}{c}
y_{\mathrm{r}}(\bar{\sigma}) \\
y_{1}(\sigma) \\
y_{\mathrm{L}}(\sigma)
\end{array}\right), \quad y(\sigma, \bar{\sigma})=\left(\begin{array}{c}
y_{\mathrm{r}}(\bar{\sigma}) \\
y_{1}(\sigma)
\end{array}\right), \quad Y_{\mathrm{L}}(\sigma)=\left(\begin{array}{c}
y_{1}(\sigma) \\
y_{\mathrm{L}}(\sigma)
\end{array}\right) .
$$

We define the following dimensions: $D_{\mathrm{r}}=D_{\mathrm{l}}=D$ and $D_{\mathrm{L}}=D_{\mathrm{l}}+16=D+16$. We will use the same notation as in eq. (2.5) for other types of vectors.

The separation (2.3) of the coordinate fields $X^{i}(\sigma, \bar{\sigma})$ into left- and right-moving coordinates $y_{1}^{i}(\sigma)$ and $y_{\mathrm{r}}^{i}(\bar{\sigma})$ is unique up to a constant shift of the zero modes $\xi^{i}$, i.e.

$Y(\sigma, \bar{\sigma}) \sim Y(\sigma, \bar{\sigma})+\Xi, \quad \Xi=(\xi,-\xi, 0): \quad y_{\mathrm{r}}^{i}(\bar{\sigma}) \sim y_{\mathrm{r}}^{i}(\bar{\sigma})+\xi^{i}, \quad y_{1}^{i}(\sigma) \sim y_{1}^{i}(\sigma)-\xi^{i}$,

with $\xi \in \mathbb{R}^{D}$. This has important consequences for the number of worldsheet degrees of freedom: if one counts left- and right-movers $y(\sigma, \bar{\sigma}) \in \mathbb{R}^{2 D}$ independently there seems to be a doubling of degrees of freedom on the worldsheet compared to the coordinate fields $X(\sigma, \bar{\sigma}) \in \mathbb{R}^{D}$, see eq. (2.3). However, due to eq. (2.6) there are only $D$ independent zero-modes of $y(\sigma, \bar{\sigma})$ that specify the position of the string and the numbers of worldsheet degrees of freedom are equal for $X(\sigma, \bar{\sigma})$ and $y(\sigma, \bar{\sigma})$. 


\subsection{Torus partition functions as Narain lattice sums}

We consider torus compactifications $T_{\mathbb{\Gamma}}^{2 D+16}=\mathbb{R}^{2 D+16} / \mathbb{\Gamma}$ of the $2 D+16$ bosonic worldsheet fields $Y . \mathbb{\Gamma}$ is a so-called $2 D+16$-dimensional Narain lattice, which we will analyze in this section in detail. This will be of use when we discuss the more general case of Narain orbifolds later in section 4.

In the case of a Narain torus, the closed string boundary conditions of the worldsheet fields are given by

$$
x(\sigma+1, \bar{\sigma}+1)=x(\sigma, \bar{\sigma}), \quad \psi_{\mathrm{R}}(\bar{\sigma}+1)=(-)^{s} \psi_{\mathrm{R}}(\bar{\sigma}), \quad Y(\sigma+1, \bar{\sigma}+1)=Y(\sigma, \bar{\sigma})+L,
$$

where $s=0,1$ parametrizes the different spin structures of the right-moving fermions $\psi_{\text {R }}$, i.e. $s=0$ yields the so-called Ramond sector and $s=1$ the Neveu-Schwarz sector. Furthermore, $L \in \mathbb{\Gamma}$ denotes a lattice vector of $\mathbb{\Gamma}$.

At one-loop the partition function $Z_{\text {full }}(\tau, \bar{\tau})$ is given by the string vacuum-to-vacuum amplitude which corresponds to a worldsheet torus. This torus is defined by two periodicities of worldsheet fields: $\left(\sigma_{0}, \sigma_{1}\right) \sim\left(\sigma_{0}, \sigma_{1}+1\right)$ and $\left(\sigma_{0}, \sigma_{1}\right) \sim\left(\sigma_{0}+\tau_{2}, \sigma_{1}+\tau_{1}\right)$ for the string to close in the worldsheet-spatial and worldsheet-time directions, respectively. Here, $\tau=\tau_{1}+i \tau_{2}$ is the so-called modular parameter of the torus. Then, the full partition function $Z_{\text {full }}(\tau, \bar{\tau})$ of the one-loop worldsheet torus can be factorized as follows

$$
Z_{\text {full }}(\tau, \bar{\tau})=Z_{x}(\tau, \bar{\tau}) Z_{\psi}(\bar{\tau}) Z_{Y}(\tau, \bar{\tau}) .
$$

The individual partition functions are given by

$$
\begin{aligned}
& Z_{x}(\tau, \bar{\tau})=\frac{1}{\tau_{2}^{d / 2-1}}\left|\frac{1}{\eta^{(d-2)}(\tau)}\right|^{2}, \\
& Z_{\psi}(\bar{\tau})=\frac{1}{2} \frac{1}{\bar{\eta}^{4}(\bar{\tau})} \sum_{s, s^{\prime}=0}^{1} e^{\pi i\left(s+s^{\prime}+s^{\prime} s\right)} \bar{\theta}\left[\begin{array}{c}
\frac{s}{2} e_{4} \\
\frac{s^{\prime}}{2} e_{4}
\end{array}\right],
\end{aligned}
$$

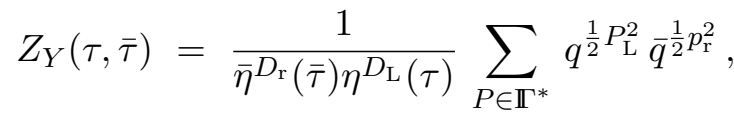

where $q=e^{2 \pi i \tau}, \bar{q}=e^{-2 \pi i \bar{\tau}}$ and $e_{d}=(1, \ldots, 1)$ denotes the $d$-dimensional vector with all entries equal to one. Here and in the following we often omit the dependencies on $\tau$ and $\bar{\tau}$ for notational ease. In addition, $\eta(\tau)$ denotes the Dedekind function and $\theta$ the theta-function. The vectors $P$ are from the dual lattice $\mathbb{\Gamma}^{*}$ which is defined as $P \in \mathbb{\Gamma}^{*}$ if

$$
P^{T} \eta L \in \mathbb{Z},
$$

for any $L \in \mathbb{\Gamma}$. Here, we have introduced the Lorentzian inner product of lattice vectors as

$$
P^{T} \eta P^{\prime}=-p_{\mathrm{r}}^{T} p_{\mathrm{r}}^{\prime}+P_{\mathrm{L}}^{T} P_{\mathrm{L}}^{\prime}, \quad \text { using } \quad P=\left(\begin{array}{c}
p_{\mathrm{r}} \\
P_{\mathrm{L}}
\end{array}\right) \quad \text { and } \quad \eta=\left(\begin{array}{cc}
-\mathbb{1}_{D} & 0 \\
0 & \mathbb{1}_{16+D}
\end{array}\right) \text {. }
$$

The metric $\eta$ should not be confused with the Dedekind function $\eta(\tau)$ that appears in partition functions; we assume that the reader understands from the context which is meant. 
The partition function $Z_{\psi}$ for the right-moving fermions can also be presented as a lattice sum, i.e. from $(2.9 \mathrm{~b})$ we get

$$
Z_{\psi}(\bar{\tau})=\frac{1}{\bar{\eta}^{4}(\bar{\tau})} \sum_{p_{\mathrm{R}} \in \Gamma_{\psi}} \bar{q}^{\frac{1}{2} p_{\mathrm{R}}^{2}}(-1)^{F},
$$

where the lattice $\Gamma_{\psi}=\Gamma_{\text {vec }} \oplus \Gamma_{\text {spin }}$ consists of the vectorial and spinorial weight lattices, given by $\Gamma_{\text {vec }}=\left\{p_{\mathrm{R}} \in \mathbb{Z}^{4} \mid p_{\mathrm{R}}^{T} e_{4}=\right.$ odd $\}$ and $\Gamma_{\text {spin }}=\left\{p_{\mathrm{R}}+\frac{1}{2} e_{4} \mid p_{\mathrm{R}} \in \mathbb{Z}^{4}\right.$ and $p_{\mathrm{R}}^{T} e_{4}=$ even $\}$. Furthermore, $F$ is the target-space fermion number, i.e. $F=0$ for $p_{\mathrm{R}} \in \Gamma_{\text {vec }}$ and $F=1$ for $p_{\mathrm{R}} \in \Gamma_{\text {spin }}$.

Eq. (2.12) can also be obtained as follows: the eight real worldsheet fermions $\psi_{\mathrm{R}}=\left(\psi_{\mathrm{R}}^{\mu}, \psi_{\mathrm{R}}^{i}\right)$ can be grouped in four complex fermions $\psi_{\mathrm{R}}=\left(\psi_{\mathrm{R}}^{m}, \psi_{\mathrm{R}}^{a}\right)$, where $m=$ $1, \ldots, d / 2-1$ and $a=1, \ldots, D / 2$ correspond to the uncompactified and compactified dimensions, respectively. Then, one can bosonize the complex fermions. Consequently, the bosonized fermions carry momentum $p_{\mathrm{R}}=\left(p_{\mathrm{R}}^{m}, p_{\mathrm{R}}^{a}\right)$ and the associated partition function coincides with eq. (2.12). The momentum $p_{R}^{m}$ has an important target-space interpretation: a string state with $p_{\mathrm{R}}^{m}$ being integer or half-integer signals a target-space boson or fermion in $d$ dimensions, respectively.

Modular invariance. The full partition function is required to be modular invariant: at one-loop the worldsheet has the topology of a torus with modular parameter $\tau$. Not all $\tau \in \mathbb{C}$ with $\operatorname{Im}(\tau)>0$ parametrize inequivalent worldsheet tori. Because of conformal symmetry tori related by the modular transformations

$$
T: \tau \rightarrow \tau+1, \quad S: \tau \rightarrow-1 / \tau,
$$

give the same physics. $T$ and $S$ generate the modular group $\operatorname{PSL}(2, \mathbb{Z})$. Invariance of the partition function (2.8) under $T$ and $S$ transformations requires that

$$
\forall P \in \mathbb{\Gamma}: \quad \frac{1}{2} P^{T} \eta P \equiv 0 \quad \text { and } \quad \mathbb{\Gamma}^{*}=\mathbb{\Gamma},
$$

where $a \equiv b$ means that $a$ and $b$ are equal up to some integer. These conditions tell us that $\mathbb{\Gamma}$ is an even self-dual lattice with signature $(D, D+16)$; the so-called Narain lattice. Note that vectors $P \in \mathbb{\Gamma}$ can be redefined as

$$
P \rightarrow U P
$$

for $U \in \mathrm{O}(D ; \mathbb{R}) \times \mathrm{O}(D+16 ; \mathbb{R})$ without changing the partition function (2.8).

\subsection{Narain lattices}

We analyse the conditions (2.14) in more detail. To do so, we may parametrize a general lattice vector $P \in \mathbb{\Gamma}$ as

$$
P=E N, \quad N=\left(\begin{array}{c}
m \\
n \\
q
\end{array}\right) \in \mathbb{Z}^{2 D+16},
$$


in terms of an invertible matrix $E$. This matrix $E$ is called the generalized vielbein of the Narain lattice $\mathbb{\Gamma}$ as its columns correspond to $2 D+16$ basis vectors of the lattice $\mathbb{\Gamma}$. The components of the vector $N$ can be interpreted as winding numbers $m \in \mathbb{Z}^{D}$, Kaluza-Klein numbers $n \in \mathbb{Z}^{D}$ and gauge lattice numbers $q \in \mathbb{Z}^{16}$. From the vielbein $E$ we can define the Narain metric $\widehat{\eta}$ as

$$
\widehat{\eta}=E^{T} \eta E
$$

Then, the scalar product of two vectors $P_{i}=E N_{i} \in \mathbb{\Gamma}$ for $i=1,2$ is given by

$$
P_{1}^{T} \eta P_{2}=N_{1}^{T}\left(E^{T} \eta E\right) N_{2}=N_{1}^{T} \widehat{\eta} N_{2}
$$

Hence, the lattice $\mathbb{\Gamma}$ is even if

$$
P^{T} \eta P=N^{T} \widehat{\eta} N \in 2 \mathbb{Z} .
$$

Note that an even lattice is automatically integral, i.e. $P^{T} \eta P^{\prime}=N^{T} \widehat{\eta} N^{\prime} \in \mathbb{Z}$. Therefore, the Narain metric $\widehat{\eta}$ is a symmetric, integer matrix with even entries on the diagonal and signature $(D, D+16)$. The dual lattice $\mathbb{\Gamma}^{*}$ is spanned by the dual vielbein $E^{*}$ which is defined as

$$
\left(E^{*}\right)^{T} \eta E=\mathbb{1}_{2 D+16},
$$

so that for a given $P=E^{*} N \in \mathbb{\Gamma}^{*}$ we have $P^{T} \eta P^{\prime} \equiv 0$ for all $P^{\prime}=E N^{\prime} \in \mathbb{\Gamma}$. By comparing this equation with (2.17) one infers that the dual basis is given by

$$
E^{*}=E \widehat{\eta}^{-1}
$$

Two lattices are identical if their vielbeins are related by a $\mathrm{GL}(2 D+16 ; \mathbb{Z})$ transformation. Hence, $\mathbb{\Gamma}$ is self-dual, $\mathbb{\Gamma}^{*}=\mathbb{\Gamma}$, if the Narain metric in eq. (2.21) satisfies

$$
\widehat{\eta} \in \mathrm{GL}(2 D+16 ; \mathbb{Z})
$$

Consequently, $\operatorname{det} \widehat{\eta}= \pm 1$ and we see from eq. (2.17) that the volume of the unit cell spanned by the vielbein $E$ is given by $\operatorname{vol}(\mathbb{\Gamma})= \pm \operatorname{det} E=1$.

It is often convenient to choose a special representation of the Narain metric. If not stated otherwise we will use

$$
\widehat{\eta}=\left(\begin{array}{ccc}
0 & \mathbb{1}_{D} & 0 \\
\mathbb{1}_{D} & 0 & 0 \\
0 & 0 & g
\end{array}\right),
$$

where $g$ is the metric of an even, self-dual 16-dimensional lattice. (Throughout this paper we use a hatted notation to refer to objects that are naturally defined in the lattice basis.) We choose it to be the Cartan matrix of $\mathrm{E}_{8} \times \mathrm{E}_{8}$ and write $g=\alpha_{\mathrm{g}}^{T} \alpha_{\mathrm{g}}$ where the columns of $\alpha_{\mathrm{g}}$ are the 16 simple root vectors of $\mathrm{E}_{8} \times \mathrm{E}_{8}$. The explicit expression for $\alpha_{\mathrm{g}}$ is given by

$$
\alpha_{\mathrm{g}}=\left(\begin{array}{cc}
\alpha\left(\mathrm{E}_{8}\right) & 0 \\
0 & \alpha\left(\mathrm{E}_{8}\right)
\end{array}\right)
$$


The columns of $\alpha\left(\mathrm{E}_{8}\right)$ represent the eight simple roots $\alpha^{I}\left(\mathrm{E}_{8}\right), I=1, \ldots, 8$, of the exceptional Lie algebra $\mathrm{E}_{8}$. They can be chosen as follows

$$
\alpha\left(\mathrm{E}_{8}\right)=\left(\begin{array}{cccccccc}
1 & 0 & 0 & 0 & 0 & 0 & 0 & -\frac{1}{2} \\
-1 & 1 & 0 & 0 & 0 & 0 & 0 & -\frac{1}{2} \\
0 & -1 & 1 & 0 & 0 & 0 & 0 & -\frac{1}{2} \\
0 & 0 & -1 & 1 & 0 & 0 & 0 & -\frac{1}{2} \\
0 & 0 & 0 & -1 & 1 & 0 & 0 & -\frac{1}{2} \\
0 & 0 & 0 & 0 & -1 & 1 & 1 & -\frac{1}{2} \\
0 & 0 & 0 & 0 & 0 & -1 & 1 & -\frac{1}{2} \\
0 & 0 & 0 & 0 & 0 & 0 & 0 & -\frac{1}{2}
\end{array}\right) .
$$

\subsection{The Narain moduli space}

Given the choice of a Narain metric $\widehat{\eta}$ in eq. (2.23) it is natural to look for a corresponding generalized vielbein $E$, which yields this Narain metric $E^{T} \eta E=\widehat{\eta}$. We see that a particular solution $R$ to equation (2.17) is given by

$$
R=\left(\begin{array}{ccc}
\frac{1}{\sqrt{2}} \mathbb{1}_{D} & \frac{-1}{\sqrt{2}} \mathbb{1}_{D} & 0 \\
\frac{1}{\sqrt{2}} \mathbb{1}_{D} & \frac{1}{\sqrt{2}} \mathbb{1}_{D} & 0 \\
0 & 0 & \alpha_{\mathrm{g}}
\end{array}\right) \quad \text { with } \quad R^{T} \eta R=\widehat{\eta}
$$

The general solution to (2.17) can be written in terms of this particular solution as

$$
E=U R \widehat{E}
$$

so that consequently,

$$
E^{T} \eta E=\widehat{E}^{T} \widehat{\eta} \widehat{E}=\widehat{\eta},
$$

if $U \in \mathrm{O}_{\eta}(D, D+16 ; \mathbb{R})$ and $\widehat{E} \in \mathrm{O}_{\widehat{\eta}}(D, D+16 ; \mathbb{R})$, i.e. if $U^{T} \eta U=\eta$ and $\widehat{E}^{T} \widehat{\eta} \widehat{E}=\widehat{\eta}$.

In the following we want to identify which transformations $U$ and $\widehat{E}$ in eq. (2.27) map between physically inequivalent theories and which do not. Therefore, we will identify the moduli space of heterotic Narain constructions. To do so, we define ${ }^{2}$

$$
\widehat{U}=R^{-1} U R
$$

and note that $\widehat{U} \in \mathrm{O}_{\widehat{\eta}}(D, D+16 ; \mathbb{R})$ if $U \in \mathrm{O}_{\eta}(D, D+16 ; \mathbb{R})$. Now, take a general vielbein $E=U R \widehat{E}$. Then, one can absorb $U$ into a redefinition of $\widehat{E}$ by defining $\widehat{E}^{\prime}$ as

$$
\widehat{E}^{\prime}=\widehat{U} \widehat{E}=\left(R^{-1} U R\right) \widehat{E} \in \mathrm{O}_{\widehat{\eta}}(D, D+16 ; \mathbb{R}) \text { hence } E=R \widehat{E}^{\prime} .
$$

However, it is not useful to absorb all $U$ transformations in eq. (2.27) into a redefinition of $\widehat{E}$ : consider $U \in \mathrm{O}(D ; \mathbb{R}) \times \mathrm{O}(D+16 ; \mathbb{R}) \subset \mathrm{O}_{\eta}(D, D+16)$. As the partition function (2.8) depends only on $P_{\mathrm{L}}^{2}$ and $p_{\mathrm{r}}^{2}$ such transformations leave the partition function invariant. Thus, $U \in \mathrm{O}(D ; \mathbb{R}) \times \mathrm{O}(D+16 ; \mathbb{R})$ in eq. (2.27) maps physically equivalent theories to

\footnotetext{
${ }^{2}$ In the remainder of this paper we will use this conjugation with $R$ to switch between $\mathrm{O}_{\eta}$ and $\mathrm{O}_{\widehat{\eta}}$ group elements.
} 
each other. On the other hand, $\widehat{E}$ transformations in eq. (2.27) change the partition function (2.8) in general. Therefore, $\widehat{E}$ contains the parameters (i.e. the moduli) that continuously deform the Narain lattice with vielbein $R$ to Narain lattices with vielbeins $R \widehat{E}$, which are in general physically inequivalent but share the same Narain metric $\widehat{\eta}$. However, not all vielbeins $\widehat{E}$ are physically inequivalent: consider two vielbeins $E, E^{\prime}$ for two Narain lattices $\mathbb{\Gamma}, \mathbb{\Gamma}^{\prime}$ satisfying (2.17). Under what condition(s) do these backgrounds describe the same Narain lattice $\mathbb{\Gamma}^{\prime}=\mathbb{\Gamma}$ ? This happens when for each point $P \in \mathbb{\Gamma}$ there is a unique point $P^{\prime} \in \mathbb{\Gamma}^{\prime}$ which is identical to it: in the parametrization (2.16) this amounts to

$$
U E N=U P=P^{\prime}=E^{\prime} N^{\prime}
$$

such that the integer vectors $N$ and $N^{\prime}$ are mapped to each other one-to-one, i.e. $N=\widehat{M} N^{\prime}$ with $\widehat{M}=\left(E^{-1} U^{T} E^{\prime}\right)$. Note that we added in eq. (2.31) a rotation matrix $U \in \mathrm{O}(D ; \mathbb{R}) \times \mathrm{O}(D+16 ; \mathbb{R})$, which is unphysical as discussed above. Hence, the Narain lattices $\mathbb{\Gamma}$ and $\mathbb{\Gamma}^{\prime}$ are the same if there exists a rotation matrix $U$ such that $\widehat{M} \in \mathrm{GL}(2 D+16 ; \mathbb{Z})$. Moreover, we assumed that both $E$ and $E^{\prime}$ give the same Narain metric $\widehat{\eta}$, see (2.17). This implies that the matrix $\widehat{M}$ is actually an element of the so-called $T$-duality group $\mathrm{O}_{\widehat{\eta}}(D, D+16 ; \mathbb{Z})$, i.e.

$$
\widehat{M}^{T} \widehat{\eta} \widehat{M}=\widehat{\eta}
$$

(More details on the $T$-duality group $\mathrm{O}_{\widehat{\eta}}(D, D+16 ; \mathbb{Z})$ are given in section 3.) Therefore, Narain compactifications based on the vielbeins $E=R \widehat{E}$ and $E^{\prime}=U E \widehat{M}$ are physically equivalent, i.e.

$$
E=R \widehat{E} \sim E^{\prime}=U R \widehat{E} \widehat{M},
$$

if $U \in \mathrm{O}(D ; \mathbb{R}) \times \mathrm{O}(D+16 ; \mathbb{R})$ and $\widehat{M} \in \mathrm{O}_{\widehat{\eta}}(D, D+16 ; \mathbb{Z})$. In terms of $\widehat{E}$ this equivalence relation reads

$$
\widehat{E} \sim \widehat{U} \widehat{E} \widehat{M}
$$

where $\widehat{U}=R^{-1} U R$. This equivalence relation can be used to define a quotient space. Consequently, the moduli space of Narain compactifications is uniquely parametrized by an element $\widehat{E}$ in the coset

$$
\mathcal{M}=\mathrm{O}(D ; \mathbb{R}) \times \mathrm{O}(D+16 ; \mathbb{R}) \backslash \mathrm{O}_{\widehat{\eta}}(D, D+16 ; \mathbb{R}) / \mathrm{O}_{\widehat{\eta}}(D, D+16 ; \mathbb{Z}) .
$$

Here, it is understood that the first two factors in the denominator act from the left (via $\widehat{U}$ ) while the last factor acts from the right (via $\widehat{M}$ ), see eq. (2.34). The $T$-duality transformations $\widehat{M}$ are said to change the duality frame.

An explicit parametrization of the matrix $U \in \mathrm{O}(D ; \mathbb{R}) \times \mathrm{O}(D+16 ; \mathbb{R})$, satisfying

$$
U^{T} U=\mathbb{1}, \quad U^{T} \eta U=\eta
$$

is given by

$$
U=\left(\begin{array}{ccc}
u_{\mathrm{r}} & 0 & 0 \\
0 & u_{\mathrm{l}} & u_{\mathrm{lL}} \\
0 & u_{\mathrm{Ll}} & u_{\mathrm{L}}
\end{array}\right)
$$


provided that the constraints $u_{\mathrm{r}}^{T} u_{\mathrm{r}}=u_{1}^{T} u_{1}+u_{\mathrm{Ll}}^{T} u_{\mathrm{Ll}}=\mathbb{1}_{D}, u_{\mathrm{lL}}^{T} u_{\mathrm{lL}}+u_{\mathrm{L}}^{T} u_{\mathrm{L}}=\mathbb{1}_{16}$ and $u_{1}^{T} u_{\mathrm{lL}}+u_{\mathrm{Ll}}^{T} u_{\mathrm{L}}=0$ are fulfilled. As we have already seen above, often the closely related matrix

$$
\widehat{U}=R^{-1} U R=\left(\begin{array}{ccc}
u_{+} & u_{-} & \frac{1}{\sqrt{2}} u_{\mathrm{lL}} \alpha_{\mathrm{g}} \\
u_{-} & u_{+} & \frac{1}{\sqrt{2}} u_{\mathrm{lL}} \alpha_{\mathrm{g}} \\
\frac{1}{\sqrt{2}} \alpha_{\mathrm{g}}^{-1} u_{\mathrm{Ll}} & \frac{1}{\sqrt{2}} \alpha_{\mathrm{g}}^{-1} u_{\mathrm{Ll}} & \alpha_{\mathrm{g}}^{-1} u_{\mathrm{L}} \alpha_{\mathrm{g}}
\end{array}\right), \quad \text { where } \quad u_{ \pm}=\frac{1}{2}\left(u_{\mathrm{l}} \pm u_{\mathrm{r}}\right)
$$

is more convenient.

Modulo the transformations $\widehat{U}$ and $\widehat{M}$, the general solution to eq. (2.28) can be represented as

$$
\widehat{E}=\left(\begin{array}{ccc}
e & 0 & 0 \\
-e^{-T} C^{T} & e^{-T} & -e^{-T} A^{T} \alpha_{\mathrm{g}} \\
\alpha_{\mathrm{g}}^{-1} A & 0 & \mathbb{1}_{16}
\end{array}\right), \quad C=B+\frac{1}{2} A^{T} A
$$

Hence, $\widehat{E}=\widehat{E}(e, B, A)$ is parametrized by the Narain moduli $e, B$ and $A$, where $e$ is the $D$-dimensional vielbein of the $D$-torus with metric $G=e^{T} e . A$ is a $16 \times D$ matrix, whose $i$-th column contains the Wilson line which is associated to the $i$-th basis vector in $e$ and, finally, $B$ denotes the anti-symmetric Kalb-Ramond $B$-field.

In summary, we can specify the most general form of the generalized vielbein $E$ with Narain metric $\widehat{\eta}=E^{T} \eta E$ as given in eq. (2.23). It reads

$$
E=U R \widehat{E} \widehat{M}
$$

with $U \in \mathrm{O}(D ; \mathbb{R}) \times \mathrm{O}(D+16 ; \mathbb{R})$ and $\widehat{M} \in \mathrm{O}_{\widehat{\eta}}(D, D+16 ; \mathbb{Z})$. The matrix $R$ is given in eq. (2.26) and the moduli dependent part $\widehat{E}=\widehat{E}(e, B, A)$ is specified in eq. (2.39). In fact, we may take $\widehat{M}=\mathbb{1}$ without loss of generality as we show in section 3.2 .

Equivalent Narain metrics. One may encounter different Narain metrics, say $\widehat{\eta}$ and $\widehat{\eta}^{\prime}$ from $\mathrm{GL}(2 D+16 ; \mathbb{Z})$, such that

$$
E^{T} \eta E=\widehat{\eta}, \quad E^{\prime T} \eta E^{\prime}=\widehat{\eta}^{\prime} .
$$

In this case one cannot immediately compare the moduli in $E$ and $E^{\prime}$, because their hatted versions $\widehat{E}$ and $\widehat{E}^{\prime}$ lie in two different moduli spaces. Since we are talking about two representations of the same Narain lattice we have

$$
E N=E^{\prime} N^{\prime}, \quad \text { with } \quad N=\widehat{M} N^{\prime},
$$

where $\widehat{M} \in \mathrm{GL}(2 D+16 ; \mathbb{Z})$. Consequently, $E^{\prime}=E \widehat{M}$ so that

$$
\widehat{M}^{T} \widehat{\eta} \widehat{M}=\widehat{\eta}^{\prime} .
$$

Obviously, only those $\widehat{M} \notin O_{\widehat{\eta}}(D, D+16 ; \mathbb{Z})$ can change the form of the Narain metric. Importantly, all Narain metrics can be reached from $\widehat{\eta}$ given in eq. (2.23) by transformations $\widehat{M} \notin O_{\widehat{\eta}}(D, D+16 ; \mathbb{Z})$. Hence, we assume in the following that the Narain metric $\widehat{\eta}$ is given by eq. (2.23). 


\subsection{Coordinate fields and momenta}

Consider the generalized vielbein in its most general form, i.e. $E=U R \widehat{E} \widehat{M}$, and choose $U=\mathbb{1}$ and $\widehat{M}=\mathbb{1}$, see eq. (2.40). Then, a Narain lattice vector $P$ is represented as

$$
P=\left(\begin{array}{c}
p_{\mathrm{r}} \\
P_{\mathrm{L}}
\end{array}\right)=E N=\left(\begin{array}{c}
\frac{1}{\sqrt{2}} e^{-T}\left(\left(G+C^{T}\right) m-n+A^{T} \alpha_{\mathrm{g}} q\right) \\
\frac{1}{\sqrt{2}} e^{-T}\left(\left(G-C^{T}\right) m+n-A^{T} \alpha_{\mathrm{g}} q\right) \\
\alpha_{\mathrm{g}} q+A m
\end{array}\right) .
$$

It can be thought of to describe both: on the one hand, $L \in \mathbb{\Gamma}$ defines the periodicity for the compactification on a Narain lattice, see eq. (2.7). On the other hand, $P \in \mathbb{\Gamma}$ gives the conjugate momentum, see eq. (2.10).

The matrix $R$ induces the change of right- and left-moving coordinate fields, $y_{\mathrm{r}}, y_{1}$ and $y_{\mathrm{L}}$, to $D$ mixed fields $X, \tilde{X}$ and the remaining 16 left-moving gauge coordinates $X_{\mathrm{g}}$

$$
\widehat{Y}=R^{-1} Y=\left(\begin{array}{ccc}
\frac{1}{\sqrt{2}} \mathbb{1}_{D} & \frac{1}{\sqrt{2}} \mathbb{1}_{D} & 0 \\
\frac{-1}{\sqrt{2}} \mathbb{1}_{D} & \frac{1}{\sqrt{2}} \mathbb{1}_{D} & 0 \\
0 & 0 & \alpha_{\mathrm{g}}^{-1}
\end{array}\right)\left(\begin{array}{c}
y_{\mathrm{r}} \\
y_{\mathrm{l}} \\
y_{\mathrm{L}}
\end{array}\right)=\left(\begin{array}{c}
X \\
\tilde{X} \\
X_{\mathrm{g}}
\end{array}\right)
$$

see eq. (2.3). This relation thus defines which combination of right- and left-moving degrees of freedom are interpreted as the physical coordinates $X$ and which as the dual coordinates $\tilde{X}$. The torus periodicities,

$$
Y \sim Y+E N
$$

read in terms of the coordinates $X$, their duals $\tilde{X}$ and gauge coordinates $X_{\mathrm{g}}$

$$
\left(\begin{array}{c}
X \\
\widetilde{X} \\
X_{\mathrm{g}}
\end{array}\right) \sim\left(\begin{array}{c}
X \\
\widetilde{X} \\
X_{\mathrm{g}}
\end{array}\right)+\widehat{E} N \sim\left(\begin{array}{c}
X \\
\widetilde{X} \\
X_{\mathrm{g}}
\end{array}\right)+\left(\begin{array}{c}
e m \\
e^{-T}\left(n-C^{T} m-A^{T} \alpha_{\mathrm{g}} q\right) \\
q+\alpha_{\mathrm{g}}^{-1} A m
\end{array}\right) .
$$

On-shell the right- and left-moving coordinate fields, $y_{\mathrm{r}}, y_{1}$, have anti-holomorphic and holomorphic mode expansions for a string with boundary condition (2.46) given by

$$
y_{\mathrm{r}}(\bar{\sigma})=y_{\mathrm{r}_{0}}+p_{\mathrm{r}} \bar{\sigma}+\sum_{n \neq 0} \bar{\alpha}_{n} e^{2 \pi i n \bar{\sigma}}, \quad Y_{\mathrm{L}}(\sigma)=Y_{\mathrm{L}_{0}}+P_{\mathrm{L}} \sigma+\sum_{n \neq 0} \alpha_{n} e^{2 \pi i n \sigma},
$$

respectively. Using the change of coordinate field basis (2.45), we see that the conventional coordinate field $X$ and its dual $\widetilde{X}$ have the expansions

$$
\begin{aligned}
& X(\sigma, \bar{\sigma})=\frac{1}{\sqrt{2}}\left(y_{1_{0}}+y_{\mathrm{r}_{0}}\right)+\frac{1}{\sqrt{2}}\left(p_{\mathrm{l}}+p_{\mathrm{r}}\right) \sigma_{1}+\frac{1}{\sqrt{2}}\left(p_{\mathrm{l}}-p_{\mathrm{r}}\right) \sigma_{0}+\text { oscillators } \\
& \widetilde{X}(\sigma, \bar{\sigma})=\frac{1}{\sqrt{2}}\left(y_{\mathrm{1}_{0}}-y_{\mathrm{r}_{0}}\right)+\frac{1}{\sqrt{2}}\left(p_{\mathrm{l}}-p_{\mathrm{r}}\right) \sigma_{1}+\frac{1}{\sqrt{2}}\left(p_{\mathrm{l}}+p_{\mathrm{r}}\right) \sigma_{0}+\text { oscillators }
\end{aligned}
$$

The term linear in the worldsheet space variable $\sigma_{1}$ of $X$ gives the $D$-dimensional winding modes, i.e.

$$
\frac{1}{\sqrt{2}}\left(p_{1}+p_{\mathrm{r}}\right)=e m
$$


The term linear in the worldsheet time variable $\sigma_{0}$ of $X$ corresponds to the $D$-dimensional momentum which is given by

$$
\frac{1}{\sqrt{2}}\left(p_{\mathrm{l}}-p_{\mathrm{r}}\right)=e^{-T}\left(n-C^{T} m-A^{T} \alpha_{\mathrm{g}} q\right)
$$

As expected, for the dual coordinate $\tilde{X}$ the roles of momentum and winding are interchanged.

\section{$3 \quad$ The $T$-duality group}

This section is devoted to exhibit a number of properties of the $T$-duality group. In particular, we develop a convenient basis for this group and parametrize its maximal compact subgroup. In addition, we show that the non-linear transformations of the Narain moduli is a consequence of the coset structure in which the generalized vielbein $\widehat{E}$ lives.

\subsection{Decomposition of the generalized vielbein}

A general $T$-duality transformation is described by an element $\widehat{M} \in \mathrm{O}_{\widehat{\eta}}(D, D+16 ; \mathbb{Z})$. In addition, in eq. (2.39) we parametrized the Narain moduli by the generalized vielbein $\widehat{E} \in \mathrm{O}_{\widehat{\eta}}(D, D+16 ; \mathbb{R})$. Therefore, it is very convenient to describe the properties of matrices $\widehat{M} \in \mathrm{O}_{\widehat{\eta}}(D, D+16 ; \mathbb{R})$ first in general, based on the field of real numbers $\mathbb{R}$. To do so we define a number of specific matrix elements of this group in table 1 . These matrices are chosen such that if we restrict the parameters to be from $\mathbb{Z}$ rather than $\mathbb{R}$, these matrices have only integral entries.

As a first application of the matrices of table 1, we decompose the generalized vielbein (2.39) as a product

$$
\widehat{E}=\widehat{E}(e, B, A)=\widehat{M}_{e}(e) \widehat{M}_{B}(B) \widehat{M}_{A}(A),
$$

of basis matrices $\widehat{M}_{i} \in \mathrm{O}_{\widehat{\eta}}(D, D+16 ; \mathbb{R})$ as given in table 1 . Here, the index $i=e, B, A$ labels the matrix $\widehat{M}_{i}$ and each matrix $\widehat{M}_{i}$ depends on the corresponding kind of Narain moduli $e, B$ and $A$. This parametrization will turn out to be very useful throughout this paper.

\subsection{Coset decomposition of the $T$-duality group}

In section 2.4 we recalled that the moduli space of Narain compactifications can be described geometrically as a coset space (2.35). This already shows the central role that the coset space plays in our discussion and therefore we expand on this property in some detail here.

The generalized vielbein $\widehat{E}$ is an element of the coset

$$
\mathrm{O}(D ; \mathbb{R}) \times \mathrm{O}(D+16 ; \mathbb{R}) \backslash \mathrm{O}_{\widehat{\eta}}(D, D+16 ; \mathbb{R}) .
$$

This means that any element $\widehat{H} \in \mathrm{O}_{\widehat{\eta}}(D, D+16 ; \mathbb{R})$ can be decomposed as

$$
\widehat{H}=\widehat{U} \widehat{E}
$$




\begin{tabular}{|c|c|c|}
\hline & \multicolumn{2}{|c|}{ Parametrizations of subgroups of $\mathrm{O}_{\widehat{\eta}}(D, D+16 ; \mathbb{R})$} \\
\hline \multirow{8}{*}{ 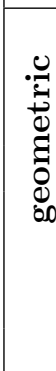 } & $\left(\begin{array}{lll}\Delta K & 0 & 0\end{array}\right)$ & $\left(\begin{array}{lll}\mathbb{1}_{D} & 0 & 0\end{array}\right.$ \\
\hline & 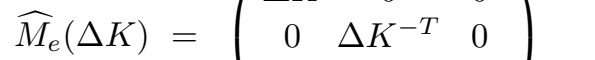 & $\widehat{M}_{W}(\Delta W)=\left(\begin{array}{ccc}0 & \mathbb{1}_{D} & 0\end{array}\right.$ \\
\hline & $\left(\begin{array}{lll}0 & 0 & \mathbb{1}_{16}\end{array}\right)$ & $\left(\begin{array}{ccc}0 & 0 & \alpha_{\mathrm{g}}^{-1} \Delta W \alpha_{\mathrm{g}}\end{array}\right)$ \\
\hline & where $\Delta K \in \mathrm{GL}(D ; \mathbb{R})$ & where $\Delta W \in \mathrm{O}(16 ; \mathbb{R})$ \\
\hline & - $\quad\left(\begin{array}{lll}\mathbb{1}_{D} & 0 & 0\end{array}\right.$ & \\
\hline & $\widehat{M}_{B}(\Delta B)=\left(\begin{array}{ll}\Delta B \mathbb{1}_{D} & 0\end{array}\right)$ & $\widehat{M}_{A}(\Delta A)=\left(-\frac{1}{2} \Delta A^{T} \Delta A \mathbb{1}_{D}-\Delta A^{T} \alpha_{\mathrm{g}}\right.$ \\
\hline & $\left(\begin{array}{lll}0 & 0 & \mathbb{1}_{16}\end{array}\right)$ & $\left(\begin{array}{lll}\alpha_{\mathrm{g}}^{-1} \Delta A & 0 & \mathbb{1}_{16}\end{array}\right)$ \\
\hline & where $\Delta B^{T}=-\Delta B \in \mathrm{M}_{D \times D}(\mathbb{R})$ & $\Delta A \in M_{16 \times D}(\mathbb{R})$ \\
\hline \multirow{7}{*}{ 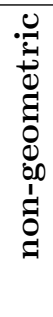 } & $\left(\mathbb{1}_{D}-\epsilon_{i} \epsilon_{i}^{T} \quad \mp \epsilon_{i} \epsilon_{i}^{T}\right.$ & $0 \mathbb{1}_{D}$ \\
\hline & 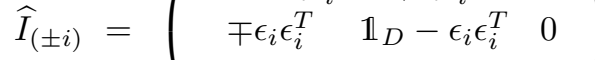 & $\widehat{I}=R^{-1} \eta R=$ \\
\hline & $\left(\begin{array}{lll}0 & 0 & \mathbb{1}_{16}\end{array}\right)$ & $\left(\begin{array}{lll}0 & 0 & \mathbb{1}_{16}\end{array}\right)$ \\
\hline & $\left(\begin{array}{ll}\mathbb{1}_{D} \Delta \beta & 0\end{array}\right)$ & $\left(\mathbb{1}_{D}-\frac{1}{2} \Delta \alpha^{T} \Delta \alpha-\Delta \alpha^{T} \alpha_{\mathrm{g}}\right)$ \\
\hline & $\widehat{M}_{\beta}(\Delta \beta)=$ & $\widehat{M}_{\alpha}(\Delta \alpha)=\quad 0$ \\
\hline & $(0$ & $\left(\begin{array}{ll}0 & \alpha_{\mathrm{g}}^{-1} \Delta \alpha\end{array}\right.$ \\
\hline & where $\Delta \beta^{T}=-\Delta \beta \in \mathrm{M}_{D \times D}(\mathbb{R})$ & $\Delta \alpha \in M_{16 \times D}(\mathbb{R})$ \\
\hline
\end{tabular}

Table 1. This table lists various subgroup elements of the duality group $\mathrm{O}_{\widehat{\eta}}(D, D+16 ; \mathbb{R})$. They are normalized such that if the parameters are taken out of $\mathbb{Z}$ they represent subgroups of $\mathrm{O}_{\widehat{\eta}}\left(D, D+16 ; \mathbb{Z}\right.$ ) (with the additional requirement that $\frac{1}{2} \Delta A^{T} \Delta A$ and $\frac{1}{2} \Delta \alpha^{T} \Delta \alpha$ are integer matrices). The elements listed in the first two rows generate the geometric subgroup $G_{\text {geom }}$ of the duality group. The elements on the third row correspond to true $T$-duality elements that invert one or all radii. Note the difference between $\alpha_{\mathrm{g}}$ and $\Delta \alpha: \alpha_{\mathrm{g}}$ contains the simple roots of $\mathrm{E}_{8} \times \mathrm{E}_{8}$ and is used in the definitions of $\widehat{M}_{W}(\Delta W), \widehat{M}_{A}(\Delta A)$ and $\widehat{M}_{\alpha}(\Delta \alpha)$, while $\Delta \alpha$ is the parameter of $\widehat{M}_{\alpha}(\Delta \alpha)$.

\begin{tabular}{|l|l|}
\hline \multicolumn{3}{|c|}{ Multiplication table of duality subgroup elements } \\
\hline$\widehat{M}_{e}\left(\Delta K^{\prime}\right) \widehat{M}_{e}(\Delta K)=\widehat{M}_{e}\left(\Delta K^{\prime} \Delta K\right)$ & $\widehat{M}_{e}\left(\Delta K^{-T}\right)=\widehat{I} \widehat{M}_{e}(\Delta K) \widehat{I}$ \\
$\widehat{M}_{W}\left(\Delta W^{\prime}\right) \widehat{M}_{W}(\Delta W)=\widehat{M}_{W}\left(\Delta W^{\prime} \Delta W\right)$ & $\widehat{M}_{W}(\Delta W)=\widehat{I} \widehat{M}_{W}(\Delta W) \widehat{I}$ \\
$\widehat{M}_{B}\left(\Delta B^{\prime}\right) \widehat{M}_{B}(\Delta B)=\widehat{M}_{B}\left(\Delta B^{\prime}+\Delta B\right)$ & $\widehat{M}_{\beta}(\Delta \beta)=\widehat{I} \widehat{M}_{B}(\Delta \beta) \widehat{I}=\widehat{M}_{B}(-\Delta \beta)^{T}$ \\
$\widehat{M}_{A}\left(\Delta A^{\prime}\right) \widehat{M}_{A}(\Delta A)=\widehat{M}_{B}(\Delta B) \widehat{M}_{A}\left(\Delta A^{\prime}+\Delta A\right)$ & $\widehat{M}_{\alpha}(\Delta \alpha)=\widehat{I} \widehat{M}_{A}(\Delta \alpha) \widehat{I}=\left(R^{T} R\right)^{-1} \widehat{M}_{A}(-\Delta \alpha)^{T} R^{T} R$ \\
with $\Delta B_{A}=\frac{1}{2}\left(\Delta A^{T} \Delta A^{\prime}-\Delta A^{\prime T} \Delta A\right)$ & \\
\hline$\widehat{M}_{W}(\Delta W) \widehat{M}_{e}(\Delta K)=\widehat{M}_{e}(\Delta K) \widehat{M}_{W}(\Delta W)$ & $\widehat{M}_{W}(\Delta W) \widehat{M}_{B}(\Delta B)=\widehat{M}_{B}(\Delta B) \widehat{M}_{W}(\Delta W)$ \\
$\widehat{M}_{B}(\Delta B) \widehat{M}_{e}(\Delta K)=\widehat{M}_{e}(\Delta K) \widehat{M}_{B}\left(\Delta K^{T} \Delta B \Delta K\right)$ & $\widehat{M}_{W}(\Delta W) \widehat{M}_{A}(\Delta A)=\widehat{M}_{A}(\Delta W \Delta A) \widehat{M}_{W}(\Delta W)$ \\
$\widehat{M}_{A}(\Delta A) \widehat{M}_{e}(\Delta K)=\widehat{M}_{e}(\Delta K) \widehat{M}_{A}(\Delta A \Delta K)$ & $\widehat{M}_{A}(\Delta A) \widehat{M}_{B}(\Delta B)=\widehat{M}_{B}(\Delta B) \widehat{M}_{A}(\Delta A)$ \\
\hline
\end{tabular}

Table 2. Multiplication table for the generators of the duality group $\mathrm{O}_{\widehat{\eta}}(D, D+16 ; \mathbb{R})$.

where the specific standard form (2.39) of the generalized vielbein $\widehat{E}$ lies inside the coset (3.2) and $\widehat{U}=R^{-1} U R$ with $U \in \mathrm{O}(D ; \mathbb{R}) \times \mathrm{O}(D+16 ; \mathbb{R})$ is given in eqs. (2.37) and (2.38).

As this applies to any element of the $T$-duality group, it applies in particular to $\widehat{E} \widehat{M}$ with $\widehat{M} \in \mathrm{O}_{\widehat{\eta}}(D, D+16 ; \mathbb{R})$, i.e.

$$
\widehat{U}_{\widehat{M}} \widehat{E}\left(e^{\prime}, B^{\prime}, A^{\prime}\right)=\widehat{E}(e, B, A) \widehat{M} .
$$

The subscript $\widehat{M}$ of $\widehat{U}_{\widehat{M}}$ emphasizes that the $\mathrm{O}(D ; \mathbb{R}) \times \mathrm{O}(D+16 ; \mathbb{R})$ group element on the left hand side depends on the $T$-duality group element $\widehat{M}$ under consideration. Both 
$\widehat{E}(e, B, A)$ and $\widehat{E}\left(e^{\prime}, B^{\prime}, A^{\prime}\right)$ are given here in the standard form (2.39). This equation (3.4) will be used frequently throughout this paper, for example, when we discuss $T$-duality transformations of Narain moduli in section 3.3 and when we analyze the stabilization of Narain moduli in generalized orbifolds in section 5 .

Simplified standard form of the generalized vielbein. Eq. (3.4) can be used to further simplify the generalized vielbein (2.40): for any discrete $T$-duality element $\widehat{M} \in \mathrm{O}_{\widehat{\eta}}(D, D+16 ; \mathbb{Z}) \subset \mathrm{O}_{\widehat{\eta}}(D, D+16 ; \mathbb{R})$ there is a matrix $U_{\widehat{M}} \in \mathrm{O}(D ; \mathbb{R}) \times \mathrm{O}(D+16 ; \mathbb{R})$ such that eq. (3.4) holds. Consequently, we find

$$
E=U R \widehat{E}(e, B, A) \widehat{M}=\left(U U_{\widehat{M}}\right) R \widehat{E}\left(e^{\prime}, B^{\prime}, A^{\prime}\right)=U^{\prime} R \widehat{E}\left(e^{\prime}, B^{\prime}, A^{\prime}\right),
$$

where $U^{\prime}=U U_{\widehat{M}} \in \mathrm{O}(D ; \mathbb{R}) \times \mathrm{O}(D+16 ; \mathbb{R})$ is arbitrary since $U$ is arbitrary. Relabelling our expression by removing the primes we obtain the most general from of the generalized vielbein as

$$
E=U R \widehat{E}(e, B, A),
$$

where $\widehat{E}(e, B, A)$ is given in eqs. (2.39) and (3.1) and $U \in \mathrm{O}(D ; \mathbb{R}) \times \mathrm{O}(D+16 ; \mathbb{R})$ may be chosen freely.

Compact subgroup in the coset decomposition. In what follows, we consider eq. (3.4) and first compute the explicit matrix expression of $U_{\widehat{M}} \in \mathrm{O}(D ; \mathbb{R}) \times \mathrm{O}(D+16 ; \mathbb{R})$, and determine the transformed moduli, $e^{\prime}, B^{\prime}, A^{\prime}$, in terms of $\widehat{M}$ and the initial moduli $e$, $B$ and $A$.

To do so, we decompose $\widehat{M}$ into its $3 \times 3$-block structure, i.e.

$$
\widehat{M}=\left(\begin{array}{lll}
\widehat{M}_{11} & \widehat{M}_{12} & \widehat{M}_{13} \\
\widehat{M}_{21} & \widehat{M}_{22} & \widehat{M}_{23} \\
\widehat{M}_{31} & \widehat{M}_{32} & \widehat{M}_{33}
\end{array}\right),
$$

where $\widehat{M}_{11}, \widehat{M}_{12}, \widehat{M}_{21}$ and $\widehat{M}_{22}$ are $D \times D$-matrices, $\widehat{M}_{13}$ as well as $\widehat{M}_{23}$ are $D \times 16$-matrices, $\widehat{M}_{31}$ as well as $\widehat{M}_{32}$ are $16 \times D$-matrices, while $\widehat{M}_{33}$ is a $16 \times 16$-matrix, respectively. Furthermore, in order to avoid lengthy formulae, we introduce short-hand notations

$$
\begin{aligned}
& \widehat{M}_{1}=-\widehat{M}_{21}+\left(G+C^{T}\right) \widehat{M}_{11}+A^{T} \alpha_{\mathrm{g}} \widehat{M}_{31}, \\
& \widehat{M}_{2}=-\widehat{M}_{22}+\left(G+C^{T}\right) \widehat{M}_{12}+A^{T} \alpha_{\mathrm{g}} \widehat{M}_{32}, \\
& \widehat{M}_{3}=-\widehat{M}_{23}+\left(G+C^{T}\right) \widehat{M}_{13}+A^{T} \alpha_{\mathrm{g}} \widehat{M}_{33},
\end{aligned}
$$

which will recur frequently throughout the rest of this work. Next, we compute the products of matrices contained in eq. (3.4), i.e.

$$
\widehat{E}(e, B, A) \widehat{M} \quad \text { and } \quad \widehat{U}_{\widehat{M}} \widehat{E}\left(e^{\prime}, B^{\prime}, A^{\prime}\right)
$$

where each matrix is given in its $3 \times 3$-block structure, e.g. $\widehat{U}_{\widehat{M}}$ is given in eq. (2.38). The result is set equal which yields $3 \times 3=9$ equations from eq. (3.4). By doing so, we can 
solve for the blocks of $U_{\widehat{M}}=R \widehat{U}_{\widehat{M}} R^{-1}$ as defined in eq. (2.38) and obtain

$$
\begin{aligned}
u_{\mathrm{l}} & =\left(\mathbb{1}_{D}-2 e \widehat{M}_{12} \widehat{M}_{2}^{-1} e^{T}\right) u_{\mathrm{r}}, \\
u_{\mathrm{L}} & =\sqrt{2} e\left(\widehat{M}_{13}-\widehat{M}_{12} \widehat{M}_{2}^{-1} \widehat{M}_{3}\right) \alpha_{\mathrm{g}}^{-1}, \\
u_{\mathrm{Ll}} & =-\sqrt{2}\left(\alpha_{\mathrm{g}} \widehat{M}_{32}+A \widehat{M}_{12}\right) \widehat{M}_{2}^{-1} e^{T} u_{\mathrm{r}}, \\
u_{\mathrm{L}} & =A \widehat{M}_{13} \alpha_{\mathrm{g}}^{-1}+\alpha_{\mathrm{g}} \widehat{M}_{33} \alpha_{\mathrm{g}}^{-1}-\left(A \widehat{M}_{12}+\alpha_{\mathrm{g}} \widehat{M}_{32}\right) \widehat{M}_{2}^{-1} \widehat{M}_{3} \alpha_{\mathrm{g}}^{-1},
\end{aligned}
$$

for arbitrary $u_{\mathrm{r}} \in \mathrm{O}(D ; \mathbb{R})$. We have checked explicitly that these equations give a matrix $U$ such that the conditions (2.36) are satisfied. Let us remark one observations from eq. (3.10a): $\widehat{M}_{12} \neq 0$ is a necessary condition for $u_{\mathrm{r}} \neq u_{\mathrm{l}}$. In other words, if $\widehat{M}_{12}=0$ then $u_{\mathrm{r}}=u_{\mathrm{l}}$. In addition, let us stress that these equations (3.10) will become very important later in the context of Narain orbifolds where $U$ becomes the orbifold twist $\Theta$, for example in section 5.2. Furthermore, we identify the following three expressions

$$
\begin{aligned}
\widehat{M}_{2}^{T} & =-\left(e^{\prime-1} u_{\mathrm{r}}^{-1} e\right), \\
A^{\prime}\left(e^{\prime-1} u_{\mathrm{r}}^{-1} e\right) & =\alpha_{\mathrm{g}}^{-T} \widehat{M}_{3}^{T},
\end{aligned}
$$

from eq. (3.4), which we use in the following discussion.

\subsection{Transformation of Narain moduli}

Using the coset decomposition discussed above, we can derive the transformation properties of the Narain moduli $G, B$ and $A$ under general $T$-duality transformations $\widehat{M} \in \mathrm{O}_{\widehat{\eta}}(D, D+16 ; \mathbb{R})$. Using the results of section 2.4 we see that the generalized vielbein (3.1) transforms under $\widehat{M}$ as

$$
\widehat{E}(e, B, A) \mapsto \widehat{E}\left(e^{\prime}, B^{\prime}, A^{\prime}\right)=\widehat{U}_{\widehat{M}}^{-1} \widehat{E}(e, B, A) \widehat{M},
$$

where $\widehat{U}_{\widehat{M}}=R^{-1} U_{\widehat{M}} R$ and $U_{\widehat{M}} \in \mathrm{O}(D ; \mathbb{R}) \times \mathrm{O}(D+16 ; \mathbb{R})$. In other words, assume we have given a $T$-duality transformation $\widehat{M} \in \mathrm{O}_{\widehat{\eta}}(D, D+16 ; \mathbb{R})$. Then, there exists a matrix $U_{\widehat{M}}$ as given in eq. (3.10) such that $\widehat{E}\left(e^{\prime}, B^{\prime}, A^{\prime}\right)$ is in the standard form (3.1).

Hence, we are able to identify the transformation properties of $e, G+C^{T}$ and $A$ under general $T$-duality transformations from eq. (3.11). We find

$$
e^{\prime}=-u_{\mathrm{r}}^{-1} e \widehat{M}_{2}^{-T} \quad, \quad G^{\prime}+C^{\prime T}=-\widehat{M}_{2}^{-1} \widehat{M}_{1} \quad \text { and } \quad A^{\prime}=-\alpha_{\mathrm{g}}^{-T} \widehat{M}_{3}^{T} \widehat{M}_{2}^{-T} \text {, }
$$

where $u_{\mathrm{r}}^{T} u_{\mathrm{r}}=\mathbb{1}_{D}$. These transformations can be expanded out (by taking the antisymmetric part of $G^{\prime}+C^{\prime T}$ to solve for $B^{\prime}$ ) and we obtain the transformations of the moduli $G, B, A$, i.e.

$$
\begin{array}{rlrl}
G \mapsto G^{\prime} & =\widehat{M}_{2}^{-1} G \widehat{M}_{2}^{-T}, & B \mapsto B^{\prime}=\frac{1}{2}\left(\widehat{M}_{2}^{-1} \widehat{M}_{1}-\widehat{M}_{1}^{T} \widehat{M}_{2}^{-T}\right), \\
A \mapsto A^{\prime} & =-\alpha_{\mathrm{g}}^{-T} \widehat{M}_{3}^{T} \widehat{M}_{2}^{-T}, & &
\end{array}
$$

using the short-hands defined in eq. (3.8). This generalizes the results for $\mathrm{O}(D, D)$ (see e.g. [85]) to the heterotic case [86]. As a cross-check, using $\widehat{M} \widehat{\eta}^{-1} \widehat{M}^{T}=\widehat{\eta}^{-1}$ one can show that eq. (3.14) yields $G^{\prime}+C^{\prime T}=-\widehat{M}_{2}^{-1} \widehat{M}_{1}$ as given in eq. (3.13). 


\subsection{Specific elements of the $T$-duality group}

Next, we discuss various elements and subgroups of the group $\mathrm{O}_{\widehat{\eta}}(D, D+16 ; \mathbb{R})$ in detail and analyze their actions on the Narain moduli $G, B, A$. The parametrizations of these subgroups can be found in table 1 and their most important products are given in table 2 .

\subsubsection{The geometric subgroup}

The elements $\widehat{M}_{e}, \widehat{M}_{W}, \widehat{M}_{A}$ and $\widehat{M}_{B}$ as listed in table 1 generate a subgroup of $\mathrm{O}_{\widehat{\eta}}(D, D+16 ; \mathbb{R})$ which we denote by $G_{\text {geom }}(\mathbb{R})$. This is the largest $T$-duality subgroup, that still admits a standard geometrical interpretation, hence the name: geometric subgroup. In more detail, all elements $\widehat{M}_{\text {geom }} \in G_{\text {geom }}(\mathbb{R})$ can be cast to the form

$$
\widehat{M}_{\text {geom }}=\widehat{M}_{W}(\Delta W) \widehat{M}_{e}(\Delta K) \widehat{M}_{B}(\Delta B) \widehat{M}_{A}(\Delta A)
$$

Then, using the results of section 2.4 we see that the generalized vielbein (3.1) transforms under $\widehat{M}_{\text {geom }}$ as

$$
\widehat{E}(e, B, A) \mapsto \widehat{E}\left(e^{\prime}, B^{\prime}, A^{\prime}\right)=\widehat{U}_{\text {geom }}^{-1} \widehat{E}(e, B, A) \widehat{M}_{\text {geom }},
$$

where

$$
\begin{aligned}
e^{\prime} & =\left(u_{\mathrm{r}}^{\text {geom }}\right)^{-1} e \Delta K, \\
B^{\prime} & =\Delta K^{T} B \Delta K+\Delta B+\frac{1}{2}\left(\Delta A^{T} \Delta W^{T} A \Delta K-\Delta K^{T} A^{T} \Delta W \Delta A\right), \\
A^{\prime} & =\Delta W^{T} A \Delta K+\Delta A .
\end{aligned}
$$

Here $\widehat{U}_{\text {geom }}=R^{-1} U_{\text {geom }} R$ and $U_{\text {geom }} \in \mathrm{O}(D ; \mathbb{R}) \times \mathrm{O}(D+16 ; \mathbb{R})$ must be chosen such that $\widehat{E}\left(e^{\prime}, B^{\prime}, A^{\prime}\right)$ is given in the standard form (3.1). Furthermore, in eq. (3.16) we have used various group multiplication properties as given in table 2 to compute the product $\widehat{E}(e, B, A) \widehat{M}_{\text {geom }}$ (analogously, one could have used the general transformations (3.13) and (3.14) for $\widehat{M}=\widehat{M}_{\text {geom }}$ to derive eq. (3.16)). Notice that under a $\widehat{M}_{W}(\Delta W)$-transformation the form of the generalized vielbein is not strictly preserved. Nevertheless, it is of the correct form such that it can be absorbed by the choice of $\widehat{U}_{\text {geom }}=\widehat{M}_{W}(\Delta W) \widehat{M}_{e}\left(u_{\mathrm{r}}^{\text {geom }}\right)$, i.e.

$$
U_{\text {geom }}=R \widehat{M}_{W}(\Delta W) \widehat{M}_{e}\left(u_{\mathrm{r}}^{\text {geom }}\right) R^{-1}=\left(\begin{array}{lll}
u_{\mathrm{r}}^{\text {geom }} & & \\
& u_{\mathrm{r}}^{\text {geom }} & \\
& & \Delta W
\end{array}\right),
$$

since $U_{\text {geom }}$ is an element of $\mathrm{O}(D ; \mathbb{R}) \times \mathrm{O}(D+16 ; \mathbb{R})$ because $\Delta W \in \mathrm{O}(16 ; \mathbb{R})$ and $u_{\mathrm{r}}^{\text {geom }} \in \mathrm{O}(D ; \mathbb{R})$.

In the following, we give details for various elements of the $T$-duality group. We start with the four generators $\widehat{M}_{e}, \widehat{M}_{W}, \widehat{M}_{A}$ and $\widehat{M}_{B}$ of the geometric subgroup $G_{\text {geom }}(\mathbb{R})$ and use eqs. (3.16) in order to compute the transformation of moduli. 
Change of geometrical basis $\widehat{M}_{e}(\Delta K)$. Changes of the geometrical basis $e$ are given by $\widehat{M}_{e}(\Delta K)$ with $\Delta K \in \mathrm{GL}(D ; \mathbb{R})$. The unit element $\widehat{M}_{e}(\Delta K)=\mathbb{1}$ has $\Delta K=\mathbb{1}_{D}$. From eqs. (3.16) we identify the transform of the background fields $G, B$ and $A$ : $\widehat{M}_{e}(\Delta K)$ leads to a change of basis of the $D$-dimensional torus, $e \mapsto e^{\prime}=\left(u_{\mathrm{r}}^{\text {geom }}\right)^{-1} e \Delta K$, and

$$
G \mapsto G^{\prime}=\Delta K^{T} G \Delta K, \quad B \mapsto B^{\prime}=\Delta K^{T} B \Delta K, \quad A \mapsto A^{\prime}=A \Delta K .
$$

Change of basis in the gauge degrees of freedom $\widehat{M}_{W}(\Delta W)$. In addition, we may change the basis in the gauge degrees of freedom by $\widehat{M}_{W}(\Delta W)$ with $\Delta W \in \mathrm{O}(16 ; \mathbb{R})$. The unit element $\widehat{M}_{W}(\Delta W)=\mathbb{1}$ has $\Delta W=\mathbb{1}_{16} \cdot \widehat{M}_{W}(\Delta W)$ induces a transformation

$$
A \mapsto A^{\prime}=\Delta W^{T} A
$$

of the Wilson lines, while $G$ and $B$ remain invariant.

In the case of the discrete $T$-duality group we define $\rho_{W}=\alpha_{\mathrm{g}}^{-1} \Delta W \alpha_{\mathrm{g}}$. Then, $\widehat{M}_{W}(\Delta W) \in \mathrm{O}_{\widehat{\eta}}(D, D+16 ; \mathbb{Z})$ if $\rho_{W} \in \mathrm{O}_{g}(16 ; \mathbb{Z})$, i.e. $\rho_{W}^{T} g \rho_{W}=g$ using $g=\alpha_{\mathrm{g}}^{T} \alpha_{\mathrm{g}}$. Hence, $\rho_{W}$ is an automorphism of the $\mathrm{E}_{8} \times \mathrm{E}_{8}$ root lattice spanned by $\alpha_{\mathrm{g}}$.

$\boldsymbol{B}$-field shifts $\widehat{\boldsymbol{M}}_{\boldsymbol{B}}(\boldsymbol{\Delta} \boldsymbol{B})$. Matrices of the form $\widehat{M}_{B}(\Delta B)$ with $\Delta B^{T}=-\Delta B \in \mathrm{M}_{D \times D}(\mathbb{R})$ leave $G$ and $A$ invariant and only induce $B$-field shifts, i.e.

$$
B \mapsto B^{\prime}=B+\Delta B .
$$

$B$-field shifts generate a subgroup $G_{B}(\mathbb{R}) \subset \mathrm{O}_{\widehat{\eta}}(D, D+16 ; \mathbb{R})$. The unit element $\widehat{M}_{B}(\Delta B)=\mathbb{1}$ is given by $\Delta B=0$.

Wilson line shifts $\widehat{M}_{A}(\Delta A)$. Wilson line shifts are generated by $\widehat{M}_{A}(\Delta A)$ with $\alpha_{\mathrm{g}}^{-1} \Delta A \in M_{16 \times D}(\mathbb{R})$. Indeed, we obtain

$$
A \mapsto A^{\prime}=A+\Delta A, \quad B \mapsto B^{\prime}=B+\frac{1}{2}\left(\Delta A^{T} A-A^{T} \Delta A\right) .
$$

Hence, transformations of the Wilson lines $A$ are accompanied by a $B$-field transformation, while the metric $G$ is kept invariant. Furthermore, we find

$$
\widehat{M}_{A}\left(\Delta A^{\prime}\right) \widehat{M}_{A}(\Delta A)=\widehat{M}_{B}\left(\Delta B{ }_{A}\right) \widehat{M}_{A}\left(\Delta A^{\prime}+\Delta A\right),
$$

with $\Delta B_{A}=\frac{1}{2}\left(\Delta A^{T} \Delta A^{\prime}-\Delta A^{\prime T} \Delta A\right)$, where we remark that Wilson line shifts and $B$-field shifts commute, see table 2 .

Due to eq. (3.22), Wilson line shifts do not generate a subgroup of $\mathrm{O}_{\widehat{\eta}}(D, D+16 ; \mathbb{R})$ on their own, but only when combined with $B$-field shifts $\widehat{M}_{B}(\Delta B)$. We denote this subgroup by $G_{\mathrm{WL}}(\mathbb{R})$. The subgroup $G_{B}(\mathbb{R})$ of $B$-field shifts and the subgroup $G_{\mathrm{WL}}(\mathbb{R})$ of combined Wilson line and $B$-field shifts are both normal subgroups of the geometric subgroup $G_{\text {geom }}(\mathbb{R})$. In particular, it follows that

$$
G_{\text {geom }} / G_{\mathrm{WL}}=\mathrm{GL}(D ; \mathbb{R}) \times \mathrm{O}(16 ; \mathbb{R}) .
$$

Note that $\widehat{M}_{B}(\Delta B) \widehat{M}_{A}(\Delta A)$ with $\Delta B \notin \mathrm{M}_{D \times D}(\mathbb{Z})$ can be an element of the discrete $T$-duality group, i.e. $\widehat{M}_{B}(\Delta B) \widehat{M}_{A}(\Delta A) \in \mathrm{O}_{\widehat{\eta}}(D, D+16 ; \mathbb{Z})$, if $\alpha_{\mathrm{g}}^{-1} \Delta A \in M_{16 \times D}(\mathbb{Z})$ and

$$
-\frac{1}{2} \Delta A^{T} \Delta A+\Delta B \in M_{D \times D}(\mathbb{Z}) .
$$




\subsubsection{Non-geometric elements}

In the following, we give details for non-geometric elements of the $T$-duality group. We use eqs. (3.14) in order to compute the transformation of moduli.

$\boldsymbol{T}$-duality inversions. We can define $\mathbb{Z}_{2}$ involutions

$$
\widehat{I}_{( \pm i)}=\left(\begin{array}{ccc}
\mathbb{1}_{D}-\epsilon_{i} \epsilon_{i}^{T} & \mp \epsilon_{i} \epsilon_{i}^{T} & 0 \\
\mp \epsilon_{i} \epsilon_{i}^{T} & \mathbb{1}_{D}-\epsilon_{i} \epsilon_{i}^{T} & 0 \\
0 & 0 & \mathbb{1}_{16}
\end{array}\right) \text { for } i=1, \ldots, D
$$

where $\epsilon_{i}$ denotes the standard basis vector in the $i$-th torus direction. The element $\widehat{I}_{( \pm i)}$ can be written as conjugation of a reflection in the $i$-th left- or right-moving direction as $\widehat{I}_{( \pm i)}=R^{-1} I_{( \pm i)} R$ using

$$
I_{(+i)}=\left(\begin{array}{ccc}
\mathbb{1}_{D} & 0 & 0 \\
0 & \mathbb{1}_{D}-2 \epsilon_{i} \epsilon_{i}^{T} & 0 \\
0 & 0 & \mathbb{1}_{16}
\end{array}\right), \quad I_{(-i)}=\left(\begin{array}{ccc}
\mathbb{1}_{D}-2 \epsilon_{i} \epsilon_{i}^{T} & 0 & 0 \\
0 & \mathbb{1}_{D} & 0 \\
0 & 0 & \mathbb{1}_{16}
\end{array}\right)
$$

Therefore, all the elements $\widehat{I}_{( \pm i)}$ can be obtained from $\widehat{I}_{( \pm 1)}$ by conjugation with an appropriate change of basis element $\widehat{M}_{e}(\Delta K)$.

The element $\widehat{I}_{(-i)}$ induces a $T$-duality inversion along the $i$-th torus direction. We can preform the $T$-duality inversion in all torus directions simultaneously by

$$
\widehat{I}=\widehat{I}_{(-1)} \cdots \widehat{I}_{(-D)}
$$

as given in table 1. Using the general results (3.14) we find for this element

$$
\begin{aligned}
G & \mapsto G^{\prime}=\widehat{M}_{2}^{-1} G \widehat{M}_{2}^{-T}, \\
B & \mapsto B^{\prime}=-\widehat{M}_{2}^{-1} B \widehat{M}_{2}^{-T}, \\
A & \mapsto A^{\prime}=-A \widehat{M}_{2}^{-T},
\end{aligned}
$$

where $\widehat{M}_{2}=G+C^{T}$. For $A=0$ we get $\widehat{M}_{2}=G-B$. Hence, eq. (3.28) yields the famous transformation $(G+B) \mapsto(G+B)^{-1}$.

Maximal subgroup of $\mathrm{O}_{\widehat{\eta}}(D, D+16 ; \mathbb{R})$ connected to the identity. As an application of the special duality elements $\widehat{I}_{( \pm i)}$ we discuss the maximal non-compact subgroup $\mathrm{SO}_{\widehat{\eta}}^{+}(D, D+16 ; \mathbb{R})$ of the $T$-duality group $\mathrm{O}_{\widehat{\eta}}(D, D+16 ; \mathbb{R})$ that is connected to the identity. The quotient group

$$
\mathrm{O}_{\widehat{\eta}}(D, D+16 ; \mathbb{R}) / \mathrm{SO}_{\widehat{\eta}}^{+}(D, D+16 ; \mathbb{R}) \cong \mathbb{Z}_{2} \times \mathbb{Z}_{2}
$$

is of order four and, hence, corresponds to four disconnected components of $O_{\widehat{\eta}}(D, D+16 ; \mathbb{R})$ : one can choose the two $\mathbb{Z}_{2}$-generators as the elements $\widehat{I}_{(-1)}$ and $\widehat{I}_{(+1)}$. The matrix representations of the four disconnected components are obtained by multiplying $\mathbb{1}, \widehat{I}_{(-1)}, \widehat{I}_{(+1)}$ or $\widehat{I}_{(-1)} \widehat{I}_{(+1)}$ by arbitrary matrices of $\operatorname{SO}_{\widehat{\eta}}^{+}(D, D+16 ; \mathbb{R})$. 
Inverted $\boldsymbol{B}$-field shifts $\widehat{\boldsymbol{M}}_{\boldsymbol{\beta}}(\boldsymbol{\Delta} \boldsymbol{\beta})$. Even though the following two elements $\widehat{M}_{\beta}(\Delta \beta)$ and $\widehat{M}_{\alpha}(\Delta \alpha)$ can be obtained by combining the $B$ - and $A$-shifts with the inversion element $\widehat{I}$, we list them explicitly as they are important in the context of non-geometry.

Inverted $B$-field shifts, often referred to as $\beta$-transformations, are generated by

$$
\widehat{M}_{\beta}(\Delta \beta)=\widehat{I} \widehat{M}_{B}(\Delta \beta) \widehat{I},
$$

with $\Delta \beta^{T}=-\Delta \beta \in M_{D \times D}(\mathbb{R})$. The $\beta$-transformations of the metric, $B$-field and gauge backgrounds take the form

$$
\begin{aligned}
G \mapsto G^{\prime} & =\widehat{M}_{2}^{-1} G \widehat{M}_{2}^{-T}, \quad A \mapsto A^{\prime}=-A \widehat{M}_{2}^{-T}, \\
B \mapsto B^{\prime} & =\widehat{M}_{2}^{-1}\left(B-\left(G+C^{T}\right) \Delta \beta(G+C)\right) \widehat{M}_{2}^{-T},
\end{aligned}
$$

using $\widehat{M}_{2}=-\mathbb{1}_{D}+\left(G+C^{T}\right) \Delta \beta$ in eq. (3.14).

Inverted Wilson line shifts $\widehat{M}_{\alpha}(\Delta \alpha)$. Finally, by inverting the Wilson line shifts $\widehat{M}_{A}$ we obtain

$$
\widehat{M}_{\alpha}(\Delta \alpha)=\widehat{I} \widehat{M}_{A}(\Delta \alpha) \widehat{I}
$$

with $\alpha_{\mathrm{g}}^{-1} \Delta \alpha \in M_{16 \times D}(\mathbb{R})$.

The inversion of changes of bases, i.e. $\widehat{I} \widehat{M}_{e}(\Delta K) \widehat{I}$ and $\widehat{I} \widehat{M}_{W}(\Delta W) \widehat{I}$, just become changes of bases again. Hence, they do not give us novel transformations. For completeness we nevertheless list them in table 2. Indeed, counting the number of generators shows that this list contains all possible $\mathrm{O}_{\widehat{\eta}}(D, D+16, \mathbb{R})$ transformations.

\subsection{The maximal compact subgroup of $\mathrm{O}_{\widehat{\eta}}(D, D+16 ; \mathbb{R})$}

Next, we discuss the maximal compact subgroup of $\mathrm{O}_{\widehat{\eta}}(D, D+16 ; \mathbb{R})$. To do so, we note that the maximal compact subgroup of $\mathrm{O}_{\eta}(D, D+16 ; \mathbb{R})$ is $\mathrm{O}(D ; \mathbb{R}) \times \mathrm{O}(D+16 ; \mathbb{R})$. By conjugation with $R$ one maps elements $U \in \mathrm{O}_{\eta}(D, D+16 ; \mathbb{R})$ one-to-one to elements $\widehat{U} \in \mathrm{O}_{\widehat{\eta}}(D, D+16 ; \mathbb{R})$, i.e. $\widehat{U}=R^{-1} U R$. Thus, the maximal compact subgroup of $\mathrm{O}_{\widehat{\eta}}(D, D+16 ; \mathbb{R})$ is also $\mathrm{O}(D ; \mathbb{R}) \times \mathrm{O}(D+16 ; \mathbb{R})$. An explicit parametrization of this subgroup is given by $\widehat{U}$ in eq. (2.38). Note that, as discussed in section 2.4, elements $U \in \mathrm{O}(D ; \mathbb{R}) \times \mathrm{O}(D+16 ; \mathbb{R}) \subset \mathrm{O}_{\eta}(D, D+16 ; \mathbb{R})$ map physically identical Narain configurations to each other.

Using the generators of the $\mathrm{O}_{\widehat{\eta}}(D, D+16 ; \mathbb{R})$ listed in table 1 an element $U$ from the identity component of $\mathrm{O}(D ; \mathbb{R}) \times \mathrm{O}(D+16 ; \mathbb{R})$ defined by eq. (2.36) can be expressed as follows $U=R \widehat{U} R^{-1}$;

$$
\widehat{U}=\widehat{M}_{e}(\Delta \theta) \widehat{M}_{W}(\Delta W) \widehat{M}_{\alpha}(\Delta A) \widehat{M}_{\beta}(\Delta B) \widehat{M}_{e}(\Delta K) \widehat{M}_{A}(\Delta A) \widehat{M}_{B}(\Delta B),
$$

if $u_{+}$is invertible, see eq. (2.38), and we defined

$$
\begin{array}{rlrl}
\Delta K & =\mathbb{1}_{D}+\Delta C, & \Delta C & =\Delta B+\frac{1}{2} \Delta A^{T} \Delta A, \quad \Delta B^{T}=-\Delta B, \\
\Delta \theta^{T} \Delta \theta & =\mathbb{1}_{D} \quad \text { and } \quad \Delta W^{T} \Delta W & =\mathbb{1}_{16} .
\end{array}
$$


The first two factors $\widehat{M}_{e}(\Delta \theta) \widehat{M}_{W}(\Delta W)$ in eq. (3.33) define the subgroup $\mathrm{O}(D ; \mathbb{R}) \times$ $\mathrm{O}(16 ; \mathbb{R})$, where the $\mathrm{O}(D ; \mathbb{R})$-factor lies diagonally in both the left- and right-moving directions. This can be seen from eq. (3.33) by using the expressions for the duality group elements given in table 1 and the matrix $R$ defined in eq. (2.26). Then, we obtain

$$
U=\left(\begin{array}{ccc}
\Delta \theta & 0 & 0 \\
0 & \Delta \theta & 0 \\
0 & 0 & \Delta W
\end{array}\right)\left(\begin{array}{ccc}
\mathbb{1}_{D} & 0 & 0 \\
0 & \left(\mathbb{1}_{D}-\Delta C\right)^{T}\left(\mathbb{1}_{D}+\Delta C\right)^{-T} & -\sqrt{2}\left(\mathbb{1}_{D}+\Delta C\right)^{-T} \Delta A^{T} \\
0 & \sqrt{2} \Delta A\left(\mathbb{1}_{D}+\Delta C\right)^{-T} & \mathbb{1}_{16}-\Delta A\left(\mathbb{1}_{D}+\Delta C\right)^{-T} \Delta A^{T}
\end{array}\right)
$$

By comparing this with eq. (2.37) one can read off the expressions for the submatrices

$$
\begin{aligned}
u_{\mathrm{l}} & =\Delta \theta\left(\mathbb{1}_{D}-\Delta C\right)^{T}\left(\mathbb{1}_{D}+\Delta C\right)^{-T}, & u_{\mathrm{lL}} & =-\sqrt{2} \Delta \theta\left(\mathbb{1}_{D}+\Delta C\right)^{-T} \Delta A^{T}, \\
u_{\mathrm{Ll}} & =\sqrt{2} \Delta W \Delta A\left(\mathbb{1}_{D}+\Delta C\right)^{-T}, & u_{\mathrm{L}} & =\Delta W\left(\mathbb{1}_{16}-\Delta A\left(\mathbb{1}_{D}+\Delta C\right)^{-T} \Delta A^{T}\right)
\end{aligned}
$$

and $u_{\mathrm{r}}=\Delta \theta$. One can verify that these expressions satisfy the constraints (2.36).

In addition, for a given element $U \in \mathrm{O}(D ; \mathbb{R}) \times \mathrm{O}(D+16 ; \mathbb{R})$ one can use eq. (3.35) to decompose $\widehat{U}=R^{-1} U R$ according to eq. (3.33), i.e.

$$
\begin{aligned}
\Delta \theta & =u_{\mathrm{r}}, & \Delta C & =-u_{-}^{T} u_{+}^{-T} \\
\Delta A & =-\frac{1}{\sqrt{2}} u_{\mathrm{lL}}^{T} u_{+}^{-T}, & \Delta W & =u_{\mathrm{L}}\left(\mathbb{1}_{16}-\frac{1}{2} u_{\mathrm{lL}}^{T} u_{+}^{-T} u_{\mathrm{r}}^{-1} u_{\mathrm{lL}}\right)^{-1}
\end{aligned}
$$

where we assumed that $u_{+}$is invertible.

\section{Generalized space groups of Narain orbifolds}

In this section we introduce the generalized space group for heterotic Narain orbifolds and discuss some of its properties. In particular, we define orbifold projections to characterize quantization conditions of the generalized shift vectors and state the conditions to preserve $\mathcal{N}=1$ supersymmetry.

\subsection{Heterotic Narain orbifolds}

Next, we consider orbifolds of the heterotic Narain lattice construction denoted by

$$
T_{\mathbb{\Gamma}}^{2 D+16} / \mathbf{P}
$$

Here, the $2 D+16$-dimensional torus $T_{\mathbb{\Gamma}}^{2 D+16}$ is specified by a Narain lattice $\mathbb{\Gamma}$. In addition, the Narain point group $\mathbf{P}$ is defined as a (sub-)group of the rotational symmetries of $\mathbb{\Gamma}$, as we will see later in eq. (4.13). Hence, the Narain point group $\mathbf{P}$ is finite. The generators of $\mathbf{P}$ are $(2 D+16) \times(2 D+16)$ matrices and they are denoted by $\Theta_{\alpha}$, for $\alpha=1, \ldots, N_{\mathbf{P}}$. $K_{\alpha}$ is the order of $\Theta_{\alpha}$. In more detail, for each generator $\Theta_{\alpha}$, the order $K_{\alpha}$ is the smallest non-negative integer such that $\Theta_{\alpha}^{K_{\alpha}}=\mathbb{1}$. Elements of $\mathbf{P}$ are often called twists. In the following, a generic twist will be denoted by $\Theta$ and $K$ gives its order. 
To define the compactification of the heterotic string on a Narain orbifold [40, 53], the main idea is to generalize the boundary conditions (2.7) of the $2 D+16$-dimensional rightand left-moving coordinate-vector $Y$ to

$$
Y(\sigma+1, \bar{\sigma}+1)=\Theta Y(\sigma, \bar{\sigma})+V_{\Theta}+L,
$$

for all elements $\Theta \in \mathbf{P}$ and $L \in \mathbb{\Gamma}$. In general, for each twist $\Theta$ there is a so-called generalized shift $V_{\Theta}$ associated to it, which will be discussed in detail later. Importantly, the twists $\Theta$ are not allowed to mix right- and left-moving fields in eq. (4.2). Hence, for all $\Theta \in \mathbf{P}$ we demand

$$
\Theta=\left(\begin{array}{cc}
\theta_{\mathrm{r}} & 0 \\
0 & \Theta_{\mathrm{L}}
\end{array}\right) \in O(D ; \mathbb{R}) \times O(D+16 ; \mathbb{R}) .
$$

Consequently, we find the conditions

$$
\Theta_{\alpha}^{T} \Theta_{\alpha}=\mathbb{1}, \quad \Theta_{\alpha}^{T} \eta \Theta_{\alpha}=\eta \quad \text { and } \quad \Theta_{\alpha}^{K_{\alpha}}=0,
$$

for all generators $\Theta_{\alpha}$ of the Narain point group.

Furthermore, we call a Narain orbifold symmetric $[5,6]$, if there is a basis such that all generators $\Theta_{\alpha} \in \mathbf{P}$ are simultaneously of the form

$$
\Theta_{\alpha}=\left(\begin{array}{ccc}
\theta_{\alpha} & 0 & 0 \\
0 & \theta_{\alpha} & 0 \\
0 & 0 & \mathbb{1}_{16}
\end{array}\right) \in O(D ; \mathbb{R}) \subset O(D ; \mathbb{R}) \times O(D+16 ; \mathbb{R})
$$

If such a basis does not exist, then the corresponding Narain orbifold is asymmetric. Even though this definition of symmetric orbifolds involves a choice of basis, this property is in fact basis independent. Nevertheless, in a given basis it might be difficult to see whether a Narain orbifold is symmetric or asymmetric: one can bring a symmetric twist $\Theta_{\text {sym }}$ into a seemingly asymmetric twist $\Theta_{\text {asym }}=U^{-1} \Theta_{\text {sym }} U$ by the choice of $U \in \mathrm{O}(D ; \mathbb{R}) \times \mathrm{O}(D+16 ; \mathbb{R})$, see also the example in section 8.3. However, the conjugation with $U$ can neither change the orders of $\theta_{\mathrm{r}}$ and $\Theta_{\mathrm{L}}$, nor the two finite groups which are generated by either $\theta_{\alpha \mathrm{r}}$ or $\Theta_{\alpha \mathrm{L}}$.

\subsection{Generalized space group}

It has been proven to be very convenient to employ a space group formulation of the heterotic string on symmetric orbifolds, especially in the context of classifications [7]. This language can be extended to Narain orbifolds naturally. The generalized space group $\mathbf{S}$ associated to a Narain orbifold is defined as being generated by the elements

$$
(\mathbb{1}, L) \text { and }\left(\Theta_{\alpha}, V_{\alpha}\right) \text { for all } L \in \mathbb{\Gamma} \text { and } \Theta_{\alpha} \in \mathbf{P} \text {, }
$$

where $V_{\alpha}$, a vector with $2 D+16$ components, is the so-called generalized shift which is associated to the twist $\Theta_{\alpha}$. Conversely, we demand that for all space group elements of the form $\left(\mathbb{1}, L^{\prime}\right) \in \mathbf{S}$ it follows that $L^{\prime} \in \mathbb{\Gamma}$. So, the Narain lattice $\mathbb{\Gamma}$ is the subgroup of $\mathbf{S}$ that 
contains all pure translations of $\mathbf{S}$. Note that a generator $\left(\Theta_{\alpha}, V_{\alpha}\right)$ is a generalized rototranslation if $V_{\alpha} \neq 0$, see [7]. These generators build the so-called Narain orbifold group $\mathbf{O}$, which is defined modulo lattice translations. Hence, just as $\mathbf{P}$, the Narain orbifold group $\mathbf{O}$ is a finite group.

A general space group element $g=(\Theta, \lambda) \in \mathbf{S}$ is defined to act on $Y$ as

$$
Y \mapsto g[Y]=(\Theta, \lambda)[Y]=\Theta Y+\lambda .
$$

Consequently, the unit element of $\mathbf{S}$ is given by

$$
(\mathbb{1}, 0) \in \mathbf{S} .
$$

The inverse element $g^{-1}$ of $g=(\Theta, \lambda) \in \mathbf{S}$ reads

$$
g^{-1}=\left(\Theta^{-1},-\Theta^{-1} \lambda\right) \in \mathbf{S} .
$$

Furthermore, two elements $g=(\Theta, \lambda)$ and $g^{\prime}=\left(\Theta^{\prime}, \lambda^{\prime}\right)$ are multiplied as

$$
g g^{\prime}=(\Theta, \lambda)\left(\Theta^{\prime}, \lambda^{\prime}\right)=\left(\Theta \Theta^{\prime}, \Theta \lambda^{\prime}+\lambda\right) \in \mathbf{S} .
$$

Hence, the generalized space group $\mathbf{S}$ is in general non-Abelian even if the Narain point group $\mathbf{P}$ is Abelian.

For orbifolds, each sector of string states is characterized by a boundary condition (4.2) and, thus, by the so-called constructing element $g=(\Theta, \lambda) \in \mathbf{S}$, where $\lambda=V_{\Theta}+L$ and $L \in \mathbb{\Gamma}$. Only those elements $g^{\prime} \in \mathbf{S}$ that commute with the constructing element $g$ yield projections and, hence, give rise to non-vanishing contributions to the twisted sector partition function. This only happens when

$$
\Theta \Theta^{\prime}=\Theta^{\prime} \Theta \text { and }(\mathbb{1}-\Theta) \lambda^{\prime}=\left(\mathbb{1}-\Theta^{\prime}\right) \lambda .
$$

\subsection{Conditions on the twists $\Theta_{\alpha}$}

Furthermore, we choose $L \in \mathbb{\Gamma}$ and consider

$$
\left(\Theta_{\alpha}, V_{\alpha}\right)(\mathbb{1}, L)\left(\Theta_{\alpha}, V_{\alpha}\right)^{-1}=\left(\mathbb{1}, \Theta_{\alpha} L\right) \stackrel{!}{\in} \mathbf{S} \Rightarrow \Theta_{\alpha} L \stackrel{!}{\in} \mathbb{\Gamma} .
$$

Thus, the lattice $\mathbb{\Gamma}$ is a normal subgroup of $\mathbf{S}$ and the Narain point group $\mathbf{P}$ has to consist of automorphisms of the Narain lattice, i.e.

$$
\Theta_{\alpha} \Gamma=\mathbb{\Gamma} .
$$

In addition, we have to impose eq. (4.4) on the twist generators $\Theta_{\alpha}$.

It is is interesting to pause here and reflect on the possible orders of twists for a given number of dimensions $D_{\Gamma}$ for general orbifolds associated to a lattice $\Gamma$. As is wellknown [87], if the order $K$ satisfies

$$
\phi(K) \leq D_{\Gamma},
$$

then there exists at least one lattice $\Gamma$ with rotational symmetry of order $K$. Here, $\phi(K)$ is the Euler $\phi$-function and this bound does not take into account that one can build point groups as direct sums of lower dimensional cases. However, in the current paper we are not working with a general lattice $\Gamma$ in $D_{\Gamma}$ dimensions, but with Narain lattices $\Gamma=\mathbb{\Gamma}$ with $D_{\mathbb{\Gamma}}=2 D+16$. Hence, contrary to the Euclidean case, it is not guaranteed that there exists a Narain lattice for each order $K$ satisfying the bound (4.14). 


\subsection{Orbifold projections of $\mathbf{I}$}

In general, a twist $\Theta \in \mathbf{P}$ of order $K$ acts as the identity in some directions of $Y$ while it acts as a $\mathbb{Z}_{K}$ twist on others. To identify these directions, we define projection operators for each twist $\Theta \in \mathbf{P}$ : the projection operators $\mathcal{P}_{\|}^{\Theta}$ and $\mathcal{P}_{\perp}^{\Theta}$ project a vector onto the directions in which $\Theta$ acts trivially and non-trivially, respectively. In detail, we define the projectors

$$
\mathcal{P}_{\|}^{\Theta}=\frac{1}{K} \sum_{j=0}^{K-1} \Theta^{j} \quad \text { and } \quad \mathcal{P}_{\perp}^{\Theta}=\mathbb{1}-\mathcal{P}_{\|}^{\Theta},
$$

with the properties

$$
\left(\mathcal{P}_{\|}^{\Theta}\right)^{2}=\mathcal{P}_{\|}^{\Theta}, \quad\left(\mathcal{P}_{\perp}^{\Theta}\right)^{2}=\mathcal{P}_{\perp}^{\Theta}, \quad \Theta \mathcal{P}_{\|}^{\Theta}=\mathcal{P}_{\|}^{\Theta}, \quad \mathcal{P}_{\perp}^{\Theta} \mathcal{P}_{\|}^{\Theta}=\mathcal{P}_{\|}^{\Theta} \mathcal{P}_{\perp}^{\Theta}=0
$$

Then, any vector $\lambda \in \mathbb{R}^{2 D+16}$ can be decomposed into two vectors $\lambda_{\|}^{\Theta}$ and $\lambda_{\perp}^{\Theta}$ according to

$$
\lambda_{\|}^{\Theta}=\mathcal{P}_{\|}^{\Theta} \lambda, \quad \lambda_{\perp}^{\Theta}=\mathcal{P}_{\perp}^{\Theta} \lambda \text { so that } \lambda=\lambda_{\|}^{\Theta}+\lambda_{\perp}^{\Theta},
$$

and $\Theta \lambda_{\|}^{\Theta}=\lambda_{\|}^{\Theta}$. The final relation clarifies the use of the subscript $\|$ : it defines the directions which are left invariant by $\Theta$.

Moreover, it is important to realize that the projected Narain lattice $\mathbb{\Gamma}_{\|}^{\Theta}=\mathcal{P}_{\|}^{\ominus} \mathbb{\Gamma}$ is in general not Narain. In detail, even if $\mathbb{\Gamma}$ and $\Theta \mathbb{\Gamma}$ are Narain lattices, see eq. (4.13), the normalisation $1 / K$ in the projection operator $\mathcal{P}_{\|}^{\Theta}$ in eq. (4.15) can make $\mathbb{\Gamma}_{\|}^{\Theta}$ non-Narain. A Narain lattice is said to be factorized w.r.t. the orbifold twists when

$$
\mathbb{\Gamma}_{\|}^{\ominus} \subset \mathbb{\Gamma}
$$

for all twists $\Theta \in \mathbf{P}$. In this case, obviously, all projected Narain lattices are themselves Narain again.

\subsection{Quantization of the generalized shifts $V_{\alpha}$}

For each Narain point group generator $\Theta_{\alpha}$ of order $K_{\alpha}$ we consider the generator $\left(\Theta_{\alpha}, V_{\alpha}\right)$ of the generalized space group $\mathbf{S}$. Then, its $K_{\alpha}$-th power reads

$$
\left(\Theta_{\alpha}, V_{\alpha}\right)^{K_{\alpha}}=\left(\Theta^{K_{\alpha}}, \sum_{j=0}^{K_{\alpha}-1} \Theta_{\alpha}^{j} V_{\alpha}\right)=\left(\mathbb{1}, K_{\alpha} \mathcal{P}_{\|}^{\alpha} V_{\alpha}\right) \stackrel{!}{\epsilon} \mathbf{S},
$$

(where $\mathcal{P}_{\|}^{\alpha}=\mathcal{P}_{\|}^{\Theta_{\alpha}}$ ) without summation over $\alpha$. Consequently, we have to demand the condition

$$
K_{\alpha} V_{\alpha_{\|}}=K_{\alpha} \mathcal{P}_{\|}^{\alpha} V_{\alpha}=\sum_{j=0}^{K_{\alpha}-1} \Theta_{\alpha}^{j} V_{\alpha}=L_{\alpha} \stackrel{!}{\epsilon} \mathbb{\Gamma} .
$$

That is, the shift $V_{\alpha}$ needs to be quantized in units of $K_{\alpha}$ in the directions where $\Theta_{\alpha}$ acts trivially, i.e. $V_{\alpha}$ is given by

$$
V_{\alpha}=\frac{L_{\alpha}}{K_{\alpha}}+\lambda_{\alpha} \quad \text { with } \quad \Theta_{\alpha} L_{\alpha}=L_{\alpha}, \mathcal{P}_{\|}^{\alpha} \lambda_{\alpha}=0 \quad \text { and } \quad L_{\alpha} \in \mathbb{\Gamma} .
$$


The same procedure can be applied to some arbitrary element $\Theta \in \mathbf{P}$ of order $K$ with associated element $\left(\Theta, V_{\Theta}\right) \in \mathbf{S}$. This yields

$$
V_{\Theta}=\frac{L_{\Theta}}{K}+\lambda_{\Theta} \quad \text { with } \quad \Theta L_{\Theta}=L_{\Theta}, \mathcal{P}_{\|}^{\Theta} \lambda_{\Theta}=0 \quad \text { and } \quad L_{\Theta} \in \mathbb{\Gamma} .
$$

As a remark, for example in the case when $\Theta_{\alpha}^{k}$ has a fixed torus for $0<k<K_{\alpha}$ (i.e. when $\Theta_{\alpha}^{k}$ has more invariant directions than $\Theta_{\alpha}$ ) eq. (4.22) gives stronger quantization conditions on the shift $V_{\alpha}$ than eq. (4.21).

Various choices for $V_{\alpha}$ correspond to the same Narain orbifold. Indeed, one can shift the origin, i.e.

$$
Y(\sigma, \bar{\sigma}) \mapsto Y(\sigma, \bar{\sigma})+Y_{0}
$$

and hence transform the generalized shifts $V_{\Theta} \mapsto V_{\Theta}-(\mathbb{1}-\Theta) Y_{0}$ for $Y_{0} \in \mathbb{R}^{2 D+16}$. (In light of the equivalence (2.6), only the (lower) $D+16$ components of $Y_{0}$ actually modify the description.) By doing so, one can set the components of $\lambda_{\Theta}$ either to zero or to some quantized value for each element $\left(\Theta, V_{\Theta}+\lambda_{\Theta}\right)$ of the Narain orbifold group $\mathbf{O}$. Especially, if the Narain point group is isomorphic to $\mathbb{Z}_{K}$ (with one generator $\Theta$ of order $K$ ) the generalized shift can be chosen as $V_{\Theta}=L_{\Theta} / K$ with $\Theta L_{\Theta}=L_{\Theta} \in \mathbb{\Gamma}$ without loss of generality.

\subsection{Preserving at least $\mathcal{N}=1$ target-space supersymmetry}

To enable the discussion on target-space supersymmetry we first need to recall a few facts about supersymmetry on the worldsheet. By construction the heterotic string has $(1,0)$ worldsheet supersymmetry. Hence, we can identify the worldsheet supercurrent

$$
T_{\mathrm{F}}=\psi_{\mathrm{R}}^{\mu} \bar{\partial} x_{\mu}+\psi_{\mathrm{R}}^{T} u_{\mathrm{Rr}} \bar{\partial} y_{\mathrm{r}}
$$

where $\psi_{\mathrm{R}}=\left(\psi_{\mathrm{R}}^{i}\right)$ are the real worldsheet fermions of the $D$ compactified dimensions and $u_{\mathrm{Rr}}$ is a $D \times D$ matrix. For each twist $\Theta_{\alpha}$, the space group action (4.7) is defined to be accompanied by a transformation of $\psi_{\mathrm{R}}$ as

$$
\psi_{\mathrm{R}} \mapsto g\left[\psi_{\mathrm{R}}\right]=\theta_{\alpha \mathrm{R}} \psi_{\mathrm{R}}
$$

where $\theta_{\alpha \mathrm{R}} \in \mathrm{O}(D ; \mathbb{R})$. Since the first term $\psi_{\mathrm{R}}^{\mu} \bar{\partial} x_{\mu}$ in eq. (4.24) is orbifold invariant the worldsheet supercurrent $T_{\mathrm{F}}$ has to be orbifold invariant as well. Consequently, we need to require that the twists on the right-moving coordinates $y_{\mathrm{r}}$ and on the right-moving fermions $\psi_{\mathrm{R}}$ are identified: $\theta_{\alpha \mathrm{R}}=u_{\mathrm{Rr}} \theta_{\alpha \mathrm{r}} u_{\mathrm{Rr}}^{-1}$.

Given that the properties of target-space fermions are determined by the right-moving momentum $p_{\mathrm{R}}$ associated to these right-moving fermions, as given eq. (2.12), the question of target-space supersymmetry is only affected by the transformations generated by $\theta_{\alpha} \mathrm{R}$ in the right-moving sector. In particular, target-space supersymmetry is independent of the choice one makes for $\Theta_{\alpha \mathrm{L}}$. Only if one restricts oneself to symmetric orbifolds, for which $\theta_{\alpha \mathrm{L}}=\theta_{\alpha \mathrm{l}} \oplus \mathbb{1}_{16}$ and $\theta_{\alpha \mathrm{l}}=\theta_{\alpha \mathrm{r}}=\theta_{\alpha \mathrm{R}}$ with $u_{\mathrm{Rr}}=\mathbb{1}_{D}$, see eq. (4.5), this connection is made.

Consequently, in order to preserve at least $\mathcal{N}=1$ supersymmetry in the $d$-dimensional target-space, the generators $\theta_{\alpha \mathrm{R}} \in \mathrm{SO}(D ; \mathbb{R})$ have to lie inside the appropriate special holonomy subgroup of $\mathrm{SO}(D ; \mathbb{R})$. For $D=4,6,7,8$ these subgroups are $\mathrm{SU}(2), \mathrm{SU}(3), \mathrm{G}_{2}$ 
and $\operatorname{Spin}(7)$, respectively, see e.g. [88]. For example, assume $D=6$ and an Abelian Narain point group, i.e.

$$
\mathbf{P} \cong \mathbb{Z}_{K_{1}} \times \ldots \mathbb{Z}_{K_{N_{\mathbf{P}}}}
$$

Then, the four-dimensional effective low energy theory possesses at least $\mathcal{N}=1$ supersymmetry if

$$
\phi_{\alpha \mathrm{R}}^{m}=0, \quad K_{\alpha} \phi_{\alpha \mathrm{R}}^{a} \equiv 0, \quad \frac{1}{2} \sum_{a} \phi_{\alpha \mathrm{R}}^{a}=0 .
$$

Here, we introduced the so-called twist vector $\phi_{\alpha \mathrm{R}}=\left(\phi_{\alpha \mathrm{R}}^{m}, \phi_{\alpha \mathrm{R}}^{a}\right)$ as the vector of phases corresponding to $\theta_{\alpha \mathrm{R}}$, such that $\theta_{\alpha \mathrm{R}}$ acts as

$$
\psi_{\mathrm{R}}^{m} \mapsto e^{2 \pi i \phi_{\alpha \mathrm{R}}^{m}} \psi_{\mathrm{R}}^{m}, \quad \psi_{\mathrm{R}}^{a} \mapsto e^{2 \pi i \phi_{\alpha \mathrm{R}}^{a}} \psi_{\mathrm{R}}^{a},
$$

using the complex indices defined below eq. (2.12). In fact, the last condition of eq. (4.27) only needs to be imposed mod integers (i.e. $\equiv)$ and this specific choice fixes the unbroken supercharges for $d=4$ and $\phi_{\alpha \mathrm{R}}^{a} \neq 0$ to be represented as $\pm\left(\frac{1}{2}, \frac{1}{2}, \frac{1}{2}, \frac{1}{2}\right)$.

\section{Moduli stabilization in Narain orbifolds}

As we have seen in the previous section, the space group description of Narain orbifolds is naturally formulated using the twist $\Theta$ and the generalized vielbein $E$. On the other hand, the question about moduli stabilization and classification, in particular, are more conveniently discussed in the so-called lattice basis in which the twist is encoded by an integral matrix $\widehat{\rho}$. Therefore, we begin this section with a discussion of Narain orbifolds in the lattice basis. Beside the integral twist matrices $\widehat{\rho}$, we introduce the generalized metric $\mathcal{H}$ and a closely related $\mathbb{Z}_{2}$-grading $\mathcal{Z}$. After that we investigate under which conditions Narain orbifolds exist and derive restrictions on the Narain moduli that have to be imposed in order to be compatible with the orbifold action. In particular, we derive a character formula that counts the dimension of the orbifold Narain moduli space.

\subsection{Narain orbifolds in the lattice basis}

Twists and shifts in the lattice basis. We have seen in eq. (4.13) that each point group generator $\Theta_{\alpha}$ has to map a Narain vector $E N$ to another Narain vector $E N^{\prime}=\Theta_{\alpha} E N$, see eq. (2.16). It follows that $N^{\prime}=\widehat{\rho}_{\alpha} N$, where we define $\widehat{\rho}_{\alpha}$ as

$$
\widehat{\rho}_{\alpha}=E^{-1} \Theta_{\alpha} E=\widehat{E}^{-1} \widehat{\Theta}_{\alpha} \widehat{E} \in \mathrm{GL}(2 D+16 ; \mathbb{Z}) .
$$

Here, we used $E=U R \widehat{E}$ and we absorbed $U$ in the definition of $\widehat{\Theta}_{\alpha}=R^{-1} U^{-1} \Theta_{\alpha} U R$.

The matrices $\widehat{\rho}_{\alpha}$ represent the generating twists $\Theta_{\alpha}$ in the so-called lattice basis. They have to be invertible over the integers (i.e. $\widehat{\rho}_{\alpha} \in \mathrm{GL}(2 D+16 ; \mathbb{Z})$ ) because each $\widehat{\rho}_{\alpha}$ has to map an integer vector $N$ one-to-one to another integer vector $N^{\prime}$. Furthermore, they inherit the following conditions

$$
\widehat{\rho}_{\alpha}^{T} \widehat{\eta} \widehat{\rho}_{\alpha}=\widehat{\eta} \quad \text { and } \quad \hat{\rho}_{\alpha}^{K_{\alpha}}=\mathbb{1}
$$


since the generating twists $\Theta_{\alpha}$ are elements of $\mathrm{O}_{\eta}(D, D+16 ; \mathbb{R})$ of finite order $K_{\alpha}$. The integral matrices $\widehat{\rho}_{\alpha}$ generate the so-called Narain point group in the lattice basis $\widehat{\mathbf{P}} \subset \mathrm{O}_{\widehat{\eta}}(D, D+16 ; \mathbb{Z})$, while twists $\Theta_{\alpha} \in \mathbf{P}$ are given in the so-called coordinate basis. The lattice basis will be of special importance for the classification of Narain orbifolds later in section 6.1. Moreover, the space group generators $\left(\Theta_{\alpha}, V_{\alpha}\right)$ and $(\mathbb{1}, L) \in \mathbf{S}$ can be represented in the lattice basis as

$$
\left(\widehat{\rho}_{\alpha}, \frac{1}{K_{\alpha}} N_{\alpha}\right) \in \widehat{\mathbf{S}} \text { and }(\mathbb{1}, N) \in \widehat{\mathbf{S}}
$$

where $V_{\alpha}=\frac{1}{K_{\alpha}} E N_{\alpha}$ and $L=E N$ for $N, N_{\alpha} \in \mathbb{Z}^{2 D+16}$.

Generalized metric. Eq. (5.2) represents two out of the three properties (4.4) of the generators $\Theta_{\alpha}$ in the lattice basis. The remaining one, $\Theta_{\alpha}^{T} \Theta_{\alpha}=\mathbb{1}$, can be cast in the form

$$
\widehat{\rho}_{\alpha}^{T} \mathcal{H} \widehat{\rho}_{\alpha}=\mathcal{H}
$$

where we have introduced the so-called generalized metric $\mathcal{H}$ defined as

$$
\mathcal{H}=E^{T} E=\widehat{E}^{T}(e, B, A) R^{T} R \widehat{E}(e, B, A) .
$$

In other words, condition (5.4) states that the generators $\widehat{\rho}_{\alpha}$ and the generalized metric $\mathcal{H}$ have to be compatible.

The generalized metric is given explicitly by

$$
\mathcal{H}(G, B, A)=\left(\begin{array}{ccc}
G+A^{T} A+C G^{-1} C^{T} & -C G^{-1} & \left(\mathbb{1}_{D}+C G^{-1}\right) A^{T} \alpha_{\mathrm{g}} \\
-G^{-1} C^{T} & G^{-1} & -G^{-1} A^{T} \alpha_{\mathrm{g}} \\
\alpha_{\mathrm{g}}^{T} A\left(\mathbb{1}_{D}+G^{-1} C^{T}\right) & -\alpha_{\mathrm{g}}^{T} A G^{-1} & \alpha_{\mathrm{g}}^{T}\left(\mathbb{1}_{16}+A G^{-1} A^{T}\right) \alpha_{\mathrm{g}}
\end{array}\right),
$$

using eqs. (2.26) and (2.39). It is an interesting object in its own right: assume one is given a generic Narain lattice (with moduli-independent Narain metric $\widehat{\eta}=E^{T} \eta E$ as given in eq. (2.23)) by specifying the generalized vielbein $E$, then it might be rather awkward to determine the matrix $U$ from $E=U R \widehat{E}$ such that we can read off the moduli contained in the matrix $\widehat{E}$. As the generalized metric $\mathcal{H}$ is independent of $U$, it can be used to read off the metric $G$ of the $D$-dimensional torus, the $B$-field and the Wilson line matrix $A$. As the explicit expression of the generalized metric (5.6) shows, not all its components are independent, i.e. $\mathcal{H}$ is not a generic $(2 D+16) \times(2 D+16)$ matrix. Indeed, $\mathcal{H}$ satisfies the constraints

$$
\mathcal{H} \widehat{\eta}^{-1} \mathcal{H}=\widehat{\eta} \quad \text { and } \quad \mathcal{H}^{T}=\mathcal{H},
$$

as follows from its definition (5.5) .

A $\mathbb{Z}_{\mathbf{2}}$ grading. The compatibility condition (5.4) of the orbifold twists in the lattice basis can also be represented as

$$
\mathcal{Z} \widehat{\rho}_{\alpha}=\widehat{\rho}_{\alpha} \mathcal{Z}
$$

where we have defined

$$
\mathcal{Z}=\widehat{\eta}^{-1} \mathcal{H}=E^{-1} \eta E=\widehat{E}(e, B, A)^{-1} \widehat{I} \widehat{E}(e, B, A) \in \mathrm{O}_{\widehat{\eta}}(D, D+16, \mathbb{R}) .
$$


The second expression in this equation is obtained using $\widehat{I}=R^{-1} \eta R$, as given in table 1 , and the relation $R^{T} R=\widehat{\eta} \widehat{I}$. Explicitly, $\mathcal{Z}$ is given by

$$
\mathcal{Z}(G, B, A)=\left(\begin{array}{ccc}
-G^{-1} C^{T} & G^{-1} & -G^{-1} A^{T} \alpha_{\mathrm{g}} \\
G+A^{T} A+C G^{-1} C^{T} & -C G^{-1} & \left(\mathbb{1}_{D}+C G^{-1}\right) A^{T} \alpha_{\mathrm{g}} \\
\alpha_{\mathrm{g}}^{-1} A\left(\mathbb{1}_{D}+G^{-1} C^{T}\right) & -\alpha_{\mathrm{g}}^{-1} A G^{-1} & \alpha_{\mathrm{g}}^{-1}\left(\mathbb{1}_{16}+A G^{-1} A^{T}\right) \alpha_{\mathrm{g}}
\end{array}\right) .
$$

The constraints (5.7), which the generalized metric satisfies, translate to the following conditions on $\mathcal{Z}$ :

$$
\mathcal{Z}^{T} \widehat{\eta} \mathcal{Z}=\widehat{\eta} \quad \text { and } \quad \mathcal{Z}^{2}=\mathbb{1} .
$$

This can be confirmed by using eq. (5.7) and the fact that $\widehat{I}^{2}=\mathbb{1}$. Given its definition (5.9), the matrix $\mathcal{Z}$ has signature $(D, D+16)$, just as $\eta$ (and $\widehat{I}$ ). This leads to a grading of the Narain lattice: it characterizes the distinction between $D$ right- and $D+16$ left-moving directions of the Narain lattice.

\subsection{On the existence of Narain orbifolds for a given point group}

Assume a given finite point group $\widehat{\mathbf{P}} \subset \mathrm{O}_{\widehat{\eta}}(D, D+16 ; \mathbb{Z})$ with generators $\widehat{\rho}_{\alpha}$ in the lattice basis. We want to understand these generators $\widehat{\rho}_{\alpha}$ as the crucial ingredient in the definition of a Narain orbifold. Therefore, we have to address the following question: under which condition does a corresponding Narain orbifold exist? In terms of the terminology introduced in section 4 this can be phrased as follows: when does a Narain lattice exist, such that all generators $\Theta_{\alpha}$ of the corresponding group $\mathbf{P}$ in the coordinate basis satisfy (4.4) and are symmetries of this lattice (4.13)?

In the following, we will answer this question in the lattice basis. Then, the conditions on $\Theta_{\alpha}$ translate to conditions (5.2) and (5.4) on $\widehat{\rho}_{\alpha} \in \widehat{\mathbf{P}}$. In fact, eq. (5.2) is fulfilled by assumption (i.e $\widehat{\mathbf{P}} \subset \mathrm{O}_{\widehat{\eta}}(D, D+16 ; \mathbb{Z})$ and finite). Thus, it remains to show that eq. (5.4) is fulfilled, i.e. we have to find a generalized metric that is compatible with all generators $\widehat{\rho}_{\alpha}$. Consequently, a Narain orbifold with given point group $\widehat{\mathbf{P}}$ exists if one finds Narain moduli $G, B$ and $A$ that are invariant under $\widehat{\rho}_{\alpha} \in \widehat{\mathbf{P}}$.

If such a generalized vielbein exists, then generically, not all the moduli of the Narain torus compactification are still free; some Narain moduli are stabilized. Thus, we can use our discussion on the transformation properties of Narain moduli under general $T$-duality transformations in section 3.3 in order to derive conditions for moduli stabilization.

To address these questions, we study the existence of both a twist $\Theta_{\alpha} \in \mathrm{O}(D ; \mathbb{R}) \times \mathrm{O}(D+16 ; \mathbb{R})$ for each $\hat{\rho}_{\alpha}$ and a compatible generalized vielbein $\widehat{E}(e, B, A)$, i.e.

$$
\Theta_{\alpha} R \widehat{E}(e, B, A)=R \widehat{E}(e, B, A) \widehat{\rho}_{\alpha},
$$

which is equivalent to eq. (5.1) by absorbing $U$ in the definition of $\Theta_{\alpha}$. Eq. (5.12) constitutes nine coupled matrix equations for the $D(D+16)$ Narain moduli $G, B, A$ and the $D(D-1) / 2$ and $(D+16)(D+15) / 2$ parameters inside each of the generators $\Theta_{\alpha}$.

Instead of trying to solve all nine coupled matrix equations, we first focus on a subset of only three matrix equations

$$
W \widehat{\rho}_{\alpha}=\theta_{\alpha \mathrm{r}} W \quad \text { with } \quad W=\sqrt{2}(R \widehat{E})_{\mathrm{r}}=e^{-T}\left(G+C^{T},-\mathbb{1}_{D}, A^{T} \alpha_{\mathrm{g}}\right)
$$


(where $\theta_{\alpha \mathrm{r}}$ is the $u_{\mathrm{r}}$ part of the matrix $\Theta_{\alpha}$ as defined in eq. (2.38)) that determine the Narain moduli uniquely already. Expanding out eq. (5.13), we obtain

$$
\begin{aligned}
& -\left(\widehat{\rho}_{\alpha}\right)_{21}+\left(G+C^{T}\right)\left(\widehat{\rho}_{\alpha}\right)_{11}+A^{T} \alpha_{\mathrm{g}}\left(\widehat{\rho}_{\alpha}\right)_{31}=\rho_{\alpha \mathrm{r}}^{-T}\left(G+C^{T}\right) \\
& -\left(\widehat{\rho}_{\alpha}\right)_{22}+\left(G+C^{T}\right)\left(\widehat{\rho}_{\alpha}\right)_{12}+A^{T} \alpha_{\mathrm{g}}\left(\widehat{\rho}_{\alpha}\right)_{32}=-\rho_{\alpha \mathrm{r}}^{-T} \\
& -\left(\widehat{\rho}_{\alpha}\right)_{23}+\left(G+C^{T}\right)\left(\widehat{\rho}_{\alpha}\right)_{13}+A^{T} \alpha_{\mathrm{g}}\left(\widehat{\rho}_{\alpha}\right)_{33}=\rho_{\alpha \mathrm{r}}^{-T} A^{T} \alpha_{\mathrm{g}}
\end{aligned}
$$

where $\rho_{\alpha \mathrm{r}}:=e^{-1} \theta_{\alpha \mathrm{r}} e$. (Note that there is a redundancy between $e$ and $\theta_{\alpha \mathrm{r}}$, which reflects the fact that the vielbein $e$ is not uniquely determined by the metric $G$.)

It is sufficient to solve only these three matrix equations (5.14) in order to find a solution of all nine equations (5.12) because of the coset decomposition (3.4): indeed, we can alternatively obtain the set of coupled equations (5.14) by comparing eq. (5.12) to eq. (3.4). They are identical if we determine each twist $\Theta_{\alpha}$ from eq. (3.10) using $U_{\widehat{M}}=\Theta_{\alpha}$ (hence, in particular $u_{\mathrm{r}}=\theta_{\alpha \mathrm{r}}$ ) and $\widehat{M}=\widehat{\rho}_{\alpha}$. Furthermore, we have to set $G^{\prime}=G, B^{\prime}=B$ and $A^{\prime}=A$, where the primed objects are determined by the transformation of the generalized metric

$$
\mathcal{H}\left(G^{\prime}, B^{\prime}, A^{\prime}\right)=\widehat{\rho}_{\alpha}^{T} \mathcal{H}(G, B, A) \widehat{\rho}_{\alpha} \stackrel{!}{=} \mathcal{H}(G, B, A),
$$

using eq. (5.5). Therefore, using eq. (3.11) the moduli of the Narain lattice are constrained according to

$$
\widehat{M}_{\alpha 1}^{T} \rho_{\alpha \mathrm{r}}=G+C, \quad \widehat{M}_{\alpha 2}^{T} \rho_{\alpha \mathrm{r}}=-\mathbb{1} \text { and } \widehat{M}_{\alpha 3}^{T} \rho_{\alpha \mathrm{r}}=\alpha_{\mathrm{g}}^{T} A,
$$

for each generator of the point group $\widehat{\rho}_{\alpha}$. Inserting the moduli-dependent short-hands $\widehat{M}_{\alpha i}$ from eq. (3.8) the resulting equations are again eqs. (5.14). In summary, for a given finite group $\widehat{\mathbf{P}} \subset \mathrm{O}_{\widehat{\eta}}(D, D+16 ; \mathbb{Z})$ there exists a Narain lattice such that $\widehat{\mathbf{P}}$ is a point group of this lattice if the Narain moduli can be chosen such that they are invariant under the orbifold action, i.e. $G^{\prime}=G, B^{\prime}=B$ and $A^{\prime}=A$, see section 5 .

Eq. (5.14b) can be used to constrain $\rho_{\alpha \mathrm{r}}$. Inserting this in the other two equations of eqs. (5.14) leads to two coupled quadratic matrix equations

$$
\begin{aligned}
& \left(G+C^{T}\right) \widehat{\rho}_{12}\left(G+C^{T}\right)+A^{T} \alpha_{\mathrm{g}}\left(\widehat{\rho}_{32}\left(G+C^{T}\right)+\widehat{\rho}_{31}\right) \\
& -\widehat{\rho}_{22}\left(G+C^{T}\right)+\left(G+C^{T}\right) \widehat{\rho}_{11}=\widehat{\rho}_{21}, \\
& A^{T} \alpha_{\mathrm{g}} \widehat{\rho}_{32} A^{T} \alpha_{\mathrm{g}}+\left(G+C^{T}\right)\left(\widehat{\rho}_{13}+\widehat{\rho}_{12} A^{T} \alpha_{\mathrm{g}}\right)-\widehat{\rho}_{22} A^{T} \alpha_{\mathrm{g}}+A^{T} \alpha_{\mathrm{g}} \widehat{\rho}_{33}=\widehat{\rho}_{23} .
\end{aligned}
$$

for each generator $\widehat{\rho}=\widehat{\rho}_{\alpha}$ of the point group $\widehat{\mathbf{P}}$. These conditions can be thought of as algebraic Riccati equations (see e.g. [89]) which constrain some and sometimes even all the moduli $G, B$ and $A$. Hence we have reduced the existence question of Narain orbifolds to the question whether these Riccati equations admit real solutions.

\subsection{Mapping from the lattice basis to the coordinate basis}

Assume we are given a finite point group $\widehat{\mathbf{P}} \subset \mathrm{O}_{\widehat{\eta}}(D, D+16$; $\mathbb{Z})$ with generators $\widehat{\rho}_{\alpha}$ in the lattice basis and we want to know a compatible Narain lattice as well as the twists $\Theta_{\alpha}$ in the coordinate basis. To obtain this data we can perform the following steps: first, we 
find a solution to eqs. (5.17), i.e. find orbifold invariant moduli $G, B$ and $A$. After that we make a choice for a geometrical vielbein $e$ such that $e^{T} e=G$. By doing so, we have obtained a generalized vielbein $E=R \widehat{E}(e, B, A)$, which is compatible with $\widehat{\mathbf{P}}$ in the sense of eq. (5.12). Finally, we compute the twists in the lattice basis: using the geometrical vielbein $e$ we can determine the right-moving twists $\theta_{\alpha \mathrm{r}}=e \rho_{\alpha \mathrm{r}} e^{-1}$, where $\rho_{\alpha \mathrm{r}}$ is given by eq. (5.14b). Consequently, we can compute the blocks of $\Theta_{\alpha}$ from eq. (3.10), i.e.

$$
\begin{aligned}
\theta_{\alpha \mathrm{l}} & =\left(\mathbb{1}_{D}-2 e\left(\widehat{\rho}_{\alpha}\right)_{12} \widehat{M}_{\alpha 2}^{-1} e^{T}\right) \theta_{\alpha \mathrm{r}} \\
\theta_{\alpha \mathrm{L}} & =\sqrt{2} e\left(\left(\widehat{\rho}_{\alpha}\right)_{13}-\left(\widehat{\rho}_{\alpha}\right)_{12} \widehat{M}_{\alpha 2}^{-1} \widehat{M}_{\alpha 3}\right) \alpha_{\mathrm{g}}^{-1}, \\
\theta_{\alpha \mathrm{Ll}} & =-\sqrt{2}\left(\alpha_{\mathrm{g}}\left(\widehat{\rho}_{\alpha}\right)_{32}+A\left(\widehat{\rho}_{\alpha}\right)_{12}\right) \widehat{M}_{\alpha 2}^{-1} e^{T} \theta_{\alpha \mathrm{r}}, \\
\theta_{\alpha \mathrm{L}} & =A\left(\widehat{\rho}_{\alpha}\right)_{13} \alpha_{\mathrm{g}}^{-1}+\alpha_{\mathrm{g}}\left(\widehat{\rho}_{\alpha}\right)_{33} \alpha_{\mathrm{g}}^{-1}-\left(A\left(\widehat{\rho}_{\alpha}\right)_{12}+\alpha_{\mathrm{g}}\left(\widehat{\rho}_{\alpha}\right)_{32}\right) \widehat{M}_{\alpha 2}^{-1} \widehat{M}_{\alpha 3} \alpha_{\mathrm{g}}^{-1},
\end{aligned}
$$

where $\widehat{M}_{\alpha i}$ for $i=1,2,3$ are defined in eq. (3.8) setting $\widehat{M}=\widehat{\rho}_{\alpha}$. This method we will be exemplified in section 8 where we discuss a number of two-dimensional Narain orbifolds.

An important characterization of heterotic Narain orbifolds is whether they are symmetric or asymmetric. In section 4.1 we defined a Narain orbifold to be symmetric if there is a coordinate basis such that eq. (4.5) holds. In the lattice basis, a sufficient but not necessary condition for a Narain orbifold to be symmetric is $\left(\widehat{\rho}_{\alpha}\right)_{12}=0$ : first of all notice that $\left(\widehat{\rho}_{\alpha}\right)_{12}=0$ implies $\left(\widehat{\rho}_{\alpha}\right)_{13}=0$ and $\left(\widehat{\rho}_{\alpha}\right)_{32}=0$ since $\widehat{\rho}_{\alpha}^{T} \widehat{\eta} \widehat{\rho}_{\alpha}=\widehat{\eta}$. Consequently, the conditions (5.17) become linear in the moduli and, hence, not all Narain moduli are frozen. Furthermore, using eqs. (5.18) we obtain

$$
\theta_{\alpha \mathrm{l}}=\theta_{\alpha \mathrm{r}}, \quad \theta_{\alpha \mathrm{lL}}=\theta_{\alpha \mathrm{Ll}}=0, \quad \theta_{\alpha \mathrm{L}}=\alpha_{\mathrm{g}}\left(\widehat{\rho}_{\alpha}\right)_{33} \alpha_{\mathrm{g}}^{-1}
$$

Hence, any generator $\widehat{\rho}_{\alpha} \in \widehat{\mathbf{P}}$ with $\left(\widehat{\rho}_{\alpha}\right)_{12}=0$ and $\left(\widehat{\rho}_{\alpha}\right)_{33}=\mathbb{1}_{16}$ corresponds to a symmetric twist. However, the converse is in general not true. In section 8 we provide examples for both cases: in section 8.2 we list several Narain orbifolds that are necessarily symmetric because $\left(\widehat{\rho}_{\alpha}\right)_{12}=0$ and in section 8.4 we give one Narain orbifold that is symmetric even though $\left(\widehat{\rho}_{\alpha}\right)_{12} \neq 0$.

\subsection{Dimensionality of the Narain orbifold moduli space}

Assuming that a Narain orbifold exists, i.e. assuming that we have found a generalized vielbein $\widehat{E}_{0}$ that satisfies eq. (5.12), we want to determine the number of unconstrained Narain moduli. In other words, we want to count the number of moduli perturbations $\delta \mathcal{H}$ that can deform the associated generalized metric $\mathcal{H}_{0}$ such that $\mathcal{H}_{0}+\delta \mathcal{H}$ remains invariant under the Narain orbifold action.

To address this question, we make use of the results from appendix $\mathrm{A}$ and set $\widehat{\mathbf{H}}=\widehat{\mathbf{P}}$. Then, the tangent space to the orbifold-invariant moduli space is given by

$$
\mathcal{M}_{\widehat{\mathbf{P}}}=\left\{\delta \mathfrak{m}_{\widehat{\mathbf{P}}}=\mathcal{P}_{\widehat{\mathbf{P}}} \delta \mathfrak{m}\right\}
$$


where the projection operator $\mathcal{P}_{\widehat{\mathbf{P}}}$ is defined in eq. (A.11). The moduli deformations $\delta \mathcal{H}$, can be parametrized as follows

$$
\delta \mathcal{H}=E_{0}^{T} \delta \mathfrak{h} E_{0}, \quad \delta \mathfrak{h}=\left(\begin{array}{cc}
0 & \delta \mathfrak{m} \\
\delta \mathfrak{m}^{T} & 0
\end{array}\right) \quad \delta \mathfrak{m}=\left(e_{0}^{-T}\left(\delta G-\delta B^{\prime}\right) e_{0}^{-1}, \sqrt{2} e_{0}^{-T} \delta A^{T}\right),
$$

where $\delta B^{\prime}=\delta B+\frac{1}{2} \delta A^{T} A_{0}-\frac{1}{2} A_{0}^{T} \delta A, \delta G=\delta e^{T} e_{0}+e_{0}^{T} \delta e$.

According to eq. (A.13) the dimension of the orbifold-invariant Narain moduli space, i.e. the number of moduli, is determined by

$$
\operatorname{dim}\left(\mathcal{M}_{\widehat{\mathbf{P}}}\right)=\left\langle\chi_{\mathrm{r}}, \chi_{\mathrm{L}}\right\rangle=\frac{1}{|\mathbf{P}|} \sum_{\Theta \in \mathbf{P}} \chi_{\mathrm{r}}(\Theta) \chi_{\mathrm{L}}(\Theta)^{*},
$$

where we have introduce the right- and left-characters

$$
\chi_{\mathrm{r}}(\Theta)=\operatorname{tr}\left[\theta_{\mathrm{r}}\right]=\operatorname{tr}\left[\frac{\mathbb{1}-\eta}{2} \Theta\right], \quad \chi_{\mathrm{L}}(\Theta)=\operatorname{tr}\left[\Theta_{\mathrm{L}}\right]=\operatorname{tr}\left[\frac{\mathbb{1}+\eta}{2} \Theta\right]
$$

respectively. Because of this character formula (5.22), the number of moduli $\operatorname{dim}\left(\mathcal{M}_{\widehat{\mathbf{P}}}\right)$ for Narain orbifolds only depends on the representations of $\theta_{\mathrm{r}}$ and $\Theta_{\mathrm{L}}$ of the point group $\mathbf{P}$, but not on conjugation of $\Theta$ with $U \in \mathrm{O}(D ; \mathbb{R}) \times \mathrm{O}(D+16 ; \mathbb{R})$.

The number of fixed moduli is given by $D(D+16)-\operatorname{dim}\left(\mathcal{M}_{\widehat{\mathbf{P}}}\right)$. In particular, all Narain moduli are frozen if $\operatorname{dim}\left(\mathcal{M}_{\widehat{\mathbf{P}}}\right)=0$. In this case, the Narain orbifold moduli space $\mathcal{M}_{\widehat{\mathbf{P}}}$ is a point (or a set of disjoint points). This happens when the right- and leftcharacters (5.23) are orthogonal. In light of this, we can use the property that characters of irreducible representations form an orthonormal basis to analyze eq. (5.22). In detail, for two (complex) irreducible representations $\boldsymbol{\mu}$ and $\boldsymbol{\nu}$ of the finite point group $\mathbf{P}$ we have

$$
\left\langle\chi_{\boldsymbol{\mu}}, \chi_{\boldsymbol{\nu}}\right\rangle=\left\{\begin{array}{ll}
1 & \text { if } \boldsymbol{\mu}=\boldsymbol{\nu} \\
0 & \text { else }
\end{array} .\right.
$$

This can be used to construct some situations with all moduli fixed, i.e. $\operatorname{dim}\left(\mathcal{M}_{\widehat{\mathbf{H}}}\right)=0$ :

- If the matrix representations of $\theta_{\mathrm{r}}$ and $\Theta_{\mathrm{L}}$ are both irreducible, they have to be different, since the former is $D$-dimensional while the latter is $(D+16)$-dimensional, and hence, their characters are orthogonal.

- If the representations of $\theta_{\mathrm{r}}$ and $\Theta_{\mathrm{L}}$ are reducible, one can decompose them into irreducible ones as

$$
\theta_{\mathrm{r}}=\bigoplus_{\boldsymbol{\mu}} \theta_{\mathrm{r} \boldsymbol{\mu}}, \quad \Theta_{\mathrm{L}}=\bigoplus_{\boldsymbol{\nu}} \Theta_{\mathrm{L} \boldsymbol{\nu}}, \quad \Rightarrow \quad \chi_{\mathrm{r}}=\sum_{\boldsymbol{\mu}} \chi_{\mathrm{r} \boldsymbol{\mu}}, \quad \chi_{\mathrm{L}}=\sum_{\nu} \chi_{\mathrm{r} \boldsymbol{\nu}}
$$

where the irreducible representations $\theta_{\mathrm{r} \boldsymbol{\mu}}$ and $\Theta_{\mathrm{L} \boldsymbol{\nu}}$ are in general complex. Hence, if and only if $\theta_{\mathrm{r}}$ and $\Theta_{\mathrm{L}}$ do not contain any irreducible representation in common, again the characters $\chi_{\mathrm{r}}$ and $\chi_{\mathrm{L}}$ are orthogonal. An particular example of this is obtained, when $\Theta_{\mathrm{L}}=\mathbb{1}$ and $\theta_{\mathrm{r}}$ does not contain any trivial one-dimensional representations of $\mathbf{P}$. 


\subsection{A $T$-fold constructed as an asymmetric $\mathbb{Z}_{2}$ Narain orbifold}

To illustrate the various results, we conclude this section by considering a simple but instructive construction of a $T$-fold: we define an asymmetric $\mathbb{Z}_{2}$ Narain orbifold by choosing

$$
\widehat{\rho}=\widehat{I}=\left(\begin{array}{ccc}
0 & \mathbb{1}_{D} & 0 \\
\mathbb{1}_{D} & 0 & 0 \\
0 & 0 & \mathbb{1}_{16}
\end{array}\right)
$$

see table 1. First, we identify a specific example of a compatible Narain lattice using the $\mathbb{Z}_{2}$ grading $\mathcal{Z}$. Then, we will use the discussion from section 5.2 to see that this is actually the most general solution. Finally, we confirm this by counting the number of unstabilized Narain moduli using section 5.4.

To find a compatible Narain lattice, we notice that $\mathcal{Z}=\widehat{I}$ is a valid $\mathbb{Z}_{2}$ grading satisfying eq. (5.8). Hence, we can easily read off

$$
e=G=\mathbb{1}_{D}, \quad B=0 \text { and } A=0
$$

from eq. (5.10) as a possible choice for the Narain moduli. Alternatively, we can study the solutions of eqs. (5.17). In this case these equations read:

$$
\left(G+C^{T}\right)(G+C)=\mathbb{1}_{D}, \quad A^{T} \alpha_{\mathrm{g}}=0 .
$$

Again, it is not difficult to confirm that eqs. (5.27) constitute a solution.

Consequently, we find $\widehat{E}(e, B, A)=\mathbb{1}$ and we obtain the twist $\Theta$ in the coordinate basis from eq. (5.12) as

$$
\Theta=R \widehat{E}(e, B, A) \widehat{\rho} \widehat{E}(e, B, A)^{-1} R^{-1}=R \widehat{I} R^{-1}=\eta=\left(\begin{array}{ccc}
-\mathbb{1}_{D} & 0 & 0 \\
0 & \mathbb{1}_{D} & 0 \\
0 & 0 & \mathbb{1}_{16}
\end{array}\right) \text {, }
$$

i.e. $\theta_{\mathrm{r}}=-\mathbb{1}_{D}, \theta_{\mathrm{l}}=\mathbb{1}_{D}$ and $\theta_{\mathrm{L}}=\mathbb{1}_{16}$.

In fact, all Narain moduli are stabilized in this case as we are going to show next. We use eq. (3.8) with $\widehat{M}=\widehat{I}$, which yields

$$
\widehat{M}_{1}=-\mathbb{1}_{D} \quad, \quad \widehat{M}_{2}=G+C^{T} \text { and } \widehat{M}_{3}=A^{T} \alpha_{\mathrm{g}} .
$$

Then, the Narain moduli are subject to the constraints (5.16). In this example, they read

$$
\mathbb{1}_{D}=G+C \text { and } A=-A .
$$

using $\rho_{\mathrm{r}}=e^{-1} \theta_{\mathrm{r}} e=-\mathbb{1}_{D}$. Consequently, all Narain moduli are stabilized and their values are given by eqs. (5.27).

The fact that all Narain moduli are stabilized in this example is also easy to understand using the number of unstabilized Narain moduli $\operatorname{dim}\left(\mathcal{M}_{\widehat{\mathbf{P}}}\right)$, see eq. (5.22): $\theta_{\mathrm{r}}$ consists of $D$ non-trivial irreducible representations of the $\mathbb{Z}_{2}$ point group, while $\Theta_{\mathrm{L}}$ consists of $D+16$ trivial irreducible representations. As the characters of different irreducible representations are orthogonal, we easily find $\operatorname{dim}\left(\mathcal{M}_{\widehat{\mathbf{P}}}\right)=0$. 


\section{Towards a classification of Narain orbifolds}

In this section we would like to lay the foundations for a classification of inequivalent Narain orbifolds. In general, the key to a classification of any structure is to identify those transformations that relate (or even define) equivalent structures. These transformations can be used to define equivalence relations that consequently give rise to equivalence classes. For the classification of $D$-dimensional - geometrical - orbifolds the structure turns out to be the space group and the equivalence relations are based on the notions of $\mathbb{Q}-, \mathbb{Z}$ - and affine-classes [7]. In this section we show that extending these notions to generalized space groups is the key for a classification of Narain orbifolds.

In more detail, for the classification of Narain orbifolds we identify three main structures: (i) the integral Narain point group $\widehat{\mathbf{P}}$ of finite lattice automorphisms, (ii) an associated Narain lattice $\mathbb{\Gamma}$ (given by a geometrical torus with metric $G$, a $B$-field and Wilson lines $A$ ) that is compatible with the point group and, finally, (iii) the full generalized space group $\mathbf{S}$, which fully specifies a Narain orbifold as we have seen in section 4. The main purposes of this section are to define equivalences for these three structures, namely Narain $\mathbb{Q}-, \mathbb{Z}$ - and Poincaré-equivalences, together with their associated equivalence-classes and to analyze their interpretations.

\subsection{Narain $\mathbb{Q}$ - and $\mathbb{Z}$-classes}

For the definition of Narain $\mathbb{Q}$ - and $\mathbb{Z}$-classes we need to describe the Narain point group in the lattice basis, where $\widehat{\mathbf{P}} \subset \mathrm{O}_{\widehat{\eta}}(D, D+16 ; \mathbb{Z})$, see section 5.1. Then, one only has to consider integral finite order elements $\widehat{\rho}_{\alpha} \in \widehat{\mathbf{P}}$. Since Narain $\mathbb{Q}$ - and $\mathbb{Z}$-classes are analogously defined, we take the field $\mathbb{F}$ to be either $\mathbb{Q}$ - and $\mathbb{Z}$ and begin with the definition of $\mathbb{F}$-equivalence: two matrices $\widehat{\rho} \in \mathrm{O}_{\widehat{\eta}}(D, D+16 ; \mathbb{Z})$ and $\widehat{\rho}^{\prime} \in \mathrm{O}_{\widehat{\eta}^{\prime}}(D, D+16 ; \mathbb{Z})$ are defined to be $\mathbb{F}$-equivalent if there exists a matrix $\widehat{M} \in \mathrm{GL}(2 D+16 ; \mathbb{F})$ such that

$$
\widehat{\rho}^{\prime}=\widehat{M}^{-1} \widehat{\rho} \widehat{M} \text { and } \widehat{\eta}^{\prime}=\widehat{M}^{T} \widehat{\eta} \widehat{M} .
$$

Two Narain points groups $\widehat{\mathbf{P}} \subset \mathrm{O}_{\widehat{\eta}}(D, D+16 ; \mathbb{Z})$ and $\widehat{\mathbf{P}}^{\prime} \subset \mathrm{O}_{\widehat{\eta}^{\prime}}(D, D+16 ; \mathbb{Z})$ are said to be $\mathbb{F}$-equivalent if there exists a single matrix $\widehat{M} \in \mathrm{GL}(2 D+16 ; \mathbb{F})$ such that

$$
\widehat{\mathbf{P}}^{\prime}=\widehat{M}^{-1} \widehat{\mathbf{P}} \widehat{M} \text { and } \widehat{\eta}^{\prime}=\widehat{M}^{T} \widehat{\eta} \widehat{M} \text {. }
$$

Note that if two point groups are from the same $\mathbb{Z}$-class they are also from the same $\mathbb{Q}$ class, because if $\widehat{M} \in \mathrm{GL}(2 D+16 ; \mathbb{Z})$ then $\widehat{M} \in \mathrm{GL}(2 D+16 ; \mathbb{Q})$. But the converse is not true, i.e. two point groups from the same $\mathbb{Q}$-class can be in inequivalent $\mathbb{Z}$-classes.

\subsection{Interpretation of Narain $\mathbb{Q}$ - and $\mathbb{Z}$-classes}

To prepare the interpretation of the Narain $\mathbb{Q}$ - and $\mathbb{Z}$-classes, let us assume that two Narain point groups $\widehat{\mathbf{P}}$ and $\widehat{\mathbf{P}}^{\prime}$ are from the same $\mathbb{F}$-class, where the field $\mathbb{F}$ is either $\mathbb{Q}$ or $\mathbb{Z}$. Then, there exists a matrix $\widehat{M} \in \mathrm{GL}(2 D+16 ; \mathbb{F})$ such that for each generator $\widehat{\rho}_{\alpha} \in \widehat{\mathbf{P}}$ there is a generator $\widehat{\rho}_{\alpha}^{\prime} \in \widehat{\mathbf{P}}^{\prime}$ with

$$
\widehat{\rho}_{\alpha}^{\prime}=\widehat{M}^{-1} \widehat{\rho}_{\alpha} \widehat{M} .
$$


Now, consider a Narain lattice spanned by a generalized vielbein $E$, such that $E$ is compatible with all generators $\widehat{\rho}_{\alpha}$ and insert eq. (6.3), i.e.

$$
\Theta_{\alpha} E=E \widehat{\rho}_{\alpha}=E \widehat{M} \widehat{\rho}_{\alpha}^{\prime} \widehat{M}^{-1} \text {. }
$$

Consequently, we find

$$
\Theta_{\alpha} E^{\prime}=E^{\prime} \widehat{\rho}_{\alpha}^{\prime} \quad \text { where } \quad E^{\prime}=E \widehat{M} .
$$

Hence, we can interpret eq. (6.5) as follows: if $\widehat{\mathbf{P}}$ is a symmetry of a Narain lattice with generalized vielbein $E$ and Narain metric $\widehat{\eta}$ then $\widehat{\mathbf{P}}^{\prime}$ is a symmetry of a Narain lattice with generalized vielbein $E^{\prime}=E \widehat{M}$ and Narain metric $\widehat{\eta}^{\prime}=\widehat{M}^{T} \widehat{\eta} \widehat{M}$. Furthermore, we notice that both point groups have the same geometrical action $\Theta_{\alpha}$ which corresponds to both $\widehat{\rho}_{\alpha}$ and $\widehat{\rho}_{\alpha}^{\prime}$. In other words, the corresponding point groups $\mathbf{P}$ and $\mathbf{P}^{\prime}$ in the coordinate basis are identical (up to a trivial basis change) for point groups from the same F-class. Consequently, the question of symmetric or asymmetric orbifolds, the number of unbroken supersymmetries in $d$ uncompactified dimensions and the number of invariant Narain moduli eq. (5.22) are also equal. This is independent of the choice for the field $\mathbb{F}$ to be $\mathbb{Q}$ or $\mathbb{Z}$.

Next, we have to distinguish between these two Narain classes: let us first consider the case $\mathbb{F}=\mathbb{Q}$. The Narain lattices spanned by $E$ and $E^{\prime}=E \widehat{M}$, are in general physically inequivalent, because if $\widehat{M} \in \mathrm{GL}(2 D+16$; Q $)$ then in general $\widehat{M} \notin \mathrm{GL}(2 D+16 ; \mathbb{Z})$. A representation of a $\mathbb{Q}$-class only gives one example of a compatible Narain lattice. To characterize all inequivalent lattices for a given $\mathbb{Q}$-class one needs to consider $\mathbb{Z}$-classes. That is, if $\mathbb{F}=\mathbb{Z}$ the generalized vielbeins $E$ and $E^{\prime}=E \widehat{M}$ span identical Narain lattices.

Finally, if $\widehat{M}$ additionally preserves the Narain metric $\widehat{\eta}$, i.e. if

$$
\widehat{M} \in \mathrm{O}_{\widehat{\eta}}(D, D+16 ; \mathbb{F}) \subset \mathrm{GL}(2 D+16 ; \mathbb{F}),
$$

which means that $\widehat{M}$ is a $T$-duality transformation, we can analyze the consequences of eq. (6.5) for the Narain moduli $G, B$ and $A$. In this case, we take the most general vielbein $E=U R \widehat{E}(e, B, A)$ from eq. (3.6) and use eq. (3.4) in order to transfer $\widehat{M}$ into $U_{\widehat{M}}$ for the generalized vielbein $E^{\prime}=E \widehat{M}$. Consequently, one can show that $E^{\prime}$ is given by

$$
E^{\prime}=(U R \widehat{E}(e, B, A)) \widehat{M}=U_{\mathrm{B}} U R \widehat{E}\left(e^{\prime}, B^{\prime}, A^{\prime}\right) \quad \text { where } \quad U_{\mathrm{B}}=U U_{\widehat{M}} U^{-1},
$$

and the $\widehat{M}$-transformed Narain moduli are given in eq. (3.14). Hence, if two Narain point groups $\widehat{\mathbf{P}}$ and $\widehat{\mathbf{P}}^{\prime}$ are $\mathbb{F}$-equivalent and defined with respect to the same Narain metric $\widehat{\eta}$ then the lattice $E=U R \widehat{E}(e, B, A)$ of $\widehat{\mathbf{P}}$ corresponds to the lattice $E^{\prime}$ of $\widehat{\mathbf{P}}^{\prime}$ as given in eq. (6.7). This change of lattices from $E$ to $E^{\prime}$ involves a transformation of moduli from $G, B$ and $A$ to $G^{\prime}, B^{\prime}$ and $A^{\prime}$ using the $T$-duality transformation $\widehat{M}$ and, in addition, a rotation in the coordinate basis with $U_{\mathrm{B}} \in \mathrm{O}(D ; \mathbb{R}) \times \mathrm{O}(D+16 ; \mathbb{R})$. Moreover, from eqs. (6.4) and (6.5) we obtain

$$
\begin{aligned}
\Theta_{\alpha}(U R \widehat{E}(e, B, A)) & =(U R \widehat{E}(e, B, A)) \widehat{\rho}_{\alpha}, \\
\left(U_{\mathrm{B}}^{-1} \Theta_{\alpha} U_{\mathrm{B}}\right)\left(U R \widehat{E}\left(e^{\prime}, B^{\prime}, A^{\prime}\right)\right) & =\left(U R \widehat{E}\left(e^{\prime}, B^{\prime}, A^{\prime}\right)\right) \widehat{\rho}_{\alpha}^{\prime} .
\end{aligned}
$$


That is, even though we have seen in eqs. (6.4) and (6.5) that the Narain point groups $\mathbf{P}$ and $\mathbf{P}^{\prime}$ are identical in the same $\mathbb{F}$-class, their generators $\Theta_{\alpha}$ and $\Theta_{\alpha}^{\prime}=U_{\mathrm{B}}^{-1} \Theta_{\alpha} U_{\mathrm{B}}$ can look different, for example, one is symmetric and the other looks asymmetric. This is the case if one chooses the corresponding Narain lattices as different points, specified by $(e, B, A)$ and $\left(e^{\prime}, B^{\prime}, A^{\prime}\right)$, in the same representation of the Narain moduli space, i.e. with the same $U$ in eq. (6.8). As an example for eq. (6.8), we will discus two $\mathbb{F}$-equivalent $\mathbb{Z}_{3}$

point groups $\widehat{\mathbf{P}}_{(1)}$ and $\widehat{\mathbf{P}}_{(2)}$ in section 8.4 , where the point group $\widehat{\mathbf{P}}_{(1)}$ is symmetric while $\widehat{\mathbf{P}}_{(2)}$ looks asymmetric due to a non-trivial transformation $U_{\mathrm{B}}$.

\subsection{Narain Poincaré-classes}

As final type of equivalence transformations, we want to generalize affine transformations $(F, \lambda)$ of Euclidean $D$-dimensional orbifolds (with linear mapping $F \in \mathrm{GL}(D ; \mathbb{R})$ and translation $\left.\lambda \in \mathbb{R}^{D}\right)$ to the Narain case. Importantly, the $(2 D+16)$-dimensional Narain lattice is equipped with a metric $\eta$ with signature $(D, D+16)$, which has to be preserved by any transformation. Hence, it is essential for the Narain case to restrict affine transformations in $2 D+16$ dimensions to Poincaré transformations $(F, \lambda)$ of the Narain lattice, where $F \in \mathrm{O}_{\eta}(D, D+16 ; \mathbb{R})$ and $\lambda \in \mathbb{R}^{2 D+16}$. Therefore, we need to introduce Poincaré-classes instead of affine classes in order to describe Narain orbifolds.

This might give the impression that Poincaré transformations of Narain orbifolds are more restrictive than affine transformations of ordinary Euclidean orbifolds. This is not the case since $\mathrm{O}_{\eta}(D, D+16 ; \mathbb{R})$ transformations contain $\mathrm{GL}(D ; \mathbb{R})$ transformations. This can be made explicit by the parametrization $E \widehat{M}_{e}(\Delta K) E^{-1} \in \mathrm{O}_{\eta}(D, D+16 ; \mathbb{R})$, where $\widehat{M}_{e}(\Delta K)$ is is given in table 1 with $\Delta K \in \mathrm{GL}(D ; \mathbb{R})$. Consequently, Poincaré-classes generalize the notion of affine classes to Narain orbifolds.

In light of this, we define the following equivalence relation: consider two Narain orbifolds, i.e. two space groups $\mathbf{S}_{(1)}$ and $\mathbf{S}_{(2)}$ with point groups in the same $\mathbb{Z}$-class. Two such Narain space groups are defined to be equivalent if there exists a Poincaré transformation $(F, \lambda)$ with $F \in \mathrm{O}_{\eta}(D, D+16 ; \mathbb{R})$ and $\lambda \in \mathbb{R}^{2 D+16}$ such that

$$
\mathbf{S}_{(2)}=(F, \lambda)^{-1} \mathbf{S}_{(1)}(F, \lambda) .
$$

More explicitly, in terms of the generators $\left(\Theta_{(\kappa) \alpha}, V_{(\kappa) \alpha}\right)$ and $\left(\mathbb{1}, L_{(\kappa)}\right)$ of the space groups $\mathbf{S}_{(\kappa)}$ for $\kappa=1,2$ this reads

$$
L_{(2)}=F^{-1} L_{(1)}, \quad \Theta_{(2) \alpha}=F^{-1} \Theta_{(1) \alpha} F, \quad V_{(2) \alpha}=F^{-1}\left(V_{(1) \alpha}-\left(\mathbb{1}-\Theta_{(1) \alpha}\right) \lambda\right),
$$

see eq. (4.6). Notice that Narain $\mathbb{Q}$ - and $\mathbb{Z}$-classes involve transformations in the lattice basis, while Narain Poincaré classes involve transformations in the coordinate basis. Since Narain Poincaré transformations act on all defining quantities of the space group, see eq. (6.10), their interpretation is more involved.

\subsection{Interpretation of Narain Poincaré-classes}

First of all, we show that two generalized space groups from the same affine class correspond to the same Narain orbifold but possibly at different points in the moduli space. To 
see this, let us denote the generalized vielbeins that specify the Narain lattices from the respective generalized space groups $\mathbf{S}_{(\kappa)}$ by $E_{(\kappa)}=U_{(\kappa)} R \widehat{E}\left(e_{(\kappa)}, B_{(\kappa)}, A_{(\kappa)}\right)$ for $\kappa=1,2$, where $U_{(\kappa)} \in \mathrm{O}(D ; \mathbb{R}) \times \mathrm{O}(D+16 ; \mathbb{R})$ and $\widehat{E}\left(e_{(\kappa)}, B_{(\kappa)}, A_{(\kappa)}\right)$ is given in eq. (3.1). Since $L_{(\kappa)}=E_{(\kappa)} N_{(\kappa)}$ are related by the transformation (6.10), a Poincaré transformation $(F, \lambda)$ of the corresponding generalized vielbeins $E_{(1)}$ and $E_{(2)}$ is given by

$$
U_{(2)} R \widehat{E}\left(e_{(2)}, B_{(2)}, A_{(2)}\right)=E_{(2)}=F^{-1} E_{(1)}=F^{-1} U_{(1)} R \widehat{E}\left(e_{(1)}, B_{(1)}, A_{(1)}\right),
$$

where we assume without loss of generality that we do not perform a discrete $T$-duality transformation (i.e. $\left.N_{(2)}=N_{(1)}\right)$. This can be rewritten as

$$
\widehat{U}_{(2)} \widehat{E}\left(e_{(2)}, B_{(2)}, A_{(2)}\right)=\widehat{E}\left(e_{(1)}, B_{(1)}, A_{(1)}\right) \widehat{M}_{F},
$$

where

$$
\widehat{U}_{(2)}=R^{-1} U_{(2)} R \text { and } \widehat{M}_{F}=E_{(1)}^{-1} U_{(1)} F^{-1} E_{(1)} \in \mathrm{O}_{\widehat{\eta}}(D, D+16, \mathbb{R}) .
$$

Since $\widehat{M}_{F}$ parametrizes a general $T$-duality transformation, we can make use of eq. (3.4) to determine the transformation of the moduli by setting $\widehat{M}=\widehat{M}_{F}$, i.e.

$$
\widehat{U}_{\widehat{M}_{F}} \widehat{E}\left(e_{(1)}^{\prime}, B_{(1)}^{\prime}, A_{(1)}^{\prime}\right)=\widehat{E}\left(e_{(1)}, B_{(1)}, A_{(1)}\right) \widehat{M}_{F} .
$$

Since the generalized vielbein is uniquely defined up to $\mathrm{O}(D ; \mathbb{R}) \times \mathrm{O}(D+16 ; \mathbb{R})$ transformations, we conclude that

$$
e_{(2)}=e_{(1)}^{\prime}, \quad B_{(2)}=B_{(1)}^{\prime}, \quad A_{(2)}=A_{(1)}^{\prime},
$$

where the prime denotes the resulting moduli under the $T$-duality transformation $\widehat{M}_{F}$. This tells us that two generalized space groups from the same Poincaré-class can correspond to the same Narain orbifold but at different points in the moduli space. In fact only if $F \in \mathrm{O}(D ; \mathbb{R}) \times \mathrm{O}(D+16 ; \mathbb{R}) \backslash \mathrm{O}_{\eta}(D, D+16 ; \mathbb{R})$ we get a proper moduli transformation. Indeed, if $F \in \mathrm{O}(D ; \mathbb{R}) \times \mathrm{O}(D+16 ; \mathbb{R})$ we find that $U_{(2)}=F^{-1} U_{(1)}$ as well as $e_{(2)}=e_{(1)}$, $B_{(2)}=B_{(1)}$ and $A_{(2)}=A_{(1)}$. In this case, also the left- and right-moving mass formulae of the heterotic string stay the same.

So far we only gave an interpretation of the first equivalence relation in eqs. (6.10). The second relation tells us that the orbifold twists can take various guises by conjugation with $F \in \mathrm{O}_{\eta}(D, D+16 ; \mathbb{R})$. The third equivalence relation in eqs. (6.10) can be interpreted by resorting to the decomposition mentioned in section 4.5 .

\section{$7 \quad$ Symmetric orbifolds as Narain orbifolds}

The main objective of our study in this paper is to set up a framework to investigate asymmetric orbifolds. Nevertheless, it is very instructive to apply the Narain formalism also to symmetric orbifolds $[5,6]$ : it provides us with a unified view on both, geometric moduli and Wilson lines [8]. Moreover, this case can be used to illustrate the power of the $T$-duality group approach in the investigation of moduli stabilization. For concreteness and simplicity, we only consider symmetric $\mathbb{Z}_{K}$ orbifolds in this section. Extending the discussion is straightforward, yet beyond the scope of the present paper. 


\subsection{Symmetric $\mathbb{Z}_{K}$ orbifolds}

The Narain point group of a symmetric $\mathbb{Z}_{K}$ orbifold is generated by a single twist $\Theta$ of order $K$ and the associated generator of the generalized space group is given by $(\Theta, V)$. For the orbifold to be symmetric, we choose the twist $\Theta$ to be of the form given in eq. (4.5). Thus, we obtain for $\Theta^{k}, k=1, \ldots, K$,

$$
\widehat{\Theta}^{k}=R^{-1} \Theta^{k} R=\left(\begin{array}{ccc}
\theta^{k} & 0 & 0 \\
0 & \theta^{k} & 0 \\
0 & 0 & \mathbb{1}_{16}
\end{array}\right)=\widehat{M}_{e}\left(\theta^{k}\right),
$$

see table 1 and using $\theta^{T} \theta=\mathbb{1}_{D}$. Using the definition (5.1) of the integral matrix $\widehat{\rho}$ we can subsequently obtain an expression for $\widehat{\rho}^{k}$, which can be further evaluated with the help of the multiplication table 2 for $T$-duality group elements. This yields

$$
\widehat{\rho}^{k}=\widehat{E}(e, B, A)^{-1} \widehat{\Theta}^{k} \widehat{E}(e, B, A)=\widehat{M}_{e}\left(\hat{\theta}^{k}\right) \widehat{M}_{B}\left(\Delta B_{k}\right) \widehat{M}_{A}\left(\Delta A_{k}\right),
$$

where we defined

$$
\begin{aligned}
\hat{\theta} & =e^{-1} \theta e \\
\Delta B_{k} & =B-\hat{\theta}^{k T} B \hat{\theta}^{k}+\frac{1}{2}\left(\hat{\theta}^{k T} A^{T} A-A^{T} A \hat{\theta}^{k}\right) \quad \text { with } \quad \Delta B_{k}^{T}=-\Delta B_{k} \\
\Delta A_{k} & =A\left(\mathbb{1}_{D}-\hat{\theta}^{k}\right) .
\end{aligned}
$$

Since $\hat{\rho}$ is an integral matrix, $\hat{\theta}, \Delta B_{k}$ and $\Delta A_{k}$ all have to be constant, i.e. moduliindependent, matrices. As a cross-check, let us confirm that for $k=K$ we obtain $\widehat{\rho}^{K}=\mathbb{1}$ : indeed, in this case we get $\hat{\theta}^{K}=\mathbb{1}_{D}, \Delta A_{K}=0$ and $\Delta B_{K}=0$ and consequently, $\widehat{\rho}^{K}=\widehat{M}_{e}\left(\mathbb{1}_{D}\right)=\mathbb{1}$, as required. Furthermore, we find from eq. (7.2) that $\widehat{\rho}$ is an element of the discrete geometric subgroup $G_{\text {geom }}(\mathbb{Z}) \subset \mathrm{O}_{\hat{\eta}}(D, D+16 ; \mathbb{Z})$, see eq. (3.15) with $\Delta W=\mathbb{1}_{16}$.

The twist $\Theta$ is in general accompanied by a shift $V^{T}=\left(V_{\mathrm{r}}^{T}, V_{\mathrm{l}}^{T}, V_{\mathrm{L}}^{T}\right)$, see eq. (4.6). As we have seen in section 4.5, the shift is quantized, i.e. $K V_{\|}^{\ominus}=E N_{V} \in \mathbb{\Gamma}$. It is instructive to analyze this in more detail for the case that $\theta$ rotates in all $D$ compact dimensions. Then, the projection operator eq. (4.15) reads

$$
\mathcal{P}_{\|}^{\Theta}=\left(\begin{array}{lll}
0 & 0 & 0 \\
0 & 0 & 0 \\
0 & 0 & \mathbb{1}_{16}
\end{array}\right),
$$

and we obtain the condition

$$
K V_{\|}^{\ominus}=\left(\begin{array}{c}
0 \\
0 \\
K V_{\mathrm{L}}
\end{array}\right) \stackrel{!}{=} E N_{V} \in \mathbb{\Gamma} \quad \text { with } \quad N_{V}=\left(\begin{array}{c}
m_{V} \\
n_{V} \\
q_{V}
\end{array}\right) \in \mathbb{Z}^{2 D+16} .
$$

This is solved by

$$
K V_{\mathrm{L}}=\alpha_{\mathrm{g}} q_{V} \in \Lambda_{\mathrm{E}_{8} \times \mathrm{E}_{8}}, \quad K A^{T} V_{\mathrm{L}}=n_{V} \in \mathbb{Z}^{6} \quad \text { and } \quad m_{V}=0,
$$


where $\Lambda_{\mathrm{E}_{8} \times \mathrm{E}_{8}}$ denotes the root lattice of $\mathrm{E}_{8} \times \mathrm{E}_{8}$ and we used eq. (2.44). Hence, $V_{\mathrm{L}}$ is the gauge shift vector of order $K$ known to the symmetric orbifold literature, e.g. [13, 52]. Furthermore, we can set $V_{\mathrm{r}}=V_{\mathrm{l}}=0$ by shifting the origin using the transformation (4.23).

\subsection{Moduli stabilization in symmetric $\mathbb{Z}_{K}$ orbifolds}

The fact that even for symmetric $\mathbb{Z}_{K}$ orbifolds a certain number of moduli, $G, B$ and $A$, become constrained, can be inferred in two ways: first of all, the conditions (7.3) can be obtained from eqs. (7.2), as shown above by using the fact that for symmetric orbifolds the twist $\widehat{\rho}$ is an element of the geometric subgroup $G_{\text {geom }}(\mathbb{Z}) \subset \mathrm{O}_{\hat{\eta}}(D, D+16 ; \mathbb{Z})$. A second derivation of eq. (7.3) follows from the general discussion in section 5.2, which is valid for both, symmetric and asymmetric orbifolds: to see this, we use

$$
\widehat{\rho}=\widehat{M}_{e}(\hat{\theta}) \widehat{M}_{B}\left(\Delta B_{1}\right) \widehat{M}_{A}\left(\Delta A_{1}\right)=\widehat{E}\left(\hat{\theta}, \Delta B_{1}, \Delta A_{1}\right),
$$

see eq. (7.2) and eq. (3.1). Then, we set $\widehat{M}=\widehat{\rho}$ in eq. (3.8) and obtain

$$
\begin{aligned}
& \widehat{M}_{1}=\hat{\theta}^{-T}\left(-\Delta B_{1}+\frac{1}{2} \Delta A_{1}^{T} \Delta A_{1}\right)+\left(G+C^{T}\right) \hat{\theta}+A^{T} \Delta A_{1}, \\
& \widehat{M}_{2}=-\hat{\theta}^{-T}, \quad \widehat{M}_{3}=\left(\Delta A_{1} \hat{\theta}^{-1}+A\right)^{T} \alpha_{\mathrm{g}},
\end{aligned}
$$

and in addition we have $\rho_{\mathrm{r}}=e^{-1} \theta_{\mathrm{r}} e=\hat{\theta}$. Consequently, the Narain moduli are constrained by eqs. (5.16), which are equivalent to eqs. (7.3). Thus, we found two equivalent ways to derive the conditions (7.3) for Narain moduli stabilization in the case of symmetric orbifolds.

Let us now discuss the consequences of eqs. (7.3) for Narain moduli stabilization. Since $\widehat{\rho}$ in eq. (7.7) has to be an integer matrix, i.e. $\widehat{\rho} \in \mathrm{O}_{\hat{\eta}}(D, D+16 ; \mathbb{Z})$, we have to demand that

$$
\hat{\theta} \in \operatorname{GL}(D ; \mathbb{Z}), \quad \alpha_{\mathrm{g}}^{-1} \Delta A_{k} \in M_{16 \times D}(\mathbb{Z}), \quad-\frac{1}{2} \Delta A_{k}^{T} \Delta A_{k}+\Delta B_{k} \in M_{D \times D}(\mathbb{Z}),
$$

as can be inferred from eqs. (2.39) and (3.24).

We start with fixing moduli in the metric $G$. From eq. (7.3a) and $\Theta^{T} \Theta=\mathbb{1}$ we obtain the condition

$$
\hat{\theta}^{T} G \hat{\theta} \stackrel{!}{=} G \quad \Leftrightarrow \quad \hat{\theta} \in O_{G}(D ; \mathbb{Z}),
$$

which fixes some of the moduli, as is well-known. The general solution to eq. (7.10) for a given $\hat{\theta}$ can be parametrized as

$$
G=\frac{1}{K} \sum_{k=0}^{K-1} \hat{\theta}^{k T} G_{0} \hat{\theta}^{k}
$$

where $G_{0}$ is some symmetric positive definite matrix, for example $G_{0}=\mathbb{1}_{D}$. Now, it is easy to demonstrate that some metric moduli remain unconstrained for symmetric orbifolds: at least we can scale $G_{0}$ with an arbitrary positive factor, while eq. (7.10) stays fulfilled.

Next, we consider the Wilson lines. If $\theta$ rotates in all $D$ compact dimensions $\left(\mathbb{1}_{D}-\hat{\theta}\right)$ is invertible, i.e.

$$
\left(\mathbb{1}_{D}-\hat{\theta}\right)^{-1}=-\frac{1}{K} \sum_{n=1}^{K-1} n \hat{\theta}^{n}
$$


and the Wilson lines are uniquely determined from $\Delta A_{k}$ in eq. (7.3c), e.g. from $k=1$

$$
A \stackrel{!}{=}-\frac{1}{K} \sum_{n=1}^{K-1} n \Delta A_{1} \hat{\theta}^{n} .
$$

Consequently, the Wilson lines $A$ are completely frozen as they have to be discrete, i.e. quantized in units of $1 / K$ in the directions where $\theta$ acts non-trivially. As a further consequence of eq. (7.3c) we see that two Wilson lines (i.e. two columns of $A$ ) have to be identical up to some trivial $\Delta A_{k}$ if the corresponding columns in the geometrical vielbein $e$ are mapped to each other by $\hat{\theta}^{k}$.

Finally, the $B$-field is constrained by the condition (7.3b)

$$
B-\hat{\theta}^{T} B \hat{\theta} \stackrel{!}{=} \Delta B=\Delta B_{1}-\frac{1}{2}\left(\hat{\theta}^{T} A^{T} A-A^{T} A \hat{\theta}\right),
$$

combined with eq. (7.9). In analogy to eq. (7.11) the general solution of this equation can written as

$$
B=\frac{1}{K} \sum_{k=0}^{K-1} \hat{\theta}^{k T} B_{0} \hat{\theta}^{k}+B_{P},
$$

where $B_{0}$ is an arbitrary anti-symmetric matrix (for example, $B_{0}=0$ ) and $B_{P}$ is a particular solution to eq. (7.14). For example, in $D=2$ the anti-symmetric $2 \times 2$ matrix $B$ contains a single modulus. It is subject to eq. (7.14), i.e.

$$
B-\hat{\theta}^{T} B \hat{\theta}=(1-\operatorname{det}(\hat{\theta})) B \stackrel{!}{=} \Delta B,
$$

where $\operatorname{det}(\hat{\theta})= \pm 1$. Thus, for $\operatorname{det}(\hat{\theta})=1$ we obtain $\Delta B \stackrel{!}{=} 0$ and the single $B$-field modulus in $B$ is unconstrained and $B_{P}=0$. On the other hand, $B$ is stabilized at $B_{P}=\frac{1}{2} \Delta B$ if $\operatorname{det}(\hat{\theta})=-1$.

Number of moduli in symmetric $\mathbb{Z}_{\boldsymbol{K}}$ orbifolds. We can compute the number of (real) unstabilized moduli for symmetric $\mathbb{Z}_{K}$ orbifolds for general $K$ using the results of section 5.4. To do so, we assume for simplicity $D=6$ and $K \neq 2$. Furthermore, we choose a $\mathbb{Z}_{K}$ twist vector $\phi_{\mathrm{R}}=\left(0, \phi_{\mathrm{R}}^{1}, \phi_{\mathrm{R}}^{2},-\phi_{\mathrm{R}}^{1}-\phi_{\mathrm{R}}^{2}\right)$ such that $\mathcal{N}=1$ supersymmetry survives in four dimensions, see section 4.6. Hence, $K=3,4,6,7,8$ or 12. Then, eq. (5.22) yields

$$
\begin{aligned}
\operatorname{dim}\left(\mathcal{M}_{\mathbb{Z}_{K}}\right)= & 6+2\left(\delta_{\phi_{\mathrm{R}}^{1}, \frac{1}{2}}+\delta_{\phi_{\mathrm{R}}^{2}, \frac{1}{2}}+\delta_{\phi_{\mathrm{R}}^{1}+\phi_{\mathrm{R}}^{2}, \frac{1}{2}}\right) \\
& +4\left(\delta_{\phi_{\mathrm{R}}^{1}, \phi_{\mathrm{R}}^{2}}+\delta_{\phi_{\mathrm{R}}^{1},-\phi_{\mathrm{R}}^{2}}+\delta_{\phi_{\mathrm{R}}^{1},-2 \phi_{\mathrm{R}}^{2}}+\delta_{2 \phi_{\mathrm{R}}^{1},-\phi_{\mathrm{R}}^{2}}\right),
\end{aligned}
$$

where $\delta_{a, b}=1$ if $a \equiv b$ and $\delta_{a, b}=0$ otherwise. For example, for $\mathbb{Z}_{3}$ we take $\phi_{\mathrm{R}}^{1}=\phi_{\mathrm{R}}^{2}=\frac{1}{3}$ and obtain $\operatorname{dim}\left(\mathcal{M}_{\mathbb{Z}_{3}}\right)=6+2 \times 0+4 \times(1+0+1+1)=18$. As is well-known, these 18 (real) moduli correspond to 9 complex structure moduli, see e.g. [90].

\section{Two-dimensional Abelian Narain orbifolds}

In this section, we study examples of generalized space groups of Narain orbifolds with Abelian Narain point groups $\mathbb{Z}_{K}$ in two dimensions. Many of them correspond to previously 
unknown two-dimensional Narain orbifolds. We collect them in a comprehensive table. Furthermore, to illustrate various aspects of the theory developed in previous sections, we describe some of these two-dimensional $\mathbb{Z}_{K}$ Narain orbifolds in more detail. For example, by an explicit construction we show that it is possible to have $\mathbb{Z}_{12}$ two-dimensional Narain orbifolds, while it is well-known that for Euclidean orbifolds in $D=2$ the largest order of a twist is $K=6$. Moreover, the $\mathbb{Q}$ - and $\mathbb{Z}$-classes are used to distinguish seemingly asymmetric from truly asymmetric orbifolds.

\section{1 (D,D)-Narain orbifold formalism}

To prepare the discussion of various illustrative examples of two-dimensional Narain orbifolds, we briefly restrict the Narain orbifold formalism to the case where $\eta$ has signature $(D, D)$ :

$$
\widehat{\eta}=R^{T} \eta R=\left(\begin{array}{cc}
0 & \mathbb{1}_{D} \\
\mathbb{1}_{D} & 0
\end{array}\right) \quad \text { with } \quad R=\frac{1}{\sqrt{2}}\left(\begin{array}{cc}
\mathbb{1}_{D} & -\mathbb{1}_{D} \\
\mathbb{1}_{D} & \mathbb{1}_{D}
\end{array}\right) .
$$

The generalized vielbein $\widehat{E}$ is an element from $\mathrm{O}_{\widehat{\eta}}(D, D ; \mathbb{R})$,

$$
\widehat{E}(e, B)=\left(\begin{array}{cc}
e & 0 \\
e^{-T} B & e^{-T}
\end{array}\right) .
$$

Analogously to the discussion in section 3.3, for each element

$$
\widehat{M}=\left(\begin{array}{ll}
\widehat{M}_{11} & \widehat{M}_{12} \\
\widehat{M}_{21} & \widehat{M}_{22}
\end{array}\right) \in \mathrm{O}_{\widehat{\eta}}(D, D ; \mathbb{R}),
$$

there exist a choice for a matrix $U_{\widehat{M}} \in O(D ; \mathbb{R}) \times O(D ; \mathbb{R})$ and transformed moduli $e^{\prime}$ and $B^{\prime}$, such that

$$
\widehat{U}_{\widehat{M}} \widehat{E}\left(e^{\prime}, B^{\prime}\right)=\widehat{E}(e, B) \widehat{M}, \quad U_{\widehat{M}}=R \widehat{U}_{\widehat{M}} R^{-1}=\left(\begin{array}{cc}
u_{\mathrm{r}} & 0 \\
0 & u_{\mathrm{l}}
\end{array}\right) .
$$

In detail, defining

$$
\widehat{M}_{1}=-\widehat{M}_{21}+(G-B) \widehat{M}_{11} \quad \text { and } \quad \widehat{M}_{2}=-\widehat{M}_{22}+(G-B) \widehat{M}_{12},
$$

in accordance with eq. (3.8), we obtain

$$
u_{\mathrm{l}}=\left(\mathbb{1}_{D}-2 e \widehat{M}_{12} \widehat{M}_{2}^{-1} e^{T}\right) u_{\mathrm{r}} \in O(D ; \mathbb{R})
$$

for arbitrary $u_{\mathrm{r}} \in O(D ; \mathbb{R})$. This shows that $\widehat{M}_{12} \neq 0$ is a necessary condition for $u_{\mathrm{r}} \neq u_{\mathrm{l}}$. Furthermore, the Narain moduli transform as

$$
e^{\prime}=-u_{\mathrm{r}}^{-1} e \widehat{M}_{2}^{-T}, \quad G^{\prime}=\widehat{M}_{2}^{-1} G \widehat{M}_{2}^{-T}, \quad B^{\prime}=\frac{1}{2}\left(\widehat{M}_{2}^{-1} \widehat{M}_{1}-\widehat{M}_{1}^{T} \widehat{M}_{2}^{-T}\right) .
$$

By restricting $\widehat{M}$ to lie either inside $\mathrm{O}_{\widehat{\eta}}(D, D ; \mathbb{Q})$ or $\mathrm{O}_{\widehat{\eta}}(D, D ; \mathbb{Z})$, we obtain the transformations that map different representations within the same $\mathbb{Q}$ - or $\mathbb{Z}$-class to each other. 
Next, we discuss Narain orbifolds with Abelian $\mathbb{Z}_{K}$ point groups $\widehat{\mathbf{P}} \subset \mathrm{O}_{\widehat{\eta}}(D, D ; \mathbb{Z})$. We use eq. (8.5) and set $\widehat{M}=\widehat{\rho}$, where $\widehat{\rho}$ is the generator of $\widehat{\mathbf{P}}$. Then, we find invariant moduli $G^{\prime}=G$ and $B^{\prime}=B$ from the latter two transformations in eqs. (8.7). Moreover, we obtain the right-moving twist $\theta_{\mathrm{r}}=u_{\mathrm{r}}$ from the first relation in eq. (8.7) by choosing a vielbein $e^{\prime}=e$, which is in agreement with $G^{\prime}=G$. By identifying the full twist $\widehat{\Theta}=\widehat{U}_{\widehat{\rho}}$ from eq. (8.6) the Narain orbifold condition follows from eq. (8.4), i.e.

$$
\widehat{\Theta} \widehat{E}(e, B)=\widehat{E}(e, B) \widehat{\rho} .
$$

Then, in analogy to section 5.2 we know that the $\mathbb{Z}_{K}$ Narain orbifold exists.

If the matrix-block $\widehat{\rho}_{12}$ is zero the orbifold is symmetric (i.e. $\theta_{\mathrm{r}}=\theta_{\mathrm{l}}$ ) and a necessary (but not sufficient) condition for the orbifold to be asymmetric is $\widehat{\rho}_{12} \neq 0$, as can be seen from eq. (8.6).

\subsection{Q- and $\mathbb{Z}$-classes of two-dimensional $\mathbb{Z}_{K}$ Narain orbifolds}

Following the discussion of the last section we focus on two-dimensional Narain orbifolds with point groups $\widehat{\mathbf{P}} \subset \mathrm{O}_{\widehat{\eta}}(2,2 ; \mathbb{Z})$, generated by a single twist $\widehat{\rho}$ of order $K$.

To initiate this investigation, we give a brief discussion on the possible orders following section 4.3: for Narain orbifolds with $D=2$ we have to set $D_{\Gamma}=2 D=4$. Then, eq. (4.14) yields the following list of possible orders

$$
K \in\{1,2,3,4,5,6,8,10,12\} .
$$

In contrast, for two-dimensional symmetric orbifolds we have $D_{\Gamma}=D=2$ which yields only $K \in\{1,2,3,4,6\}$. Indeed, as we discuss in the following, we found examples for $K=12$. They are genuine asymmetric because twists of order 12 are not possible for $D_{\Gamma}=2$. On the other hand, we did not find any examples for $K=5,8$ and 10 in the scan of two-dimensional Narain orbifold we performed for this paper.

In table 3 we list a number of Abelian $\mathbb{Z}_{K}$ Narain orbifolds of order $K$, which we constructed explicitly in our scan. For each Narain point group $\widehat{\mathbf{P}} \subset \mathrm{O}_{\widehat{\eta}}(2,2 ; \mathbb{Z})$ this table displays the following data in the various columns:

1. column labels the inequivalent orbifolds and characterizes the orbifold as symmetric or asymmetric;

2. column gives a representation of the generating twist $\widehat{\rho}$ of order $K$ in the lattice basis;

3. column displays the corresponding right-twist $\theta_{\mathrm{r}}$;

4. column displays the corresponding left-twist $\theta_{1}$;

5. column indicates the relation between these twists;

6. column gives a choice of the geometrical vielbein $e$;

7. column gives to resulting metric as $G=e^{T} e$; 
8. column gives the anti-symmetric $B$-field.

A couple of further comments about the conventions of this table are in order: our labelling conventions for inequivalent Narain orbifolds are as follows. The inequivalent $\mathbb{Q}$-classes of a given order $K$ are enumerated by a Roman number $\mathrm{R}=\mathrm{I}, \mathrm{II}, \ldots$ as $\mathbb{Z}_{K}-\mathrm{R}$. Furthermore, when we give inequivalent $\mathbb{Z}$-classes within a given $\mathbb{Q}$-class, we enumerate them with $n=1,2,3$ as $\mathbb{Z}_{K^{-}}$R-n. In fact, only the $\mathbb{Q}$-class $\mathbb{Z}_{2}$-II is subdivided into three inequivalent $\mathbb{Z}$-classes. Furthermore, the given right- and left-twists depend on our choice for the geometrical vielbein $e$ and on the Narain moduli $G$ and $B$.

To describe all these two dimensional Narain orbifolds in detail would lead to a lengthy discussion. Therefore, we focus in the following subsections on a number of striking features of some of these orbifolds instead. Before, doing so we make a couple of observations: first of all, we see that the number of asymmetric orbifolds greatly outweighs the number of symmetric orbifolds. This might imply that there exist many more asymmetric Narain orbifolds than symmetric ones. Most of the asymmetric orbifolds constructed in the past have twists that are trivial for either the left- or the right-moving sectors, like the $\mathbb{Z}_{3}$-II and $\mathbb{Z}_{3}$-III orbifolds. In our scan we also encountered such examples, but again it seems that the majority of asymmetric orbifolds are not of this type: most of them have non-trivial left- and right-moving twists simultaneously. In fact, there are even cases where the orders of the left- and right-moving twists are co-prime: the $\mathbb{Z}_{6}$-IV and $\mathbb{Z}_{6}$-VII Narain orbifolds. Since their orders are coprime, all their characters are orthogonal. Using the results of section 5.4 this immediately implies that all moduli are stabilized for these orbifolds.

\subsection{Two equivalent asymmetric $\mathbb{Z}_{12}$ Narain orbifolds}

With our first two examples we want to illustrate that we are able to construct genuine asymmetric orbifolds using the formalism for Narain orbifolds exposed in this paper. Concretely, we define two $\mathbb{Z}_{12}$ Narain point groups $\widehat{\mathbf{P}}_{(1)}$ and $\widehat{\mathbf{P}}_{(1)}$ in $D=2$, each being generated by an element $\widehat{\rho}_{(1)}, \widehat{\rho}_{(2)} \in \mathrm{O}_{\widehat{\eta}}(2,2 ; \mathbb{Z})$ of order 12 . In each case, we determine the corresponding Narain lattice and the twist $\Theta$ which is given by its action on rightand left-movers, $\theta_{\mathrm{r}}$ and $\theta_{\mathrm{l}}$, respectively. As there is no symmetric $\mathbb{Z}_{12}$ orbifold in $D=2$ (i.e. there is no two-dimensional lattice with rotational symmetry of order 12), these orbifolds must be genuine asymmetric. ${ }^{3}$ Moreover, to emphasize that the use of $\mathbb{Z}$-classes is extremely powerful to investigate whether two orbifolds are distinct, we show that these two $\mathbb{Z}_{12}$ point groups are in fact equivalent by giving an explicit $\mathrm{O}_{\widehat{\eta}}(D, D ; \mathbb{Z})$ matrix that relates the two twists in the lattice basis.

The first asymmetric $\mathbb{Z}_{12}$ orbifold example has a non-vanish $B$-field $B_{(1)} \neq 0$ : we choose

$$
\widehat{\rho}_{(1)}=\left(\begin{array}{cc}
0 & \mathbb{1}_{2} \\
\mathbb{1}_{2} & \epsilon
\end{array}\right) \in \mathrm{O}_{\widehat{\eta}}(2,2 ; \mathbb{Z}) \quad \text { where } \quad \epsilon=\left(\begin{array}{cc}
0 & 1 \\
-1 & 0
\end{array}\right)
$$

and obtain

$$
\widehat{M}_{(1) 1}=-\mathbb{1}_{2}, \quad \widehat{M}_{(1) 2}=G_{(1)}-B_{(1)}-\epsilon,
$$

\footnotetext{
${ }^{3}$ Such asymmetric $\mathbb{Z}_{12}$ orbifolds were studied in the past [51, 91].
} 


\begin{tabular}{|c|c|c|c|c|c|c|c|}
\hline label & twist $\widehat{\rho}$ & twist $\theta_{\mathrm{r}}$ & twist $\theta_{1}$ & relation & vielbein $e$ & metric $G$ & $B$-field \\
\hline $\begin{array}{l}\mathbb{Z}_{2} \text {-I } \\
\text { sym. }\end{array}$ & $\left(\begin{array}{cccc}-1 & 0 & 0 & 0 \\
0 & -1 & 0 & 0 \\
0 & 0 & -1 & 0 \\
0 & 0 & 0 & -1\end{array}\right)$ & $-\mathbb{1}_{2}$ & $-\mathbb{1}_{2}$ & $\theta_{\mathrm{r}}=\theta_{\mathrm{l}}$ & $\left(\begin{array}{cc}R_{1} & R_{2} \cos \alpha \\
0 & R_{2} \sin \alpha\end{array}\right)$ & $\begin{array}{c}\left(\begin{array}{cc}R_{1}^{2} & w \\
w & R_{2}^{2}\end{array}\right) \\
w=R_{1} R_{2} \cos \alpha\end{array}$ & $\left(\begin{array}{cc}0 & b \\
-b & 0\end{array}\right)$ \\
\hline $\begin{array}{c}\mathbb{Z}_{2} \text {-II-1 } \\
\text { sym. }\end{array}$ & $\left(\begin{array}{cccc}1 & 0 & 0 & 0 \\
0 & -1 & 0 & 0 \\
0 & 0 & 1 & 0 \\
0 & 0 & 0 & -1\end{array}\right)$ & $\left(\begin{array}{cc}1 & 0 \\
0 & -1\end{array}\right)$ & $\left(\begin{array}{cc}1 & 0 \\
0 & -1\end{array}\right)$ & $\theta_{\mathrm{r}}=\theta_{\mathrm{l}}$ & $\left(\begin{array}{cc}R_{1} & 0 \\
0 & R_{2}\end{array}\right)$ & $\left(\begin{array}{cc}R_{1}^{2} & 0 \\
0 & R_{2}^{2}\end{array}\right)$ & $\left(\begin{array}{ll}0 & 0 \\
0 & 0\end{array}\right)$ \\
\hline $\begin{array}{c}\mathbb{Z}_{2} \text {-II-2 } \\
\text { sym. }\end{array}$ & $\left(\begin{array}{llll}0 & 1 & 0 & 0 \\
1 & 0 & 0 & 0 \\
0 & 0 & 0 & 1 \\
0 & 0 & 1 & 0\end{array}\right)$ & $\left(\begin{array}{cc}1 & 0 \\
0 & -1\end{array}\right)$ & $\left(\begin{array}{cc}1 & 0 \\
0 & -1\end{array}\right)$ & $\theta_{\mathrm{r}}=\theta_{\mathrm{l}}$ & $\left(\begin{array}{cc}R_{1} & R_{1} \\
-R_{2} & R_{2}\end{array}\right)$ & $\left(\begin{array}{ll}R_{1}^{2}+R_{2}^{2} & R_{1}^{2}-R_{2}^{2} \\
R_{1}^{2}-R_{2}^{2} & R_{1}^{2}+R_{2}^{2}\end{array}\right)$ & $\left(\begin{array}{ll}0 & 0 \\
0 & 0\end{array}\right)$ \\
\hline $\begin{array}{c}\mathbb{Z}_{2} \text {-II-3 } \\
\text { sym. }\end{array}$ & $\left(\begin{array}{cccc}0 & 1 & 0 & 0 \\
1 & 0 & 0 & 0 \\
-1 & 0 & 0 & 1 \\
0 & 1 & 1 & 0\end{array}\right)$ & $\left(\begin{array}{cc}1 & 0 \\
0 & -1\end{array}\right)$ & $\left(\begin{array}{cc}1 & 0 \\
0 & -1\end{array}\right)$ & $\theta_{\mathrm{r}}=\theta_{\mathrm{l}}$ & $\left(\begin{array}{cc}R_{1} & R_{1} \\
-R_{2} & R_{2}\end{array}\right)$ & $\left(\begin{array}{ll}R_{1}^{2}+R_{2}^{2} & R_{1}^{2}-R_{2}^{2} \\
R_{1}^{2}-R_{2}^{2} & R_{1}^{2}+R_{2}^{2}\end{array}\right)$ & $\left(\begin{array}{cc}0 & \frac{1}{2} \\
-\frac{1}{2} & 0\end{array}\right)$ \\
\hline $\begin{array}{l}\mathbb{Z}_{3} \text {-I } \\
\text { sym. }\end{array}$ & $\left(\begin{array}{cccc}0 & -1 & 0 & 0 \\
1 & -1 & 0 & 0 \\
0 & 0 & -1 & -1 \\
0 & 0 & 1 & 0\end{array}\right)$ & $\left(\begin{array}{ll}-\frac{1}{2} & -\frac{\sqrt{2}}{2} \\
\frac{\sqrt{3}}{2} & -\frac{1}{2}\end{array}\right)$ & $\left(\begin{array}{cc}-\frac{1}{2} & -\frac{\sqrt{3}}{2} \\
\frac{\sqrt{3}}{2} & -\frac{1}{2}\end{array}\right)$ & $\theta_{\mathrm{r}}=\theta_{\mathrm{l}}$ & $R\left(\begin{array}{cc}\sqrt{2} & - \\
0 & V\end{array}\right.$ & $R^{2}\left(\begin{array}{cc}2 & -1 \\
-1 & 2\end{array}\right)$ & $\left(\begin{array}{cc}0 & b \\
-b & 0\end{array}\right)$ \\
\hline $\begin{array}{l}\mathbb{Z}_{3} \text {-II } \\
\text { asym. }\end{array}$ & $\left(\begin{array}{cccc}0 & 0 & 1 & 1 \\
0 & 0 & 0 & 1 \\
1 & 0 & 0 & -1 \\
-1 & 1 & 1 & 1\end{array}\right)$ & $\left(\begin{array}{ll}-\frac{1}{2} & -\frac{\sqrt{2}}{2} \\
\frac{\sqrt{3}}{2} & -\frac{1}{2}\end{array}\right.$ & $\mathbb{1}_{2}$ & $\begin{array}{l}\theta_{\mathrm{r}}^{3}=\mathbb{1}_{2} \\
\theta_{\mathrm{l}}=\mathbb{1}_{2}\end{array}$ & $\left(\begin{array}{ll}1 & -\frac{1}{2} \\
0 & \frac{\sqrt{3}}{2}\end{array}\right)$ & $\left(\begin{array}{cc}1 & -\frac{1}{2} \\
-\frac{1}{2} & 1\end{array}\right.$ & $\left(\begin{array}{cc}0 & \frac{1}{2} \\
-\frac{1}{2} & 0\end{array}\right)$ \\
\hline $\begin{array}{l}\mathbb{Z}_{3} \text {-III } \\
\text { asym. }\end{array}$ & $\left(\begin{array}{cccc}0 & 0 & -1 & 0 \\
0 & 0 & -1 & -1 \\
-1 & 1 & 1 & 1 \\
0 & -1 & -1 & 0\end{array}\right)$ & $\mathbb{1}_{2}$ & $\left(\begin{array}{cc}-\frac{1}{2} & -\frac{\sqrt{3}}{2} \\
\frac{\sqrt{3}}{2} & -\frac{1}{2}\end{array}\right)$ & $\begin{array}{l}\theta_{\mathrm{r}}=\mathbb{1}_{2} \\
\theta_{1}^{3}=\mathbb{1}_{2}\end{array}$ & $\left(\begin{array}{rr}1 & -\frac{1}{2} \\
0 & -\frac{\sqrt{2}}{2}\end{array}\right)$ & $\left(\begin{array}{cc}1 & -\frac{1}{2} \\
-\frac{1}{2} & 1\end{array}\right.$ & $\left(\begin{array}{cc}0 & \frac{1}{2} \\
-\frac{1}{2} & 0\end{array}\right)$ \\
\hline $\begin{array}{l}\mathbb{Z}_{4}-\mathrm{I} \\
\text { sym. }\end{array}$ & $\left(\begin{array}{cccc}0 & -1 & 0 & 0 \\
1 & 0 & 0 & 0 \\
0 & 0 & 0 & -1 \\
0 & 0 & 1 & 0\end{array}\right)$ & $\left(\begin{array}{cc}0 & -1 \\
1 & 0\end{array}\right)$ & $\left(\begin{array}{cc}0 & -1 \\
1 & 0\end{array}\right)$ & $\theta_{\mathrm{r}}=\theta_{\mathrm{l}}$ & $\left(\begin{array}{ll}R & 0 \\
0 & R\end{array}\right)$ & $\left(\begin{array}{cc}R^{2} & 0 \\
0 & R^{2}\end{array}\right)$ & $\left(\begin{array}{cc}0 & b \\
-b & 0\end{array}\right)$ \\
\hline $\begin{array}{l}\mathbb{Z}_{4} \text {-II } \\
\text { asym. }\end{array}$ & $\left(\begin{array}{cccc}-1 & -1 & -1 & 1 \\
0 & 0 & -1 & 1 \\
0 & 0 & 0 & -1 \\
-1 & 0 & 0 & 1\end{array}\right)$ & $\left(\begin{array}{cc}0 & -1 \\
1 & 0\end{array}\right)$ & $\left(\begin{array}{cc}-1 & 0 \\
0 & 1\end{array}\right)$ & $\begin{array}{l}\theta_{\mathrm{r}}^{4}=\mathbb{1}_{2} \\
\theta_{1}^{2}=\mathbb{1}_{2}\end{array}$ & $\left(\begin{array}{cc}\frac{1}{\sqrt{2}} & 0 \\
0 & \frac{1}{\sqrt{2}}\end{array}\right)$ & $\left(\begin{array}{cc}\frac{1}{2} & 0 \\
0 & \frac{1}{2}\end{array}\right)$ & $\left(\begin{array}{cc}0 & \frac{1}{2} \\
-\frac{1}{2} & 0\end{array}\right)$ \\
\hline $\begin{array}{l}\mathbb{Z}_{4} \text {-III } \\
\text { asym. }\end{array}$ & $\left(\begin{array}{cccc}-1 & 1 & 1 & 1 \\
0 & 0 & -1 & -1 \\
0 & 0 & 0 & 1 \\
-1 & 0 & 0 & 1\end{array}\right)$ & $\left(\begin{array}{cc}-1 & 0 \\
0 & 1\end{array}\right)$ & $\left(\begin{array}{cc}0 & -1 \\
1 & 0\end{array}\right)$ & $\begin{array}{l}\theta_{\mathrm{r}}^{2}=\mathbb{1}_{2} \\
\theta_{1}^{4}=\mathbb{1}_{2}\end{array}$ & $\left(\begin{array}{cc}\frac{1}{\sqrt{2}} & 0 \\
0 & -\frac{1}{\sqrt{2}}\end{array}\right)$ & $\left(\begin{array}{cc}\frac{1}{2} & 0 \\
0 & \frac{1}{2}\end{array}\right)$ & $\left(\begin{array}{cc}0 & \frac{1}{2} \\
-\frac{1}{2} & 0\end{array}\right)$ \\
\hline $\begin{array}{l}\mathbb{Z}_{6} \text {-I } \\
\text { sym. }\end{array}$ & $\left(\begin{array}{cccc}1 & -1 & 0 & 0 \\
1 & 0 & 0 & 0 \\
0 & 0 & 0 & -1 \\
0 & 0 & 1 & 1\end{array}\right)$ & $\left(\begin{array}{cc}\frac{1}{2} & -\frac{\sqrt{3}}{2} \\
\frac{\sqrt{3}}{2} & \frac{1}{2}\end{array}\right)$ & $\left(\begin{array}{cc}\frac{1}{2} & -\frac{\sqrt{3}}{2} \\
\frac{\sqrt{3}}{2} & \frac{1}{2}\end{array}\right)$ & $\theta_{\mathrm{r}}=\theta_{\mathrm{l}}$ & $R\left(\begin{array}{cc}\sqrt{2} & -\frac{1}{\sqrt{2}} \\
0 & \sqrt{\frac{3}{2}}\end{array}\right)$ & $R^{2}\left(\begin{array}{cc}2 & -1 \\
-1 & 2\end{array}\right)$ & $\left(\begin{array}{cc}0 & b \\
-b & 0\end{array}\right)$ \\
\hline $\begin{array}{l}\mathbb{Z}_{6} \text {-II } \\
\text { asym. }\end{array}$ & $\left(\begin{array}{cccc}0 & 0 & -1 & -1 \\
0 & 0 & 0 & -1 \\
-1 & 0 & 0 & 1 \\
1 & -1 & -1 & -1\end{array}\right)$ & $\left(\begin{array}{cc}\frac{1}{2} & -\frac{\sqrt{3}}{2} \\
\frac{\sqrt{3}}{2} & \frac{1}{2}\end{array}\right)$ & $-\mathbb{1}_{2}$ & $\begin{array}{l}\theta_{\mathrm{r}}^{6}=\mathbb{1}_{2} \\
\theta_{1}^{2}=\mathbb{1}_{2}\end{array}$ & $\left(\begin{array}{cc}1 & -\frac{1}{2} \\
0 & -\frac{\sqrt{3}}{2}\end{array}\right)$ & $\left(\begin{array}{cc}1 & -\frac{1}{2} \\
-\frac{1}{2} & 1\end{array}\right)$ & $\left(\begin{array}{cc}0 & \frac{1}{2} \\
-\frac{1}{2} & 0\end{array}\right)$ \\
\hline $\begin{array}{l}\mathbb{Z}_{6} \text {-III } \\
\text { asym. }\end{array}$ & $\left(\begin{array}{cccc}1 & -1 & 0 & 0 \\
1 & -1 & -1 & -1 \\
0 & 1 & 1 & 0 \\
0 & -1 & 0 & 0\end{array}\right)$ & $\left(\begin{array}{cc}\frac{1}{2} & -\frac{\sqrt{3}}{2} \\
\frac{\sqrt{3}}{2} & \frac{1}{2}\end{array}\right)$ & $\left(\begin{array}{cc}1 & 0 \\
0 & -1\end{array}\right)$ & $\begin{array}{l}\theta_{\mathrm{r}}^{6}=\mathbb{1}_{2} \\
\theta_{1}^{2}=\mathbb{1}_{2}\end{array}$ & $\left(\begin{array}{cc}1 & -\frac{1}{2} \\
0 & \frac{\sqrt{3}}{2}\end{array}\right)$ & $\left(\begin{array}{cc}1 & -\frac{1}{2} \\
-\frac{1}{2} & 1\end{array}\right)$ & $\left(\begin{array}{cc}0 & \frac{1}{2} \\
-\frac{1}{2} & 0\end{array}\right)$ \\
\hline $\begin{array}{l}\mathbb{Z}_{6} \text {-IV } \\
\text { asym. }\end{array}$ & $\left(\begin{array}{cccc}-1 & 0 & 0 & 1 \\
0 & 0 & 0 & 1 \\
0 & 0 & -1 & -1 \\
-1 & 1 & 1 & 1\end{array}\right)$ & $\left(\begin{array}{cc}-\frac{1}{2} & -\frac{\sqrt{3}}{2} \\
\frac{\sqrt{3}}{2} & -\frac{1}{2}\end{array}\right)$ & $\left(\begin{array}{cc}-1 & 0 \\
0 & 1\end{array}\right)$ & $\begin{array}{l}\theta_{\mathrm{r}}^{3}=\mathbb{1}_{2} \\
\theta_{1}^{2}=\mathbb{1}_{2}\end{array}$ & $\left(\begin{array}{cc}1 & -\frac{1}{2} \\
0 & \frac{\sqrt{3}}{2}\end{array}\right)$ & $\left(\begin{array}{cc}1 & -\frac{1}{2} \\
-\frac{1}{2} & 1\end{array}\right)$ & $\left(\begin{array}{cc}0 & \frac{1}{2} \\
-\frac{1}{2} & 0\end{array}\right)$ \\
\hline
\end{tabular}




\begin{tabular}{|c|c|c|c|c|c|c|c|}
\hline label & twist $\widehat{\rho}$ & twist $\theta_{\mathrm{r}}$ & twist $\theta_{1}$ & relation & vielbein $e$ & metric $G$ & $B$-field \\
\hline $\begin{array}{l}\mathbb{Z}_{6}-\mathrm{V} \\
\text { asym. }\end{array}$ & $\left(\begin{array}{cccc}-1 & 1 & 1 & 1 \\
-1 & 0 & 0 & 1 \\
1 & 0 & 0 & 0 \\
-1 & 1 & 0 & 0\end{array}\right)$ & $-\mathbb{1}_{2}$ & $\left(\begin{array}{cc}\frac{1}{2} & -\frac{\sqrt{3}}{2} \\
\frac{\sqrt{3}}{2} & \frac{1}{2}\end{array}\right)$ & $\begin{array}{l}\theta_{\mathrm{r}}^{2}=\mathbb{1}_{2} \\
\theta_{1}^{6}=\mathbb{1}_{2}\end{array}$ & $\left(\begin{array}{cc}1 & -\frac{1}{2} \\
0 & -\frac{\sqrt{3}}{2}\end{array}\right)$ & $\left(\begin{array}{cc}1 & -\frac{1}{2} \\
-\frac{1}{2} & 1\end{array}\right)$ & $\left(\begin{array}{cc}0 & \frac{1}{2} \\
-\frac{1}{2} & 0\end{array}\right)$ \\
\hline $\begin{array}{l}\mathbb{Z}_{6} \text {-VI } \\
\text { asym. }\end{array}$ & $\left(\begin{array}{cccc}0 & 0 & 0 & 1 \\
-1 & 0 & 0 & 1 \\
0 & 1 & 1 & 0 \\
0 & 0 & -1 & 0\end{array}\right)$ & $\left(\begin{array}{cc}1 & 0 \\
0 & -1\end{array}\right)$ & $\left(\begin{array}{cc}\frac{1}{2} & -\frac{\sqrt{3}}{2} \\
\frac{\sqrt{3}}{2} & \frac{1}{2}\end{array}\right)$ & $\begin{array}{l}\theta_{\mathrm{r}}^{2}=\mathbb{1}_{2} \\
\theta_{1}^{6}=\mathbb{1}_{2}\end{array}$ & $\left(\begin{array}{cc}1 & -\frac{1}{2} \\
0 & -\frac{\sqrt{3}}{2}\end{array}\right)$ & $\left(\begin{array}{cc}1 & -\frac{1}{2} \\
-\frac{1}{2} & 1\end{array}\right)$ & $\left(\begin{array}{cc}0 & \frac{1}{2} \\
-\frac{1}{2} & 0\end{array}\right)$ \\
\hline $\begin{array}{l}\mathbb{Z}_{6}-\mathrm{VII} \\
\text { asym. }\end{array}$ & $\left(\begin{array}{cccc}-1 & 1 & 0 & 0 \\
0 & 0 & -1 & -1 \\
0 & 0 & 0 & 1 \\
-1 & 0 & 0 & 0\end{array}\right)$ & $\left(\begin{array}{cc}-1 & 0 \\
0 & 1\end{array}\right)$ & $\left(\begin{array}{cc}-\frac{1}{2} & -\frac{\sqrt{3}}{2} \\
\frac{\sqrt{3}}{2} & -\frac{1}{2}\end{array}\right)$ & $\begin{array}{l}\theta_{\mathrm{r}}^{2}=\mathbb{1}_{2} \\
\theta_{1}^{3}=\mathbb{1}_{2}\end{array}$ & $\left(\begin{array}{cc}1 & -\frac{1}{2} \\
0 & -\frac{\sqrt{3}}{2}\end{array}\right)$ & $\left(\begin{array}{cc}1 & -\frac{1}{2} \\
-\frac{1}{2} & 1\end{array}\right)$ & $\left(\begin{array}{cc}0 & \frac{1}{2} \\
-\frac{1}{2} & 0\end{array}\right)$ \\
\hline $\begin{array}{l}\mathbb{Z}_{12-\mathrm{I}} \\
\text { asym. }\end{array}$ & $\left(\begin{array}{cccc}0 & 0 & 1 & 0 \\
0 & 0 & 0 & 1 \\
1 & 0 & 0 & 1 \\
0 & 1 & -1 & 0\end{array}\right)$ & $\left(\begin{array}{cc}-\frac{\sqrt{3}}{2} & \frac{1}{2} \\
-\frac{1}{2} & -\frac{\sqrt{3}}{2}\end{array}\right.$ & $\left(\begin{array}{cc}\frac{\sqrt{3}}{2} & \frac{1}{2} \\
-\frac{1}{2} & \frac{\sqrt{3}}{2}\end{array}\right)$ & $\theta_{\mathrm{l}}=\theta_{\mathrm{r}}^{5}$ & $\left(\begin{array}{cc}\frac{3^{\frac{1}{4}}}{\sqrt{2}} & 0 \\
0 & \frac{3^{\frac{1}{4}}}{\sqrt{2}}\end{array}\right)$ & $\left(\begin{array}{cc}\frac{\sqrt{3}}{2} & 0 \\
0 & \frac{\sqrt{3}}{2}\end{array}\right)$ & $\left(\begin{array}{cc}0 & -\frac{1}{2} \\
\frac{1}{2} & 0\end{array}\right)$ \\
\hline
\end{tabular}

Table 3: This table presents a large number of examples for $\mathbb{Z}_{K}$ Narain orbifolds in two dimensions. For each inequivalent orbifold it gives important data that characterizes Narain orbifolds, like the twists in both, the lattice and the coordinate basis and the values of the (frozen) moduli.

from eq. (8.5). Then we follow the procedure outlined in section 5.3 to find that all Narain moduli are stabilized and take the form

$$
e_{(1)}=\frac{3^{\frac{1}{4}}}{\sqrt{2}} \mathbb{1}_{2} \quad \text { and } \quad B_{(1)}=-\frac{1}{2} \epsilon,
$$

while the twist $\Theta_{(1)}$ is given by

$$
\theta_{(1) \mathrm{r}}=\left(\begin{array}{cc}
-\frac{\sqrt{3}}{2} & \frac{1}{2} \\
-\frac{1}{2} & -\frac{\sqrt{3}}{2}
\end{array}\right) \quad \text { and } \quad \theta_{(1) \mathrm{l}}=\theta_{(1) \mathrm{r}}^{5} .
$$

This precisely corresponds to the data given for the $\mathbb{Z}_{12}$-I orbifold in table 3 .

An equivalent description of this asymmetric $\mathbb{Z}_{12}$-I orbifold has no $B$-field at all $\left(B_{(2)}=0\right)$. For this case we take

$$
\widehat{\rho}_{(2)}=\left(\begin{array}{cccc}
0 & 0 & 1 & 1 \\
0 & 0 & 0 & 1 \\
1 & 0 & 0 & 0 \\
-1 & 1 & 0 & 0
\end{array}\right) \in \mathrm{O}_{\widehat{\eta}}(2,2 ; \mathbb{Z}) .
$$

The stabilized Narain moduli are now given by

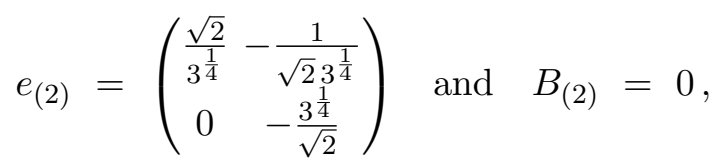

with the twist $\Theta_{(2)}$ is given by

$$
\theta_{(2) \mathrm{r}}=\left(\begin{array}{cc}
-\frac{\sqrt{3}}{2} & \frac{1}{2} \\
-\frac{1}{2} & -\frac{\sqrt{3}}{2}
\end{array}\right) \quad \text { and } \quad \theta_{(2) 1}=\theta_{\mathrm{r}}^{7}=-\theta_{\mathrm{r}} .
$$


To show explicitly that these two $\mathbb{Z}_{12}$ orbifolds are $\mathbb{Z}$-equivalent (and consequently also $\mathbb{Q}$-equivalent), we observe that we can relate the two $\mathbb{Z}_{12}$ generators,

$$
\widehat{M} \widehat{\rho}_{(2)}=\widehat{\rho}_{(1)} \widehat{M}
$$

using the matrix

$$
\widehat{M}=\left(\begin{array}{cccc}
-1 & 0 & 0 & 0 \\
0 & 0 & 0 & -1 \\
0 & 0 & -1 & -1 \\
1 & -1 & 0 & 0
\end{array}\right) \in \mathrm{O}_{\widehat{\eta}}(2,2 ; \mathbb{Z})
$$

Here, we used that both generators $\widehat{\rho}_{(1)}$ and $\widehat{\rho}_{(2)}$ are defined with respect to the same Narain metric $\widehat{\eta}$. Hence, the corresponding Narain point groups $\widehat{\mathbf{P}}_{(1)}$ and $\widehat{\mathbf{P}}_{(2)}$ are identical up to the discrete $T$-duality transformation with $\widehat{M}$, i.e. these point groups lie in the same $\mathbb{Z}$ class. In other words, we have described the same asymmetric $\mathbb{Z}_{12}$ orbifold in two different duality frames, once with and once without $B$-field.

\subsection{Exposing a seemingly asymmetric $\mathbb{Z}_{3}$ Narain orbifold}

It might happen that one uses a description, i.e. choice of duality frame, in which a given Narain orbifold appears to be asymmetric. Consider for example a two-dimensional $\mathbb{Z}_{3}$ Narain orbifold defined by the twist

$$
\widehat{\rho}_{(\mathrm{a})}=\left(\begin{array}{cc}
0 & \epsilon \\
\epsilon & -\mathbb{1}_{2}
\end{array}\right) \in \mathrm{O}_{\widehat{\eta}}(2,2 ; \mathbb{Z}),
$$

in the lattice basis. We use the subscript (a) to refer to this seemingly asymmetric orbifold: it is not obviously a symmetric orbifold, as it does not meet the sufficient condition $(\widehat{\rho})_{12}=0$ for being a symmetric Narain orbifold formulated in section 5.3. Since in this case, eqs. (8.5) reduce to

$$
\widehat{M}_{(\mathrm{a}) 1}=-\epsilon, \quad \widehat{M}_{(\mathrm{a}) 2}=\mathbb{1}_{2}+\left(G_{(\mathrm{a})}-B_{(\mathrm{a})}\right) \epsilon,
$$

the Narain moduli are given by

$$
e_{(\mathrm{a})}=\left(\begin{array}{cc}
R_{(\mathrm{a})} & w_{(\mathrm{a})} \\
0 & -\frac{\sqrt{3}}{2 R_{(\mathrm{a})}}
\end{array}\right) \text { and } B_{(\mathrm{a})}=-\frac{1}{2} \epsilon,
$$

where parameters $R_{(\mathrm{a})}$ and $w_{(\mathrm{a})}$ are unconstrained. Furthermore, the twist $\Theta_{(\mathrm{a})}$ is specified by

$$
\theta_{(\mathrm{a}) \mathrm{r}}=\left(\begin{array}{cc}
-\frac{1}{2} & \frac{\sqrt{3}}{2} \\
-\frac{\sqrt{3}}{2} & -\frac{1}{2}
\end{array}\right) \quad \text { and } \quad \theta_{(\mathrm{a}) \mathrm{l}}=\theta_{(\mathrm{a}) \mathrm{r}}^{2} .
$$

Since $\theta_{(\mathrm{a}) \mathrm{r}} \neq \theta_{(\mathrm{a}) \mathrm{l}}$, this seems to indicate that this an asymmetric Narain orbifold. However, it is equivalent to the symmetric orbifold $\mathbb{Z}_{3}$-I of table 3 : to see this, we describe this symmetric $\mathbb{Z}_{3}$-I orbifold (labelled with a subscript (s)) in some detail: the defining twist in the lattice basis is given by

$$
\widehat{\rho}_{(\mathrm{s})}=\left(\begin{array}{cccc}
0 & -1 & 0 & 0 \\
1 & -1 & 0 & 0 \\
0 & 0 & -1 & -1 \\
0 & 0 & 1 & 0
\end{array}\right) \in \mathrm{O}_{\widehat{\eta}}(2,2 ; \mathbb{Z})
$$


from which we obtain

$$
\begin{array}{ll}
\widehat{M}_{(\mathrm{s}) 1}=\left(G_{(\mathrm{s})}-B_{(\mathrm{s})}\right) \rho_{(\mathrm{s}) \mathrm{r}}, & \widehat{M}_{(\mathrm{s}) 2}=-\left(\rho_{(\mathrm{s}) \mathrm{r}}\right)^{-T} \\
\rho_{(\mathrm{s}) \mathrm{r}} & =e_{(\mathrm{s})}^{-1} \theta_{(\mathrm{s}) \mathrm{r}} e_{(\mathrm{s})}=\left(\widehat{\rho}_{(\mathrm{s})}\right)_{11}=\left(\begin{array}{c}
0-1 \\
1-1
\end{array}\right) .
\end{array}
$$

In this case, $\rho_{(\mathrm{s}) \mathrm{r}}$ acts cryptographically on $e$, i.e. the first column $e_{1}$ of $e$ is mapped to the second column $e_{2}$ and $e_{2}$ is mapped to $-e_{1}-e_{2}$. Furthermore, the Narain moduli are given by

$$
e_{(\mathrm{s})}=R_{(\mathrm{s})}\left(\begin{array}{cc}
\sqrt{2} & -\frac{1}{\sqrt{2}} \\
0 & \sqrt{\frac{3}{2}}
\end{array}\right) \Rightarrow G_{(\mathrm{s})}=R_{(\mathrm{s})}^{2}\left(\begin{array}{cc}
2 & -1 \\
-1 & 2
\end{array}\right) \quad \text { and } \quad B_{(\mathrm{s})}=b_{(\mathrm{s})}\left(\begin{array}{cc}
0 & 1 \\
-1 & 0
\end{array}\right)
$$

where $R_{(\mathrm{s})}$ and $b_{(\mathrm{s})}$ are unconstrained. Thus, the vielbein $e_{(\mathrm{s})}$ spans the root lattice of $\mathrm{SU}(3)$ multiplied by an arbitrary radius $R_{(\mathrm{s})}$. Furthermore, the twist $\Theta_{(\mathrm{s})}$ is specified by

$$
\theta_{(\mathrm{s}) \mathrm{r}}=\theta_{(\mathrm{s}) \mathrm{l}}=\left(\begin{array}{cc}
-\frac{1}{2} & -\frac{\sqrt{3}}{2} \\
\frac{\sqrt{3}}{2} & -\frac{1}{2}
\end{array}\right) \text {. }
$$

Clearly, these two descriptions look very different: the parametrization of the moduli does not seem to be alike, since, for example, in case (a) the $B$-field is fixed while in case (s) it is a modulus. Moreover, the twist seems to be asymmetric for case (a) but symmetric for case (s). However, their Narain point groups $\widehat{\mathbf{P}}_{(\mathrm{s})}$ and $\widehat{\mathbf{P}}_{(\mathrm{a})}$ belong to the same $\mathbb{Z}$-class (and consequently also to the same $\mathbb{Q}$-class); they are equivalent up to a discrete $T$-duality transformation.

Explicitly, the discrete $T$-duality transformation that relates $\widehat{\mathbf{P}}_{(\mathrm{s})}$ and $\widehat{\mathbf{P}}_{(\mathrm{a})}$ reads

$$
\widehat{M}=\left(\begin{array}{cccc}
-1 & -1 & 0 & 0 \\
0 & 0 & -1 & 1 \\
0 & 0 & -1 & 0 \\
0 & 1 & 0 & 0
\end{array}\right) \in \mathrm{O}_{\widehat{\eta}}(2,2 ; \mathbb{Z}) \quad \text { with } \quad \widehat{M} \widehat{\rho}_{(\mathrm{a})}=\widehat{\rho}_{(\mathrm{s})} \widehat{M}
$$

where we used that $\widehat{\rho}_{(\mathrm{s})}$ and $\widehat{\rho}_{(\mathrm{a})}$ are both defined with respect to the same Narain metric $\widehat{\eta}$. This implies that also the moduli $\left(R_{(\mathrm{s})}, b_{(\mathrm{s})}\right)$ and $\left(R_{(\mathrm{a})}, w_{(\mathrm{a})}\right)$ can be mapped explicitly by exploiting the transformation formula (8.7): we Use

$$
\widehat{U}_{\widehat{M}} \widehat{E}\left(e_{(\mathrm{a})}, B_{(\mathrm{a})}\right)=\widehat{E}\left(e_{(\mathrm{s})}, B_{(\mathrm{s})}\right) \widehat{M}
$$

with $\widehat{M}$ given in eq. (8.27) to relate the moduli in both descriptions as

$$
\begin{aligned}
G_{(\mathrm{a})} & =\frac{1}{2 R_{(\mathrm{s})}^{2}}\left(\begin{array}{cc}
b_{(\mathrm{s})}^{2}+3 R_{(\mathrm{s})}^{4} & b_{(\mathrm{s})}+b_{(\mathrm{s})}^{2}+3 R_{(\mathrm{s})}^{4} \\
b_{(\mathrm{s})}+b_{(\mathrm{s})}^{2}+3 R_{(\mathrm{s})}^{4} & \left(1+b_{(\mathrm{s})}\right)^{2}+3 R_{(\mathrm{s})}^{4}
\end{array}\right), \\
B_{(\mathrm{a})} & =-\frac{1}{2} \epsilon
\end{aligned}
$$


This results in

$$
w_{(\mathrm{a})}=\frac{1}{\sqrt{2} R_{(\mathrm{s})}} \frac{b_{(\mathrm{s})}+b_{(\mathrm{s})}^{2}+3 R_{(\mathrm{s})}^{4}}{\sqrt{b_{(\mathrm{s})}^{2}+3 R_{(\mathrm{s})}^{4}}} \text { and } \quad R_{(\mathrm{a})}=\frac{1}{\sqrt{2} R_{(\mathrm{s})}} \sqrt{b_{(\mathrm{s})}^{2}+3 R_{(\mathrm{s})}^{4}} .
$$

In addition, we compute $u_{\mathrm{r}}$ and $u_{\mathrm{l}}$ from eqs. (8.6) and (8.7) to obtain

$$
u_{\mathrm{l}}=\left(\begin{array}{cc}
\frac{1}{2} & \frac{\sqrt{3}}{2} \\
\frac{\sqrt{3}}{2} & -\frac{1}{2}
\end{array}\right) u_{\mathrm{r}} ; \quad u_{\mathrm{r}}=\frac{1}{2 \sqrt{b_{(\mathrm{s})}^{2}+3 R_{(\mathrm{s})}^{4}}}\left(\begin{array}{cc}
b_{(\mathrm{s})}-3 R_{(\mathrm{s})}^{2} & -\sqrt{3}\left(b_{(\mathrm{s})}+R_{(\mathrm{s})}^{2}\right) \\
-\sqrt{3}\left(b_{(\mathrm{s})}+R_{(\mathrm{s})}^{2}\right) & -b_{(\mathrm{s})}+3 R_{(\mathrm{s})}^{2}
\end{array}\right) .
$$

Note that $\operatorname{det}\left(u_{\mathrm{l}}\right)=+1$ but $\operatorname{det}\left(u_{\mathrm{r}}\right)=-1$. This corresponds to the matrix $U_{\mathrm{B}}$ from eq. (6.8) that maps the symmetric twist from point group $\mathbf{P}_{(\mathrm{s})}$ to the seemingly asymmetric twist from point group $\mathbf{P}_{(\mathrm{a})}$.

Let us close this subsection with the comment that for Narain orbifolds of order 3 , we were able to distinguish between three $\mathbb{Q}$-classes, where each $\mathbb{Q}$-class contains only a single $\mathbb{Z}$-class. In the nomenclature of table 3 the two-dimensional Narain orbifold $\mathbb{Z}_{3}$-I is a symmetric orbifold, while the other two, $\mathbb{Z}_{3}$-II and $\mathbb{Z}_{3}$-II, are asymmetric. In fact, they are each others mirrors in the sense that their $\theta_{1}$ and $\theta_{\mathrm{r}}$ are interchanged.

\subsection{Symmetric $\mathbb{Z}_{2}$ Narain orbifolds from inequivalent $\mathbb{Z}$-classes}

For the examples considered so far, we found that each Narain $\mathbb{Q}$-class contains just a single Narain $\mathbb{Z}$-class. This might convey the impression that the notion of $\mathbb{Z}$-classes for Narain orbifolds is obsolete. To emphasize that this is not the case, we consider two symmetric $\mathbb{Z}_{2}$ Narain point groups in $D=2$ next. Both correspond geometrically to the Möbius strip, where the $B$-field is either turned on or off. We will show that even though these two Narain point groups belong to the same Narain Q-class, they live in different Narain $\mathbb{Z}$-classes, hence they are physically inequivalent.

Consider the symmetric $\mathbb{Z}_{2}$-II-2 Narain orbifold of table 3 without a $B$-field: in detail, we choose

$$
\widehat{\rho}_{(1)}=\left(\begin{array}{llll}
0 & 1 & 0 & 0 \\
1 & 0 & 0 & 0 \\
0 & 0 & 0 & 1 \\
0 & 0 & 1 & 0
\end{array}\right) \in \mathrm{O}_{\widehat{\eta}}(2,2 ; \mathbb{Z})
$$

and obtain

$$
\widehat{M}_{(1) 1}=\left(G_{(1)}-B_{(1)}\right)\left(\begin{array}{ll}
0 & 1 \\
1 & 0
\end{array}\right) \quad \text { and } \quad \widehat{M}_{(1) 2}=-\left(\begin{array}{ll}
0 & 1 \\
1 & 0
\end{array}\right) .
$$

In this case, the Narain moduli are given by

$$
e_{(1)}=\left(\begin{array}{cc}
R_{1} & R_{1} \\
-R_{2} & R_{2}
\end{array}\right) \Rightarrow G_{(1)}=\left(\begin{array}{cc}
R_{1}^{2}+R_{2}^{2} & R_{1}^{2}-R_{2}^{2} \\
R_{1}^{2}-R_{2}^{2} & R_{1}^{2}+R_{2}^{2}
\end{array}\right) \quad \text { and } \quad B_{(1)}=\left(\begin{array}{ll}
0 & 0 \\
0 & 0
\end{array}\right),
$$

for $R_{1} R_{2} \neq 0$. Furthermore, the twist $\Theta$ is specified by

$$
\theta_{\mathrm{r}}=\theta_{\mathrm{l}}=\left(\begin{array}{cc}
1 & 0 \\
0 & -1
\end{array}\right)
$$




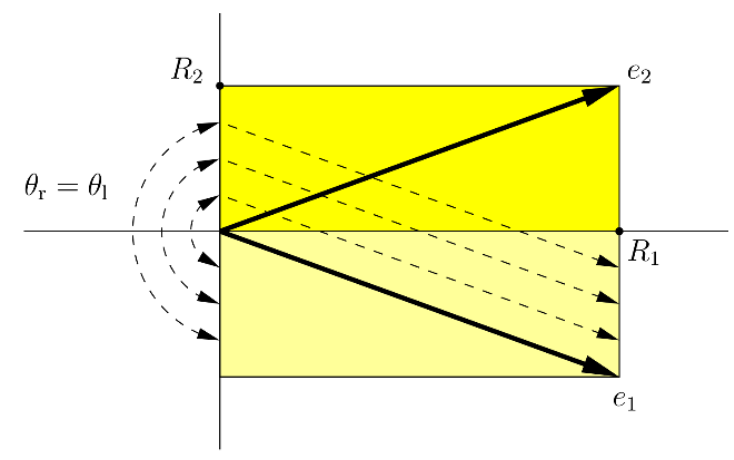

Figure 1. Two-dimensional Möbius strip as $\mathbb{Z}_{2}$ orbifold: the underlying two-torus lattice is spanned by $e_{1}$ and $e_{2}$. The upper and lower yellow regions combined give a convenient choice for the fundamental domain of the resulting two-torus. The symmetric twist $\theta_{\mathrm{r}}=\theta_{1}$ gives a reflection at the horizontal axis. Consequently, we may take the lower yellow region to represent a fundamental domain of the resulting $\mathbb{Z}_{2}$ orbifold. In this picture the $3+3$ dashed arrows illustrate how the left and right side of the lower yellow region get glued together, hence this orbifold corresponds to the Möbius strip.

This orbifold geometrically corresponds to the Möbius strip, see figure 1.

Another symmetric $\mathbb{Z}_{2}$ orbifold has a non-vanishing $B$-field: for this $\mathbb{Z}_{2}$-II-3 Narain orbifold in table 3 we choose

$$
\widehat{\rho}_{(2)}=\left(\begin{array}{cccc}
0 & 1 & 0 & 0 \\
1 & 0 & 0 & 0 \\
-1 & 0 & 0 & 1 \\
0 & 1 & 1 & 0
\end{array}\right)=\widehat{M}_{B}(\Delta B)^{-1} \widehat{\rho}_{(1)} \widehat{M}_{B}(\Delta B) \in \mathrm{O}_{\widehat{\eta}}(2,2 ; \mathbb{Z}),
$$

where $\widehat{\rho}_{(1)}$ is the twist from the $\mathbb{Z}_{2}$ orbifold discussed just above and $\widehat{M}_{B}(\Delta B)$ is a fractional $B$-field shift given by

$$
\widehat{M}_{B}(\Delta B)=\left(\begin{array}{cc}
\mathbb{1}_{2} & 0 \\
\Delta B & \mathbb{1}_{2}
\end{array}\right) \quad \text { for } \quad \Delta B=\frac{1}{2} \epsilon .
$$

Now, we obtain

$$
\widehat{M}_{(2) 1}=\left(\begin{array}{cc}
1 & 0 \\
0 & -1
\end{array}\right)+(G-B)\left(\begin{array}{ll}
0 & 1 \\
1 & 0
\end{array}\right) \quad \text { and } \quad \widehat{M}_{(2) 2}=-\left(\begin{array}{ll}
0 & 1 \\
1 & 0
\end{array}\right) .
$$

In this case, the Narain moduli are given by

$$
e_{(2)}=\left(\begin{array}{cc}
R_{1} & R_{1} \\
-R_{2} & R_{2}
\end{array}\right) \Rightarrow G_{(2)}=\left(\begin{array}{cc}
R_{1}^{2}+R_{2}^{2} & R_{1}^{2}-R_{2}^{2} \\
R_{1}^{2}-R_{2}^{2} & R_{1}^{2}+R_{2}^{2}
\end{array}\right) \quad \text { and } \quad B_{(2)}=\left(\begin{array}{cc}
0 & \frac{1}{2} \\
-\frac{1}{2} & 0
\end{array}\right),
$$

for $R_{1} R_{2} \neq 0$. Furthermore, the twist $\Theta$ remains unchanged, i.e.

$$
\theta_{\mathrm{r}}=\theta_{\mathrm{l}}=\left(\begin{array}{cc}
1 & 0 \\
0 & -1
\end{array}\right)
$$


Note, that the metric $G_{(2)}$ is identical to $G_{(1)}$ from the case above; the only difference is that we now have a non-vanishing $B$-field.

The conjugation of the generator $\widehat{\rho}_{(1)}$ with $\widehat{M}_{B}(\Delta B)$ in eq. (8.36) tells us that these two Narain point groups belong to the same $\mathbb{Q}$-class. However, it turns out that they are from different $\mathbb{Z}$-classes: there is no $\widehat{M} \in \mathrm{O}_{\widehat{\eta}}(D, D ; \mathbb{Z})$ that can relate $\widehat{\rho}_{(1)}$ to $\widehat{\rho}_{(2)}$. Since the transformation (8.36) is a conjugation with a discrete fractional $B$-field transformation, the $\mathbb{Z}$-classes under investigation can be used to parametrize the inequivalent choices for the $B$-field for the given geometrical setting. As can be inferred from table 3 we identified three inequivalent $\mathbb{Z}$-classes for the $\mathbb{Q}$-class $\mathbb{Z}_{2}$-II, where $\mathbb{Z}_{2}$-II-1 and $\mathbb{Z}_{2}$-II-2 both have vanishing $B$-field but are based on inequivalent lattices.

\section{Acknowledgments}

This work was partially supported by the DFG cluster of excellence "Origin and Structure of the Universe" (www.universe-cluster.de) and by the Deutsche Forschungsgemeinschaft (SFB1258).

\section{A Moduli deformations and the generalized metric}

Choose a specific generalized metric $\mathcal{H}_{0}$. Next, consider the finite group of all discrete $T$ duality transformations that leaves this generalized metric invariant and choose a subgroup $\widehat{\mathbf{H}}$ thereof. Then, the general question, which we are addressing in this section, reads: what infinitesimal moduli deformations are allowed such that the deformed generalized metric stays invariant under all transformations from $\widehat{\mathbf{H}}$ ? We will answer this question in three steps. First, we define the group $\widehat{\mathbf{H}}$ in appendix A.1. Second, we parametrize all infinitesimal moduli deformations in appendix A.2. Third, in appendix A.3 we restrict them to the ones which are compatible with the action of $\widehat{\mathbf{H}}$. In addition, in appendix A.4 we derive a closed expression which counts the number of moduli that are compatible with the action of $\widehat{\mathbf{H}}$. We use the results form this appendix in section 5.4 , where we set $\widehat{\mathbf{H}}=\widehat{\mathbf{P}}$, i.e. equal to the point group in the lattice basis. By doing so, we identify the moduli in Narain orbifolds.

\section{A.1 T-duality transformations that leave a generalized metric invariant}

Consider a subgroup $\widehat{\mathbf{H}}$ of the group of all $\mathrm{O}_{\hat{\eta}}(D, D+16 ; \mathbb{Z})$ transformations which leave a specific generalized metric $\mathcal{H}_{0}=E_{0}^{T} E_{0}$ invariant, i.e.

$$
\widehat{\mathbf{H}} \subseteq\left\{\widehat{M} \in \mathrm{O}_{\hat{\eta}}(D, D+16 ; \mathbb{Z}) \mid \widehat{M}^{T} \mathcal{H}_{0} \widehat{M}=\mathcal{H}_{0}\right\}
$$

The following discussion is independent of whether $\widehat{\mathbf{H}}$ is Abelian or non-Abelian. Since the elements $\widehat{M} \in \widehat{\mathbf{H}}$ preserve both $\hat{\eta}$ and $\mathcal{H}_{0}$ we find that the corresponding element $\Theta(\widehat{M})$ as a function of $\widehat{M}$ is given by

$$
\Theta(\widehat{M})=E_{0} \widehat{M} E_{0}^{-1} \quad \text { with } \quad \Theta(\widehat{M})^{T} \Theta(\widehat{M})=\mathbb{1}, \quad \Theta(\widehat{M})^{T} \eta \Theta(\widehat{M})=\eta
$$


Hence,

$$
\Theta(\widehat{M})=\left(\begin{array}{cc}
\theta_{\mathrm{r}}(\widehat{M}) & 0 \\
0 & \Theta_{\mathrm{L}}(\widehat{M})
\end{array}\right) \subset \mathrm{O}(D ; \mathbb{R}) \times \mathrm{O}(D+16 ; \mathbb{R})
$$

and $\Theta(\widehat{M})$ is a group homomorphism from $\widehat{\mathbf{H}}$ to a finite subgroup of $\mathrm{O}(D ; \mathbb{R}) \times \mathrm{O}(D+16 ; \mathbb{R})$.

\section{A.2 Infinitesimal moduli deformations of the Narain lattice}

We want to determine which parameters $\delta E$ in the generalized vielbein can be deformed infinitesimally, i.e. $E_{0} \rightarrow E_{0}+\delta E$ to first order in the perturbations. Since the generalized vielbein with $(2 D+16)^{2}$ components is parametrized in terms of $D(D+16)$ parameters (i.e. the vielbein $e$, the $B$-field and the Wilson lines $A$ ), not all components of $\delta E$ are independent. To characterize the infinitesimal moduli perturbations without choosing a particular parametrization, we expand the constraint $E_{0}^{T} \eta E_{0}=\hat{\eta}$ from eq. (2.17) to first order in $\delta E$ and obtain

$$
\delta E^{T} \eta E_{0}+E_{0}^{T} \eta \delta E=0 .
$$

This can be cast into the form

$$
\delta \mathfrak{e}^{T} \eta+\eta \delta \mathfrak{e}=0
$$

where we have defined $\delta \mathfrak{e}=\delta E E_{0}^{-1}$. The general solution reads

$$
\delta \mathfrak{e}=\delta E E_{0}^{-1}=\frac{1}{2}\left(\begin{array}{cc}
\delta \mathfrak{u}_{D} & \delta \mathfrak{m} \\
\delta \mathfrak{m}^{T} & \delta \mathfrak{u}_{D+16}
\end{array}\right),
$$

with $\delta \mathfrak{m} \in M_{D \times(D+16)}(\mathbb{R})$. Furthermore, $\delta \mathfrak{u}_{D}^{T}=-\delta \mathfrak{u}_{D}$ and $\delta \mathfrak{u}_{D+16}^{T}=-\delta \mathfrak{u}_{D+16}$ generate $O(D ; \mathbb{R})$ and $O(D+16 ; \mathbb{R})$, respectively. These orthogonal groups correspond to the $U$ transformation in eq. (2.40). Next, we consider the perturbations of the generalized metric $\delta \mathcal{H}=\delta E^{T} E_{0}+E_{0}^{T} \delta E$ to first order. Using eq. (A.4) one can see that the constraint $\left(\hat{\eta}^{-1}\left(\mathcal{H}_{0}+\delta \mathcal{H}\right)\right)^{2}=\mathbb{1}$ from eq. (5.7) is fulfilled. In fact, we may write $\delta \mathfrak{h}=\delta \mathfrak{e}^{T}+\delta \mathfrak{e}$, where $\delta \mathfrak{e}=\frac{1}{2} \delta \mathfrak{h}+\frac{1}{2} \delta \mathfrak{u}$ with

$$
\delta \mathfrak{u}=-\delta \mathfrak{u}^{T}=\left(\begin{array}{cc}
\delta \mathfrak{u}_{D} & 0 \\
0 & \delta \mathfrak{u}_{D+16}
\end{array}\right) .
$$

Hence, the infinitesimal moduli are uniquely identified by $\delta \mathfrak{m}$, i.e. $\delta \mathfrak{m}$ encodes the deformations of the metric $\delta G$, the $B$-field $\delta B$ and the Wilson lines $\delta A$. This can be stated explicitly as follows. We can determine $\delta \mathfrak{e}$ by using eq. (A.6) with $E_{0}=R \widehat{E}$ and the expression for $\hat{E}$ given in eq. (2.39). Thereby we directly confirm that $\delta \mathfrak{u}_{D}$ and $\delta \mathfrak{u}_{D+16}$ are anti-symmetric and we derive that $\delta \mathfrak{m}$ is given at linear order in the moduli perturbations $\delta G, \delta B$ and $\delta A$ as given in eq. (5.21), using $\left(e_{0}+\delta e\right)^{-1} \approx e^{-1}-e_{0}^{-1} \delta e e_{0}^{-1}$.

\section{A.3 $\widehat{H}$-invariant infinitesimal moduli deformations}

In order to determine which of the Narain moduli are compatible with the action of $\widehat{\mathbf{H}}$ we consider the first order perturbation of eq. (A.1) and obtain

$$
\widehat{M}^{T} \delta \mathcal{H} \widehat{M}=\delta \mathcal{H} \quad \Leftrightarrow \quad \Theta(\widehat{M})^{T} \delta \mathfrak{h} \Theta(\widehat{M})=\delta \mathfrak{h} .
$$


This reads on the level of the moduli deformations

$$
\theta_{\mathrm{r}}^{T}(\widehat{M}) \delta \mathfrak{m} \Theta_{\mathrm{L}}(\widehat{M})=\delta \mathfrak{m}
$$

for each $\widehat{M} \in \widehat{\mathbf{H}}$. Eq. (A.9) can be written as

$$
\left(\theta_{\mathrm{r}}(\widehat{M}) \otimes \Theta_{\mathrm{L}}(\widehat{M})\right) \delta \mathfrak{m}=\delta \mathfrak{m}
$$

Here, we interpret $\delta \mathfrak{m}$ as a vector with $D(D+16)$ components using the standard tensor product notation $\otimes$. To solve this condition we introduce the projection operator $\mathcal{P}_{\widehat{\mathbf{H}}}$ that projects the moduli perturbations on their $\widehat{\mathbf{H}}$-invariant subspace, i.e.

$$
\mathcal{P}_{\widehat{\mathbf{H}}}=\frac{1}{|\widehat{\mathbf{H}}|} \sum_{\widehat{M} \in \widehat{\mathbf{H}}} \theta_{\mathrm{r}}(\widehat{M}) \otimes \Theta_{\mathrm{L}}(\widehat{M}) \quad \text { with } \quad\left(\theta_{\mathrm{r}}(\widehat{M}) \otimes \Theta_{\mathrm{L}}(\widehat{M})\right) \mathcal{P}_{\widehat{\mathbf{H}}}=\mathcal{P}_{\widehat{\mathbf{H}}}
$$

Using that $\Theta(\widehat{M})$ defines a group homomorphism, it is not difficult to show that this indeed defines a projection operator, i.e. $\mathcal{P}_{\widehat{\mathbf{H}}}^{2}=\mathcal{P}_{\widehat{\mathbf{H}}}$. Thus, the $\widehat{\mathbf{H}}$-invariant moduli space is given by

$$
\mathcal{M}_{\widehat{\mathbf{H}}}=\left\{\delta \mathfrak{m}_{\widehat{\mathbf{H}}}=\mathcal{P}_{\widehat{\mathbf{H}}} \delta \mathfrak{m}\right\}
$$

\section{A.4 The number of $\widehat{\mathbf{H}}$-invariant Narain moduli}

The dimension of the $\widehat{\mathbf{H}}$-invariant Narain moduli space is determined by the trace of the projection operator $\mathcal{P}_{\widehat{\mathbf{H}}}$, i.e.

$$
\begin{aligned}
\operatorname{dim}\left(\mathcal{M}_{\widehat{\mathbf{H}}}\right) & =\operatorname{tr}\left(\mathcal{P}_{\widehat{\mathbf{H}}}\right)=\frac{1}{|\widehat{\mathbf{H}}|} \sum_{\widehat{M} \in \widehat{\mathbf{H}}} \chi\left(\theta_{\mathrm{r}}(\widehat{M})\right) \chi\left(\Theta_{\mathrm{L}}(\widehat{M})\right)^{*} \\
& =\frac{1}{|\mathbf{H}|} \sum_{\Theta \in \mathbf{H}} \chi_{\mathrm{r}}(\Theta) \chi_{\mathrm{L}}(\Theta)^{*}
\end{aligned}
$$

Here, we have used the linearity of the $\operatorname{trace}, \operatorname{tr}(A \otimes B)=\operatorname{tr}(A) \operatorname{tr}(B)$ and we have used the definition (5.7). In addition, we have included a complex conjugate in eq. (A.13) for later use. Furthermore, we have introduced the group characters

$$
\begin{aligned}
& \chi_{\mathrm{r}}(\Theta)=\chi\left(\theta_{\mathrm{r}}(\widehat{M})\right)=\operatorname{tr}\left(\theta_{\mathrm{r}}(\widehat{M})\right)=\operatorname{tr}\left[\frac{\mathbb{1}-\mathcal{Z}}{2} \widehat{M}\right] \\
& \chi_{\mathrm{L}}(\Theta)=\chi\left(\Theta_{\mathrm{L}}(\widehat{M})\right)=\operatorname{tr}\left(\Theta_{\mathrm{L}}(\widehat{M})\right)=\operatorname{tr}\left[\frac{\mathbb{1}+\mathcal{Z}}{2} \widehat{M}\right]
\end{aligned}
$$

which are real for the real representations $\theta_{\mathrm{r}}(\widehat{M})$ and $\Theta_{\mathrm{L}}(\widehat{M})$, respectively.

Open Access. This article is distributed under the terms of the Creative Commons Attribution License (CC-BY 4.0), which permits any use, distribution and reproduction in any medium, provided the original author(s) and source are credited. 


\section{References}

[1] D.J. Gross, J.A. Harvey, E.J. Martinec and R. Rohm, The heterotic string, Phys. Rev. Lett. 54 (1985) 502 [INSPIRE].

[2] D.J. Gross, J.A. Harvey, E.J. Martinec and R. Rohm, Heterotic string theory. 1. The free heterotic string, Nucl. Phys. B 256 (1985) 253 [INSPIRE].

[3] D.J. Gross, J.A. Harvey, E.J. Martinec and R. Rohm, Heterotic string theory. 2. The interacting heterotic string, Nucl. Phys. B 267 (1986) 75 [InSPIRE].

[4] L.E. Ibáñez and A.M. Uranga, String theory and particle physics: an introduction to string phenomenology, Cambridge University Press, Cambridge U.K., (2012) [INSPIRE].

[5] L.J. Dixon, J.A. Harvey, C. Vafa and E. Witten, Strings on orbifolds, Nucl. Phys. B 261 (1985) 678 [INSPIRE].

[6] L.J. Dixon, J.A. Harvey, C. Vafa and E. Witten, Strings on orbifolds. 2, Nucl. Phys. B 274 (1986) 285 [INSPIRE].

[7] M. Fischer, M. Ratz, J. Torrado and P.K.S. Vaudrevange, Classification of symmetric toroidal orbifolds, JHEP 01 (2013) 084 [arXiv:1209.3906] [INSPIRE].

[8] L.E. Ibáñez, H.P. Nilles and F. Quevedo, Orbifolds and Wilson lines, Phys. Lett. B 187 (1987) 25 [INSPIRE].

[9] L.E. Ibáñez, J.E. Kim, H.P. Nilles and F. Quevedo, Orbifold compactifications with three families of $\mathrm{SU}(3) \times \mathrm{SU}(2) \times \mathrm{U}(1)^{n}$, Phys. Lett. B 191 (1987) 282 [INSPIRE].

[10] J.A. Casas and C. Muñoz, Three generation $\mathrm{SU}(3) \times \mathrm{SU}(2) \times \mathrm{U}(1)_{Y}$ models from orbifolds, Phys. Lett. B 214 (1988) 63 [INSPIRE].

[11] J.A. Casas, E.K. Katehou and C. Muñoz, U(1) charges in orbifolds: anomaly cancellation and phenomenological consequences, Nucl. Phys. B 317 (1989) 171 [INSPIRE].

[12] A. Font, L.E. Ibáñez, F. Quevedo and A. Sierra, The construction of 'realistic' four-dimensional strings through orbifolds, Nucl. Phys. B 331 (1990) 421 [InSPIRE].

[13] D. Bailin and A. Love, Orbifold compactifications of string theory, Phys. Rept. 315 (1999) 285 [INSPIRE].

[14] S. Förste, H.P. Nilles, P.K.S. Vaudrevange and A. Wingerter, Heterotic brane world, Phys. Rev. D 70 (2004) 106008 [hep-th/0406208] [INSPIRE].

[15] T. Kobayashi, S. Raby and R.-J. Zhang, Searching for realistic 4d string models with a Pati-Salam symmetry: orbifold grand unified theories from heterotic string compactification on a $Z_{6}$ orbifold, Nucl. Phys. B 704 (2005) 3 [hep-ph/0409098] [INSPIRE].

[16] W. Buchmüller, K. Hamaguchi, O. Lebedev and M. Ratz, Supersymmetric Standard Model from the heterotic string, Phys. Rev. Lett. 96 (2006) 121602 [hep-ph/0511035] [INSPIRE].

[17] W. Buchmüller, K. Hamaguchi, O. Lebedev and M. Ratz, Supersymmetric Standard Model from the heterotic string (II), Nucl. Phys. B 785 (2007) 149 [hep-th/0606187] [INSPIRE].

[18] J.E. Kim and B. Kyae, Flipped SU(5) from $Z_{12-I}$ orbifold with Wilson line, Nucl. Phys. B 770 (2007) 47 [hep-th/0608086] [INSPIRE].

[19] O. Lebedev et al., A mini-landscape of exact MSSM spectra in heterotic orbifolds, Phys. Lett. B 645 (2007) 88 [hep-th/0611095] [INSPIRE]. 
[20] J.E. Kim, J.-H. Kim and B. Kyae, Superstring Standard Model from $Z_{12-I}$ orbifold compactification with and without exotics and effective R-parity, JHEP 06 (2007) 034 [hep-ph/0702278] [INSPIRE].

[21] O. Lebedev et al., The heterotic road to the MSSM with R parity, Phys. Rev. D 77 (2008) 046013 [arXiv: 0708.2691] [inSPIRE].

[22] O. Lebedev, H.P. Nilles, S. Ramos-Sánchez, M. Ratz and P.K.S. Vaudrevange, Heterotic mini-landscape. (II). Completing the search for MSSM vacua in a $Z_{6}$ orbifold, Phys. Lett. B 668 (2008) 331 [arXiv:0807.4384] [INSPIRE].

[23] M. Blaszczyk, S. Groot Nibbelink, M. Ratz, F. Ruehle, M. Trapletti and P.K.S. Vaudrevange, A $Z_{2} \times Z_{2}$ Standard Model, Phys. Lett. B 683 (2010) 340 [arXiv:0911.4905] [INSPIRE].

[24] D.K. Mayorga Pena, H.P. Nilles and P.-K. Oehlmann, A zip-code for quarks, leptons and Higgs bosons, JHEP 12 (2012) 024 [arXiv:1209.6041] [INSPIRE].

[25] S. Groot Nibbelink and O. Loukas, MSSM-like models on $Z_{8}$ toroidal orbifolds, JHEP 12 (2013) 044 [arXiv: 1308.5145] [INSPIRE].

[26] B. Carballo-Pérez, E. Peinado and S. Ramos-Sánchez, $\Delta(54)$ flavor phenomenology and strings, JHEP 12 (2016) 131 [arXiv:1607.06812] [INSPIRE].

[27] V. Braun, Y.-H. He, B.A. Ovrut and T. Pantev, A heterotic Standard Model, Phys. Lett. B 618 (2005) 252 [hep-th/0501070] [INSPIRE].

[28] V. Braun, Y.-H. He, B.A. Ovrut and T. Pantev, A Standard Model from the $E_{8} \times E_{8}$ heterotic superstring, JHEP 06 (2005) 039 [hep-th/0502155] [INSPIRE].

[29] R. Blumenhagen, G. Honecker and T. Weigand, Loop-corrected compactifications of the heterotic string with line bundles, JHEP 06 (2005) 020 [hep-th/0504232] [INSPIRE].

[30] L.B. Anderson, J. Gray, A. Lukas and E. Palti, Heterotic line bundle Standard Models, JHEP 06 (2012) 113 [arXiv: 1202.1757] [INSPIRE].

[31] L.B. Anderson, A. Constantin, J. Gray, A. Lukas and E. Palti, A comprehensive scan for heterotic SU(5) GUT models, JHEP 01 (2014) 047 [arXiv:1307.4787] [INSPIRE].

[32] S. Groot Nibbelink, O. Loukas and F. Ruehle, (MS)SM-like models on smooth Calabi-Yau manifolds from all three heterotic string theories, Fortsch. Phys. 63 (2015) 609 [arXiv: 1507.07559] [INSPIRE].

[33] W. Buchmüller, K. Hamaguchi, O. Lebedev and M. Ratz, Local grand unification, in $C P$ violation and the flavour puzzle: symposium in honour of Gustavo C. Branco. GustavoFest 2005, Lisbon Portugal, July 2005, pg. 143 [hep-ph/0512326] [INSPIRE].

[34] T. Kobayashi, H.P. Nilles, F. Ploger, S. Raby and M. Ratz, Stringy origin of non-Abelian discrete flavor symmetries, Nucl. Phys. B 768 (2007) 135 [hep-ph/0611020] [INSPIRE].

[35] H.P. Nilles and P.K.S. Vaudrevange, Geography of fields in extra dimensions: string theory lessons for particle physics, Mod. Phys. Lett. A 30 (2015) 1530008 [arXiv:1403.1597] [INSPIRE].

[36] M.T. Mueller and E. Witten, Twisting toroidally compactified heterotic strings with enlarged symmetry groups, Phys. Lett. B 182 (1986) 28 [INSPIRE].

[37] T.H. Buscher, A symmetry of the string background field equations, Phys. Lett. B 194 (1987) 59 [INSPIRE]. 
[38] A.A. Tseytlin, Duality symmetric formulation of string world sheet dynamics, Phys. Lett. B 242 (1990) 163 [INSPIRE].

[39] W. Siegel, Superspace duality in low-energy superstrings, Phys. Rev. D 48 (1993) 2826 [hep-th/9305073] [INSPIRE].

[40] K.S. Narain, M.H. Sarmadi and C. Vafa, Asymmetric orbifolds, Nucl. Phys. B 288 (1987) 551 [INSPIRE].

[41] S. Hellerman, J. McGreevy and B. Williams, Geometric constructions of nongeometric string theories, JHEP 01 (2004) 024 [hep-th/0208174] [INSPIRE].

[42] A. Dabholkar and C. Hull, Duality twists, orbifolds and fluxes, JHEP 09 (2003) 054 [hep-th/0210209] [INSPIRE].

[43] J. Shelton, W. Taylor and B. Wecht, Nongeometric flux compactifications, JHEP 10 (2005) 085 [hep-th/0508133] [INSPIRE].

[44] C.M. Hull, A geometry for non-geometric string backgrounds, JHEP 10 (2005) 065 [hep-th/0406102] [INSPIRE].

[45] C.M. Hull, Doubled geometry and T-folds, JHEP 07 (2007) 080 [hep-th/0605149] [INSPIRE].

[46] C. Hull and B. Zwiebach, Double field theory, JHEP 09 (2009) 099 [arXiv:0904.4664] [INSPIRE].

[47] O. Hohm, C. Hull and B. Zwiebach, Background independent action for double field theory, JHEP 07 (2010) 016 [arXiv: 1003.5027] [INSPIRE].

[48] G. Aldazabal, D. Marqués and C. Núñez, Double field theory: a pedagogical review, Class. Quant. Grav. 30 (2013) 163001 [arXiv:1305.1907] [INSPIRE].

[49] D. Lüst, T-duality and closed string non-commutative (doubled) geometry, JHEP 12 (2010) 084 [arXiv: 1010.1361] [inSPIRE].

[50] C. Condeescu, I. Florakis and D. Lüst, Asymmetric orbifolds, non-geometric fluxes and non-commutativity in closed string theory, JHEP 04 (2012) 121 [arXiv:1202.6366] [INSPIRE].

[51] J.A. Harvey, G.W. Moore and C. Vafa, Quasicrystalline compactification, Nucl. Phys. B 304 (1988) 269 [INSPIRE].

[52] L.E. Ibáñez, J. Mas, H.-P. Nilles and F. Quevedo, Heterotic strings in symmetric and asymmetric orbifold backgrounds, Nucl. Phys. B 301 (1988) 157 [INSPIRE].

[53] K.S. Narain, M.H. Sarmadi and C. Vafa, Asymmetric orbifolds: path integral and operator formulations, Nucl. Phys. B 356 (1991) 163 [INSPIRE].

[54] Y. Imamura, M. Sakamoto, T. Sasada and M. Tabuse, Symmetries between untwisted and twisted strings on asymmetric orbifolds, Nucl. Phys. B 390 (1993) 291 [hep-th/9206042] [INSPIRE].

[55] Y. Imamura, M. Sakamoto, T. Sasada and M. Tabuse, String theories on the asymmetric orbifolds with twist-untwist intertwining currents, Prog. Theor. Phys. Suppl. 110 (1992) 261 [hep-th/9202009] [INSPIRE].

[56] T. Sasada, Fermion currents on asymmetric orbifolds, Phys. Lett. B 343 (1995) 128 [hep-th/9312066] [INSPIRE]. 
[57] T. Sasada, Space-time supersymmetry in asymmetric orbifold models, hep-th/9403037 [INSPIRE].

[58] J. Erler, Asymmetric orbifolds and higher level models, Nucl. Phys. B 475 (1996) 597 [hep-th/9602032] [INSPIRE].

[59] K. Aoki, E. D'Hoker and D.H. Phong, On the construction of asymmetric orbifold models, Nucl. Phys. B 695 (2004) 132 [hep-th/0402134] [INSPIRE].

[60] H.S. Tan, T-duality twists and asymmetric orbifolds, JHEP 11 (2015) 141 [arXiv: 1508.04807] [INSPIRE].

[61] Y. Satoh and Y. Sugawara, Lie algebra lattices and strings on T-folds, JHEP 02 (2017) 024 [arXiv: 1611.08076] [INSPIRE].

[62] T.R. Taylor, Model building on asymmetric $Z_{3}$ orbifolds: nonsupersymmetric models, Nucl. Phys. B 303 (1988) 543 [INSPIRE].

[63] Y. Satoh, Y. Sugawara and T. Wada, Non-supersymmetric asymmetric orbifolds with vanishing cosmological constant, JHEP 02 (2016) 184 [arXiv:1512.05155] [INSPIRE].

[64] Y. Sugawara and T. Wada, More on non-supersymmetric asymmetric orbifolds with vanishing cosmological constant, JHEP 08 (2016) 028 [arXiv: 1605.07021] [INSPIRE].

[65] H. Kawai, D.C. Lewellen and S.-H. Henry Tye, Construction of fermionic string models in four-dimensions, Nucl. Phys. B 288 (1987) 1 [INSPIRE].

[66] I. Antoniadis, C.P. Bachas and C. Kounnas, Four-dimensional superstrings, Nucl. Phys. B 289 (1987) 87 [INSPIRE].

[67] P. Athanasopoulos, A.E. Faraggi, S. Groot Nibbelink and V.M. Mehta, Heterotic free fermionic and symmetric toroidal orbifold models, JHEP 04 (2016) 038 [arXiv: 1602.03082] [INSPIRE].

[68] A.E. Faraggi, D.V. Nanopoulos and K.-J. Yuan, A standard like model in the $4 D$ free fermionic string formulation, Nucl. Phys. B 335 (1990) 347 [INSPIRE].

[69] G.B. Cleaver, A.E. Faraggi and D.V. Nanopoulos, String derived MSSM and M-theory unification, Phys. Lett. B 455 (1999) 135 [hep-ph/9811427] [INSPIRE].

[70] A.E. Faraggi, A new standard-like model in the four-dimensional free fermionic string formulation, Phys. Lett. B 278 (1992) 131 [INSPIRE].

[71] A.E. Faraggi, Construction of realistic standard-like models in the free fermionic superstring formulation, Nucl. Phys. B 387 (1992) 239 [hep-th/9208024] [INSPIRE].

[72] F. Beye, T. Kobayashi and S. Kuwakino, Gauge symmetries in heterotic asymmetric orbifolds, Nucl. Phys. B 875 (2013) 599 [arXiv:1304.5621] [INSPIRE].

[73] F. Beye, T. Kobayashi and S. Kuwakino, Three-generation asymmetric orbifold models from heterotic string theory, JHEP 01 (2014) 013 [arXiv:1311.4687] [INSPIRE].

[74] F. Beye, T. Kobayashi and S. Kuwakino, Dilaton stabilization in three-generation heterotic string model, Phys. Lett. B 760 (2016) 63 [arXiv:1603.08313] [INSPIRE].

[75] W. Lerche, D. Lüst and A.N. Schellekens, Chiral four-dimensional heterotic strings from selfdual lattices, Nucl. Phys. B 287 (1987) 477 [INSPIRE].

[76] K.S. Narain, New heterotic string theories in uncompactified dimensions $<10$, Phys. Lett. B 169 (1986) 41 [INSPIRE]. 
[77] D. Gepner, Space-time supersymmetry in compactified string theory and superconformal models, Nucl. Phys. B 296 (1988) 757 [InSPIRE].

[78] D. Gepner, Exactly solvable string compactifications on manifolds of $\mathrm{SU}(N)$ holonomy, Phys. Lett. B 199 (1987) 380 [INSPIRE].

[79] B. Gato-Rivera and A.N. Schellekens, Asymmetric Gepner models: revisited, Nucl. Phys. B 841 (2010) 100 [arXiv: 1003.6075] [INSPIRE].

[80] B. Gato-Rivera and A.N. Schellekens, Asymmetric Gepner models II: heterotic weight lifting, Nucl. Phys. B 846 (2011) 429 [arXiv:1009.1320] [INSPIRE].

[81] A.N. Schellekens, Big numbers in string theory, arXiv:1601.02462 [INSPIRE].

[82] D. Israël and V. Thiéry, Asymmetric Gepner models in type-II, JHEP 02 (2014) 011 [arXiv: 1310.4116] [INSPIRE].

[83] D. Israël, Nongeometric Calabi-Yau compactifications and fractional mirror symmetry, Phys. Rev. D 91 (2015) 066005 [Erratum ibid. D 91 (2015) 129902] [arXiv:1503.01552] [INSPIRE].

[84] R. Blumenhagen, M. Fuchs and E. Plauschinn, The asymmetric CFT landscape in D=4,6,8 with extended supersymmetry, Fortsch. Phys. 65 (2017) 1700006 [arXiv:1611.04617] [INSPIRE].

[85] R. Blumenhagen, A. Deser, E. Plauschinn, F. Rennecke and C. Schmid, The intriguing structure of non-geometric frames in string theory, Fortsch. Phys. 61 (2013) 893 [arXiv: 1304.2784] [INSPIRE].

[86] R. Blumenhagen and R. Sun, T-duality, non-geometry and Lie algebroids in heterotic double field theory, JHEP 02 (2015) 097 [arXiv: 1411.3167] [inSPIRE].

[87] R. Vaidyanathaswamy, Integer-roots of the unit matrix, J. Lond. Math. Soc. 3 (1928) 121.

[88] J. Polchinski, String theory. Vol. 2: superstring theory and beyond, Cambridge University Press, Cambridge U.K., (2007) [INSPIRE].

[89] S. Bittanti, A.J. Laub and J.C. Willems, The Riccati equation, Springer Science \& Business Media, Germany, (2012).

[90] M. Cvetič, J. Louis and B.A. Ovrut, A string calculation of the Kähler potentials for moduli of $Z_{N}$ orbifolds, Phys. Lett. B 206 (1988) 227 [InSPIRE].

[91] A. Dabholkar and J.A. Harvey, String islands, JHEP 02 (1999) 006 [hep-th/9809122] [INSPIRE]. 